\title{
Falling flavors in AdS/CFT
}

\author{
Paul McGuirk \\ Laboratory for Elementary-Particle Physics, Cornell University, Ithaca, New York, 14853, USA \\ mcguirk@cornell.edu
}

\begin{abstract}
We consider the behavior of D7 probes of supersymmetric warped geometries that are perturbed by the presence of $\overline{\mathrm{D} 3}$-branes. Such constructions are the gravitational duals of non-supersymmetric states in supersymmetric flavored gauge theories. Although the D7s we consider do not feel a force from either D3s or $\overline{\mathrm{D} 3}$ s alone, when both types of 3-branes are present the D7s deform and fall a small distance toward the 3-branes. We perform our analysis in $A d S^{5} \times S^{5}$ and $A d S^{5} \times T^{1,1}$ and find qualitatively similar behavior in each case. We then extend our consideration to the approximately conical region of the Klebanov-Strassler solution and find that the effect is parametrically larger than in the $A d S^{5} \times X^{5}$ examples. Additionally, we discuss how these behaviors are modified by the presence of other flavors by considering the smeared backreaction of such flavor branes in $A d S^{5} \times X^{5}$. Finally, we touch upon some of the implications that our results may have for model building and argue that the deformation of the worldvolume results in $\mathcal{O}(1)$ corrections to soft terms in the low-energy description.
\end{abstract}




\section{Contents}

1 Introduction $\quad 1$

2 Preliminaries and summary of results 3

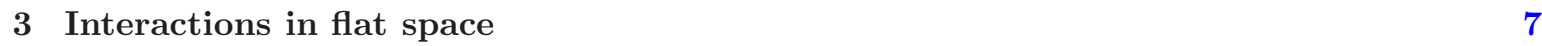

3.1 Bending of probe D7s

3.2 Backreacting D7s 14

$\begin{array}{lll}3.3 & \text { Forces between flavors } & 17\end{array}$

4 Interactions on the conifold $\quad 21$

4.1 Kuperstein probes 23

4.2 Backreacting Kupersteins 26

$5 \quad$ D7s and anti-D3s in Klebanov-Tseytlin 28

6 Model building with falling flavors $\quad 35$

$\begin{array}{lll}7 & \text { Concluding remarks } & 38\end{array}$

$\begin{array}{ll}\text { A Type-IIB supergravity } & 39\end{array}$

$\begin{array}{ll}\text { B Backreacting 3-branes on Calabi-Yau cones } & 40\end{array}$

$\begin{array}{ll}\text { C Backreaction of smeared D7s } & 41\end{array}$

C.1 D7s and 3-branes in flat space $\quad 41$

C.2 Kuperstein branes on the conifold $\quad 46$

\section{Introduction}

Warped geometries continue to play a prominent role in string theory. The furthest-reaching application of such constructions is the gauge/gravity correspondence [1-4] which provides for a deeper understanding of strongly coupled gauge theories and the nature of quantum gravity. As a consequence of this duality, warped geometries also provide a fruitful corner of the landscape in which to search for controlled and phenomenologically promising vacua. For example, the large redshift resulting from strong warping can naturally accommodate an exponentially large separation of mass scales [5-7], just as dimensional transmutation produces such hierarchies in gauge theories. Additionally, warping brings to string constructions the powerful tools of effective field theories in which unknown bulk effects can be parametrized by Wilson coefficients and systematically incorporated (see, e.g., [8, 9]).

However, in order to reach the goal of phenomenological viability, the string modes that give rise to visible-sector fields must also be included. In the widely explored GKP-like warped constructions of the type-IIB superstring [7], such modes are the open-string fluctuations of either (fractional or whole) D3-branes or D7-branes ${ }^{1}$. Although D3-branes provide a rich framework for model building and have

\footnotetext{
${ }^{1}$ In more general F-theoretic constructions, we could of course consider other types of 7 -branes or even in principle other types of fractional 3-branes.
} 
the extra benefit of being very local, there are many phenomenological advantages to considering scenarios in which the visible sector arises from D7-branes. For example, one of the successes of the so-called "bulk" Randall-Sundrum (RS) scenarios is that a hierarchical structure of Yukawa couplings can be arranged by $\mathcal{O}(1)$ tunings of the $5 \mathrm{~d}$ masses of fermions propagating in a warped geometry [10]; such $5 \mathrm{~d}$ masses control the internal profiles of the fermions and hence their overlap with scalar fields. In a stringy realization of an RS-like scenario, such modes correspond to the excitations of D7-branes that extend some distance into the warped geometry. Chiral modes in particular are realized on the intersections of magnetized D7-branes and hence the string theory "lift" of $5 \mathrm{~d}$ mass is, at least morally if not literally, the position of D7-branes that extend into the warped region ${ }^{2}$. Hence, it is expected that $\mathcal{O}(1)$ adjustments of the positions of D7-branes can produce hierarchical Yukawa textures. The addition of such D7-branes to a warped geometry has the well-known interpretation of adding a global flavor group to the dual gauge theory [13] and hence the branes are often called flavor branes.

A further phenomenological constraint is the absence of supersymmetry, at least at long wavelengths. Unfortunately, the construction of non-supersymmetric configurations in string theory is difficult to achieve. In additional to the practical difficulties associated with solving second-order as opposed to first-order differential equations, the stability of non-supersymmetric geometries is often an issue ${ }^{3}$. One method of breaking supersymmetry in a relatively controlled way is the addition of anti-branes to a warped geometry. For example, the branes and fluxes that build a GKP-like warped region carry positive D3-brane charge and so an $\overline{\mathrm{D} 3}$-brane, which carries negative D3-brane charge and hence breaks the supersymmetry preserved by the geometry, will be naturally attracted to the point of strongest warping and largest redshift. The warping then serves to restrict the influence of the $\overline{\mathrm{D} 3}$-brane to the strongly warped region so that remainder of the geometry is approximately supersymmetric. Furthermore, in examples such as the Klebanov-Strassler (KS) solution [16] in which the warping is not sourced by integer D3-branes, the $\overline{\mathrm{D} 3}$-branes will be perturbatively stable so long as the number of anti-branes is small with respect to the number of flux quanta that support the geometry [17]. Due to the localized nature of the $\overline{\mathrm{D} 3}$-branes, their backreaction falls off sufficiently fast with the distance from the branes that the configuration is dual to a particular non-supersymmetric state in a supersymmetric theory [18], a fact that has been used to explore gravitational duals of gauge mediation [19-22] (see also [23-28] $)^{4}$.

Warping, D7-branes, and $\overline{\mathrm{D} 3}$-branes also play an important role in the KKLT scenario for achieving metastable de Sitter spaces [41] and the related framework for inflation [42]. In each of these cases, gaugino condensation on D7-branes provides a mechanism for the stabilization of the Kähler structure of the internal space while $\overline{\mathrm{D} 3}$-branes are central in the construction of the uplift and inflationary potentials.

Given the many examples in which D7-branes and $\overline{\mathrm{D} 3}$-branes appear together in warped geometries with D3 charge, it is important to understand how these objects interact with each other. As is well

\footnotetext{
${ }^{2}$ The story will be a little more complicated in detail. For example, the localization of fermionic modes also depends on the magnetic flux as in the D-brane examples of $[11,12]$.

${ }^{3}$ For some recent examples of non-supersymmetric warped geometries, see [14, 15].

${ }^{4}$ Note that as a consequence of the non-linear behavior of the supergravity equations of motion, the backreaction of $\overline{\mathrm{D} 3}$-branes in a GKP construction results in singular 3-form flux despite the fact that such 3-branes do not carry any NS-NS or R-R 2-form charge [29-36] (see also [37-40] for a T-dual case). However, as this behavior is directly linked to the brane singularities that are expected to be resolved in the full string theory, in our opinion the singularities are likely an artifact of the supergravity approximation which breaks down near the position of the branes. As the background at large distances is essentially determined by the net charge and mass of the solution, if it is the case that there is some stringy resolution to the singularity, then the effect of such resolution on D7 probes is probably subleading as long as the D7s are sufficiently far from the supergravity singularity.
} 
known, D7s do not experience a net force from "parallel" D3-branes. Furthermore, since a D7 carries no D3-brane charge (unless such a charge is induced by generalized worldvolume flux or curvature) and hence cannot distinguish a D3-brane from an D3-brane, D7s do not experience a force from parallel $\overline{\mathrm{D}} \mathrm{s}$ either. However, due to the non-linear nature of supergravity, when both D3s and $\overline{\mathrm{D} 3} \mathrm{~s}$ are present there is no guarantee that the D7-branes will experience no force. One may further expect that in the presence of a non-supersymmetric background, parallel D7-branes may exert a force on each other, despite the fact that when only D3-branes or $\overline{\mathrm{D} 3}$-branes were present there was an exact cancellation between the NS-NS attraction and the R-R repulsion.

It is precisely such interactions that we explore in this work. In section 2 we argue generally from the equations of motion that D7-branes which feel no force in the presence of only one type of D3-brane will bend in the presence of both. As much of the paper contains lengthy calculations, section 2 also contains a summary of our results. In section 3 we perform a more quantitative analysis by considering D7-probes of $A d S^{5} \times S^{5}$ perturbed by a small number of $\overline{\mathrm{D} 3}$-branes and find that indeed the D7-branes tend to fall a little toward the non-BPS stack of 3-branes. We additionally show how this effect can be modified by the presence of other D7-branes by considering their backreaction as well. In section 4, we perform the analogous analysis for Kuperstein probes of $A d S^{5} \times T^{1,1}$ and find that, due to common conical structure of the geometries, the qualitative behavior is the same. In section 5 we consider D7-probes of the Klebanov-Tseytlin geometry perturbed by $\overline{\mathrm{D} 3}$-branes. In this case, due to the presence of the background 3-form flux, the probe D7s experience a parametrically stronger attraction to the non-supersymmetric source of warping. In section 6 we briefly and qualitatively discuss some aspects of the impact the deformations will have on model building in warped compacitifcations. Some concluding remarks are made in section 7 . Our conventions and some technical details regarding brane backreaction are relegated to appendices.

\section{Preliminaries and summary of results}

As is very well known, D3-branes and D7-branes are, in many situations, mutually BPS objects and hence there is no net force between them (see, e.g. [43]). As a simple example, consider a stack of $N$ D3-branes in $R^{9,1}$. A D7-brane that sits parallel to the D3s and fills four orthogonal directions will preserve half of the supersymmetry preserved by the D3s. The absence of a force can be seen by considering the D7 as a probe of the D3 background. The backreaction of the D3-branes takes the familiar form ${ }^{5}$

$$
\mathrm{d} s_{10}^{2}=\mathrm{e}^{2 A} \eta_{\mu \nu} \mathrm{d} x^{\mu} \mathrm{d} x^{\nu}+\mathrm{e}^{-2 A} \delta_{m n} \mathrm{~d} y^{m} \mathrm{~d} y^{n},
$$

in which the warp factor $A$ is a function of the coordinates $y^{m}$ that are transverse to the D3s. In addition, D3s are both electric and magnetic sources for $C_{4}$ and hence

$$
F_{5}=(1+\hat{*}) g_{\mathrm{s}}^{-1} \mathrm{de}^{4 A} \wedge \operatorname{dvol}_{R^{3,1}},
$$

in which $\operatorname{vol}_{R^{3,1}}=\mathrm{d} x^{0} \wedge \mathrm{d} x^{1} \wedge \mathrm{d} x^{2} \wedge \mathrm{d} x^{3}$ is the volume element for $R^{3,1}$. In the absence of brane flux, the D7 does not couple to $C_{4}$ and hence the potential does not contribute to the D7 CS action (A.6). Similarly, in the absence of fluctuations of the D7, the D3s do not contribute to the DBI action for the D7; the metric on the D7 worldvolume takes the form

$$
\mathrm{d} s_{8}^{2}=\hat{g}_{\alpha \beta} \mathrm{d} x^{\alpha} \mathrm{d} x^{\beta}=\mathrm{e}^{2 A} \eta_{\mu \nu} \mathrm{d} x^{\mu} \mathrm{d} x^{\nu}+\mathrm{e}^{-2 A} \delta_{a b} \mathrm{~d} y^{a} \mathrm{~d} y^{b},
$$

\footnotetext{
${ }^{5}$ Our conventions are presented in appendix A.
} 
and hence the volume element for the D7 worldvolume, $\operatorname{dvol}_{\mathcal{W}^{8}}=\mathrm{d}^{8} x \sqrt{-\operatorname{det}\left(\hat{g}_{\alpha \beta}\right)}$, is independent of the warp factor. Since neither the warp factor nor $C_{4}$ contribute to the non-derivative parts of the D7 action, the D7 feels no force from the D3.

The absence of a force can also be seen from the D3 perspective. Instead of $C_{4}$, the D7 sources the axiodilaton to which a D3-brane does not couple, while the backreaction on the metric is "limited" to a deficit angle in the plane transverse to the D7 and hence is not felt by the D3.

From either point of view, the Ramond-Ramond potential from the other brane did not contribute to probe brane action and hence the absence of a force will result if we replace all of the D3-branes by $\overline{\mathrm{D} 3} \mathrm{~s}$. Equivalently, of the sixteen supercharges preserved by the D7, eight are also preserved by D3s, while the other eight are preserved by $\overline{\mathrm{D} 3} \mathrm{~s}$ and so D7s cannot, by themselves, distinguish the sign of the charge of the 3-branes. In a linear theory, this would imply that a D7-brane in the presence of both types of 3-branes would feel no force. However, supergravity is a non-linear theory and we will find that there is an interaction when both 3-branes are present. Indeed in the presence of both D3s and $\overline{\mathrm{D} 3} \mathrm{~s}$, no supercharges are preserved and hence there is no BPS bound to protect against the development of a force.

To see this more explicitly, we consider a more general warped ansatz

$$
\mathrm{d} s_{10}^{2}=\mathrm{e}^{2 A(y)} \eta_{\mu \nu} \mathrm{d} x^{\mu} \mathrm{d} x^{\nu}+\mathrm{e}^{-2 A(y)} g_{m n} \mathrm{~d} y^{m} \mathrm{~d} y^{n},
$$

in which $\eta_{\mu \nu}$ is the Minkowski metric on $R^{3,1}$ and $g_{m n}$ is the metric on a space $Y^{6}$. The geometry is again supported by various fluxes

$$
F_{5}=(1+\hat{*}) g_{\mathrm{s}}^{-1} \mathrm{~d} \omega(y) \wedge \operatorname{dvol}_{R^{3,1}}, \quad \iota_{\partial_{\mu}} G_{3}=0,
$$

in which $\iota$ is the interior product. We orient the 3-branes along the Minkowski directions and take any D7-branes to fill the Minkowski directions and wrap 4-cycles $\Sigma_{i}^{4}$. It is useful to define the quantities [7]

$$
\Phi_{ \pm}=\mathrm{e}^{4 A} \pm \omega, \quad G_{ \pm}=(\mathrm{i} \pm *) G_{3}, \quad \Lambda=\Phi_{+} G_{-}+\Phi_{-} G_{+} .
$$

Then, keeping in mind that D7s are magnetic sources for $C_{0}$, the equations of motion following from the bosonic action and the self-duality condition for $F_{5}$ are

$$
\begin{aligned}
0= & \nabla^{2} \Phi_{ \pm}-\frac{2}{\Phi_{+}+\Phi_{-}}\left(\partial \Phi_{ \pm}\right)^{2}-\frac{g_{\mathrm{s}}\left(\Phi_{+}+\Phi_{-}\right)^{2}}{16 \operatorname{Im} \tau}\left|G_{ \pm}\right|^{2} \\
& -\frac{\left(\Phi_{+}+\Phi_{-}\right)^{2}}{2}(2 \pi)^{4} \alpha^{2} g_{\mathrm{s}} \sum_{\mathrm{D} 3^{ \pm}} \frac{\delta^{6}\left(y-y_{a}\right)}{\sqrt{g}}, \\
0= & R_{m p}-\frac{2}{\left(\Phi_{+}+\Phi_{-}\right)^{2}} \partial_{(m} \Phi_{+} \partial_{p)} \Phi_{-}-\frac{1}{2(\operatorname{Im} \tau)^{2}} \partial_{(m} \tau \partial_{p)} \bar{\tau} \\
& +\frac{g_{\mathrm{s}}\left(\Phi_{+}+\Phi_{-}\right)}{16 \cdot 2 ! \operatorname{Im} \tau}\left[G_{+(m}{ }^{n_{1} n_{2}} \bar{G}_{-p) n_{1} n_{2}}+G_{-(m}{ }^{n_{1} n_{2}} \bar{G}_{+p) n_{1} n_{2}}\right] \\
& -\frac{\mathrm{e}^{\phi}}{2} \sum_{\mathrm{D} 7} \frac{1}{\sqrt{\Omega_{i}^{2}}}\left(\Omega_{i}\right)_{m n}\left(\Omega_{i}\right)_{p}^{n}, \\
0= & \nabla^{2} \tau+\frac{\mathrm{i}}{\operatorname{Im} \tau}(\partial \tau)^{2}+\frac{\mathrm{i} g_{\mathrm{s}}\left(\Phi_{+}+\Phi_{-}\right)}{8} G_{+} \cdot G_{-}+\mathrm{i} \sum_{\mathrm{D} 7} \sqrt{\Omega_{i}^{2}}, \\
0= & \mathrm{d} F_{1}+\sum_{\mathrm{D} 7} \Omega_{i},
\end{aligned}
$$




$$
\begin{aligned}
& 0=\mathrm{d} \Lambda+\frac{\mathrm{i}}{2 \operatorname{Im} \tau} \mathrm{d} \tau \wedge(\Lambda+\bar{\Lambda}) \\
& 0=\mathrm{d}\left(G_{+}-G_{-}\right)+\frac{\mathrm{i}}{2 \operatorname{Im} \tau} \mathrm{d} \tau \wedge\left(G_{+}+\bar{G}_{+}-G_{-}-\bar{G}_{-}\right),
\end{aligned}
$$

in which $\Omega_{i}$ are the Poincaré duals in $Y^{6}$ of the 4-cycles wrapped by the D7s (see, e.g. [44]), the D3-branes and $\overline{\mathrm{D} 3}$-branes (denoted also D3 ${ }^{+}$and D3 ${ }^{-}$respectively) are localized at points in $Y^{6}$, and $R_{m p}$ and related quantities are built from the unwarped $6 \mathrm{~d}$ metric $g_{m p}$. In writing these equations of motion, we have neglected the presence of worldvolume flux on any of the branes.

We first consider the case in which $G_{3}$ vanishes and the D7-brane contribution to the equations of motion can be neglected (i.e. treating the D7s as probe branes). In such a setup, when only D3 or only $\overline{\mathrm{D} 3} \mathrm{~s}$ are present (and hence only one of $\Phi_{+}$or $\Phi_{-}$is non-vanishing), the internal ${ }^{6}$ metric is unchanged by the presence of 3-branes and correspondingly a probe D7 will not feel their influence. However, when both types of branes are present, $\Phi_{+}$and $\Phi_{-}$together source $g_{m p}$ so that the internal metric can no longer be Ricci flat. Hence, although the warp factor cancels out of the DBI action for a probe D7-brane, the non-trivial metric will generically cause the D7 to bend as it extremizes its volume.

We can also easily see the non-trivial interactions from the point of view of probe 3 -branes. We consider for example a background with $N$ D3-branes and $N_{f}$ D7-branes. The equations of motion will then imply that $\Phi_{+}^{-1}$ is harmonic. However, since the D7s backreact on the internal metric forcing it to no longer be Ricci flat, $\Phi_{+}^{-1}$ will take on a different form than it would in the absence of D7 branes. Since $\Phi_{+}$is precisely the potential felt by an $\overline{\mathrm{D} 3}$ brane, such a brane will feel a potential with terms proportional to $N N_{f}$ in addition to those depending on just $N$. Similar arguments could be for D3-probes.

In the following sections, we will work out some of the details of these interactions in particular examples. However, since our presentation is somewhat explicit, we will briefly summarize our results here. We work in strongly warped geometries so that the backreaction of the D7s is under some degree of control. This is easiest to understand in the dual gauge theory as the addition of $N_{f}$ D7-branes to a warped geometry of the type considered here corresponds to the addition of a $\mathrm{U}\left(N_{f}\right)$ flavor group [13]. Such flavors can be treated as non-dynamical objects in the 't Hooft limit in which we take the number of colors $N \rightarrow \infty$ while letting $\frac{N_{f}}{N} \rightarrow 0$ (see, for example, the discussion in [45]), and hence their influence on the gauge theory can be largely neglected for a wide range of energies. Correspondingly, many components of the backreacted geometry scale as $\frac{N_{f}}{N}$ and hence for $N_{f} \ll N$, the backreaction can be largely neglected, at least for large ranges of $r$.

The simplest examples of such strongly warped geometries the near-brane geometries of $N$ D3s sitting at the apex of a Calabi-Yau cone ${ }^{7}$. The near-brane geometry is then $A d S^{5} \times X^{5}$

$$
\mathrm{d} s_{10}^{2}=\frac{r^{2}}{L^{2}} \eta_{\mu \nu} \mathrm{d} x^{\mu} \mathrm{d} x^{\nu}+\frac{L^{2}}{r^{2}} \mathrm{~d} r^{2}+L^{2} \mathrm{~d} s_{X^{5}}^{2}
$$

\footnotetext{
${ }^{6}$ Note that since we are not considering explicit compactifications, the term "internal" is inaccurate here. We use the term to describe directions transverse to the Minkowski directions as these are the ones that would eventually become the coordinates on a compact space.

${ }^{7}$ Note that in taking the near-brane limit of the D3 background, we remove the explicit D3 sources from and dissolve the flux into the geometry. Thus our analysis will not completely capture the physics of the interactions between the D7s and the different D3-branes (except near the horizon). Nevertheless, the analysis should capture the qualitative behavior, just as the attraction of probe $\overline{\mathrm{D} 3}$ brane to the origin of $A d S^{5}$ reflects the attraction of $\overline{\mathrm{D} 3}$ branes to D3s
} 
in which $X^{5}$ is the Sasaki-Einstein base of the Calabi-Yau cone and $L^{4} \sim g_{\mathrm{s}} N \alpha^{2}$ sets the radius of both the $A d S^{5}$ and $X^{5}$ factors. A D7 that is holomorphically embedded into the Calabi-Yau preserves some of the supersymmetry of the geometry and hence does not experience a force. On the other hand, the backreaction of $p \ll N \overline{\mathrm{D} 3}$ s (which was found in [18] and is reviewed in appendix B) includes a squashing of the metric so that it is no longer Ricci flat and hence the shape of the volume-minimizing configuration must change. By analyzing the probe D7 action, we show that when the Sasaki-Einstein base is $S^{5}$ or $T^{1,1}$ the $\mathrm{D} 7$ brane deforms so that the minimum radius reached by the D7 decreases (see (3.33) and (4.24))

$$
\frac{\Delta r}{r_{\min }} \sim-\frac{p}{N} \frac{L^{8}}{r_{\min }^{8}}
$$

where $r_{\min }$ is the minimum radius obtained by the D7-brane in the supersymmetric case. The details of the shape depend on the initial embedding and which Einstein-Sasaki base space is used. A cartoon of the deformed embedding in the $S^{5}$ case is given in figure 2.

If we treat the $\overline{\mathrm{D} 3} \mathrm{~s}$ as a probe, then the above discussion suggests that the force experienced by the $\overline{\mathrm{D} 3}$ probe will be modified by the backreaction of the D7s. The backreaction of such D7s is difficult to find and to make progress we use the smeared solutions reviewed in [45]. As a consequence of such smearing, the geometry at radii inside the minimum radius of the smeared branes is not modified by the presence of the branes. Hence, to obtain a non-vanishing force, we must place the $\overline{\mathrm{D} 3}$ brane at large radii and so the configuration is not the same as the configuration that lead to (2.9) and we should not expect to reconstruct the precise physics. However the resulting modification of the potential confirms the non-trivial interaction of the branes. In this case, the potential for the canonically normalized field representing the position of the $\overline{\mathrm{D} 3}$ brane takes the same functional form (at least when the D7s are treated as a perturbation) $V(\sigma) \sim \sigma^{4 / 3}$, but the coefficient is reduced by $a \frac{g_{\mathrm{s}} N_{f}}{2 \pi}$ where $a \sim \mathcal{O}(1)$ (see (3.55)). We only explicitly perform this calculation in the $S^{5}$ case, but the same qualitative behavior arises from any Einstein-Sasaki base.

The potential for D3 probes when the backreaction of the D7s and $\overline{\mathrm{D} 3} \mathrm{~s}$ (now returned to small $r$ ) are taken into account takes the schematic form (3.57)

$$
V(\sigma) \sim-\frac{p}{N}\left(1+\frac{g_{\mathrm{s}} N_{f}}{2 \pi}\right) \frac{1}{\sigma^{4 / 3}}-\frac{p^{2}}{N^{2}} \frac{1}{\sigma^{4}},
$$

where we have specialized to the case where the $\overline{\mathrm{D} 3} \mathrm{~s}$ and D7s are treated as comparable perturbations (see appendix C). We again only explicitly considered the $S^{5}$ case, but other bases have the same scaling.

When $\overline{\mathrm{D} 3} \mathrm{~s}$ are present and supersymmetry is broken, the cancellation of NS-NS and R-R forces between two D7-branes should no longer occur. We can confirm this fact by considering a probe D7-brane in the geometry produced by backreacting $\overline{\mathrm{D} 3} \mathrm{~s}$ and other D7s. Indeed, we find that at large distances the backreacting D7s repel the probe D7, which has again been placed at a larger radius. Schematically, (see (3.79) and (4.36))

$$
\frac{\Delta r}{r_{\min }} \sim-\frac{p}{N} \frac{L^{8}}{r_{\min }^{8}}-\frac{p^{2}}{N^{2}} \frac{L^{16}}{r_{\min }^{16}}-\frac{p}{N} \frac{g_{\mathrm{s}} N_{f}}{2 \pi} \frac{L^{8}}{r_{\min }^{8}}\left[1+\log \frac{r_{\min }}{r_{\mathrm{s}}}\right]
$$

in which $r_{\mathrm{s}}$ is an integration constant characterizing the backreaction of the D7s. Again, to find this expression we treat the $\overline{\mathrm{D} 3} \mathrm{~s}$ and D7s as comparable perturbations to the geometry. Because of the logarithmic behavior of the backreaction of the D7s, treating the D7s as a small perturbation is a valid approximation only over a certain range of radii. However, it is interesting to note that naive application of (2.11) for sufficiently small $r_{\text {min }}$ indicates that the backreacting D7s repel the probe D7. 
Finally, we turn to the case of D7 probes in the warped deformed conifold perturbed by $\overline{\mathrm{D} 3}$-branes. The full linearized solution provided by [30-36] is rather involved and we use the solution provided by [18] in which $\overline{\mathrm{D} 3} \mathrm{~s}$ are added to the Klebanov-Tseytlin solution. Because of the increased complexity of the geometry even in this case, we do not attempt to backreact any D7s, but for a probe D7 we again find a tendency for the branes to deform (see (5.33)

$$
\frac{\Delta r}{r_{\min }} \sim-\mathcal{S} \frac{\alpha^{\prime 2}}{r_{\min }^{4}}
$$

in which $\mathcal{S}$ characterizes the perturbation by the $\overline{\mathrm{D} 3} \mathrm{~s}$. The different functional form of this behavior is a consequence of the 3 -form flux present in the geometry.

In all of the above examples we have treated the $\overline{\mathrm{D} 3}$-branes as a perturbation to the geometry. However, since the solution grows with decreasing $r$, perturbation theory breaks down at sufficiently small radii. Hence, our treatment is valid only for sufficiently large $r$. Fortunately, the deformation of the worldvolumes of the D7 is sufficiently small that the D7s, even after bending, do not reach the point where the perturbative treatment breaks down and so (2.9) and (2.12) can be trusted. Similar statements apply when we include the backreaction of D7s, however there is the additional constraint that the geometry becomes singular for large $r$ as well (corresponding to the presence of a Landau pole in a dual theory). For small $g_{\mathrm{s}} N_{f}$, we can easily arrange things so that there is a wide range of initial embeddings such that the perturbative treatment is valid $^{8}$.

\section{Interactions in flat space}

We now return to the flat space case and examine more precisely how a D7-brane will respond to the presence of both types of 3-branes. Keeping in mind the uplift scenario of [41], we will consider the addition of $p$ D3- $\overline{\mathrm{D} 3}$ pairs to $N \gg p$ D3s. When the 3-branes are coincident, we can take the ansatz for the backreaction of the 3-branes to be of the type (2.4) with

$$
g_{m n} \mathrm{~d} y^{m} \mathrm{~d} y^{n}=\mathrm{e}^{2 B}\left(\mathrm{~d} r^{2}+r^{2} \mathrm{~d} s_{S^{5}}^{2}\right),
$$

where $\mathrm{d} s_{S^{5}}^{2}$ is the metric for a unit $S^{5}$, the branes are located at $r=0$, and all fields other than the metric on $S^{5}$ can be taken as functions of $r$ alone. In the more general case of coincident 3-branes stacked at the bottom of a Calabi-Yau cone, the same ansatz applies where $S^{5}$ is replaced by the appropriate Sasaki-Einstein manifold. In this setup, the coincident branes are perturbatively unstable and so the configuration should be viewed as a warm-up for the metastable configuration discussed later.

The solution for any values of $p$ and $N$ were presented in [46] (following [47]). As discussed in the introduction, our interest is in strongly warped geometries. Such a geometry follows from taking the near-brane limit of the 3-brane geometry. The backreaction in this limit and for $p \ll N$ was discussed in [18], which we review and carry out to higher order in $\frac{p}{N}$ in appendix B. The result is

$$
\begin{aligned}
\mathrm{e}^{-4 A} & =\frac{L^{4}}{r^{4}}+\frac{4 p}{5 N} \frac{L^{12}}{r^{12}}+\frac{54 p^{2}}{125 N^{2}} \frac{L^{20}}{r^{20}}, \\
\omega & =\frac{r^{4}}{L^{4}}+\frac{6 p}{5 N} \frac{L^{4}}{r^{4}}-\frac{24 p^{2}}{125 N^{2}} \frac{L^{12}}{r^{12}}, \\
\mathrm{e}^{2 B} & =1-\frac{p}{5 N} \frac{L^{8}}{r^{8}}-\frac{p^{2}}{50 N^{2}} \frac{L^{16}}{r^{16}} .
\end{aligned}
$$

\footnotetext{
${ }^{8}$ Note that although D7s are noncompact and hence extend out to infinite radius, the deviation from the supersymmetric embedding is localized in the region where a perturbative treatment is valid.
} 
$S^{5}$ is an Einstein-Sasaki space and therefore can be written as a U (1) fibration over an EinsteinKähler base, a fact that was exploited in a related context in [44]. Indeed writing

$$
z^{1}=r \cos \frac{\gamma}{2} \cos \frac{\theta}{2} \mathrm{e}^{\mathrm{i}(\psi+\eta / 2+\varphi / 2)}, \quad z^{2}=r \cos \frac{\gamma}{2} \sin \frac{\theta}{2} \mathrm{e}^{\mathrm{i}(\psi+\eta / 2-\varphi / 2)}, \quad z^{3}=r \sin \frac{\gamma}{2} \mathrm{e}^{\mathrm{i} \psi},
$$

in which $r \in[0, \infty), \gamma, \theta \in[0, \pi], \varphi, \psi \in[0,2 \pi)$, and $\eta \in[0,4 \pi)$, the metric for $R^{6}=C^{3}$ takes the form

$$
\mathrm{d} s_{6}^{2}=\mathrm{d} z^{i} \mathrm{~d} \bar{z}^{\bar{i}}=\mathrm{d} r^{2}+r^{2}(\mathrm{~d} \psi+\mathcal{A})^{2}+r^{2} \mathrm{~d} s_{C P^{2}}^{2},
$$

in which

$$
\mathcal{A}=\frac{1}{2} \cos ^{2} \frac{\gamma}{2}(\mathrm{~d} \eta+\cos \theta \mathrm{d} \varphi)
$$

The $4 \mathrm{~d}$ metric

$$
\mathrm{d} s_{C P^{2}}^{2}=\frac{1}{4} \mathrm{~d} \gamma^{2}+\frac{1}{4} \cos ^{2} \frac{\gamma}{2}\left(\mathrm{~d} \theta^{2}+\sin ^{2} \theta \mathrm{d} \varphi^{2}\right)+\frac{1}{4} \cos ^{2} \frac{\gamma}{2} \sin ^{2} \frac{\gamma}{2}(\mathrm{~d} \eta+\cos \theta \mathrm{d} \varphi)^{2},
$$

is the Fubini-Study metric on the Einstein-Kähler space $C P^{2}$. This can be confirmed by considering, for example, the inhomogeneous coordinates $w^{1,2}=\frac{z^{1,2}}{z^{3}}$ on the $z^{3} \neq 0$ patch. Finally, we note that the Kähler form on $C P^{2}$ is given by

$$
\mathcal{J}=-\frac{1}{4} \sin \frac{\gamma}{2} \cos \frac{\gamma}{2} \mathrm{~d} \gamma \wedge(\mathrm{d} \eta+\cos \theta \mathrm{d} \varphi)-\frac{1}{4} \cos ^{2} \frac{\gamma}{2} \sin \theta \mathrm{d} \theta \wedge \mathrm{d} \varphi=\frac{1}{2} \mathrm{~d} \mathcal{A},
$$

and that the Kähler form on $C^{3}$ is

$$
J=r \mathrm{~d} r \wedge(\mathrm{d} \psi+\mathcal{A})+r^{2} \mathcal{J} .
$$

When $p=0$, the geometry is $A d S^{5} \times S^{5}$ which is famously dual to the conformal $\mathcal{N}=4 \mathrm{SU}(N)$ super Yang-Mills theory [1].

\subsection{Bending of probe D7s}

We now introduce a probe D7 into the above geometry. Note that since the backreaction of the $\overline{\mathrm{D} 3}$ grows with decreasing radius, we must have that the probe $\mathrm{D} 7$ does not extend to very small $r$. When $p=0$, the geometry (3.2) is supersymmetric and, since we have taken $G_{3}=0$, a probe D7 is supersymmetric if it is holomorphically embedded into the geometry and the worldvolume field strength $f_{2}$ vanishes. In this case, we will take the D7 embedding to satisfy

$$
z^{3}=\mu
$$

where $\mu$ is a fixed positive real number. The perturbative treatment of the $\overline{\mathrm{D} 3} \mathrm{~s}$ will be valid for our probe analysis when $\mu \gg\left(\frac{p}{N}\right)^{1 / 8} L$. Note that this embedding preserves an SO (4) subgroup group of the $\mathrm{SO}(6)$ isometry of $R^{6}$. When $p \neq 0$, we expect that this embedding will no longer satisfy the D7 equations of motion. However, since the $\overline{\mathrm{D} 3} \mathrm{~s}$ do not break the isometries preserved by the D7, we expect the SO (4) symmetry to remain even after the branes are bent. It is convenient to rewrite the coordinates as ${ }^{9}$

$$
z^{1}=\mu u \cos \frac{\theta}{2} \mathrm{e}^{\mathrm{i}(2 \psi+\eta+\varphi) / 2}, \quad z^{2}=\mu u \sin \frac{\theta}{2} \mathrm{e}^{\mathrm{i}(2 \psi+\eta-\varphi) / 2}, \quad z^{3}=\mu(1+\chi) \mathrm{e}^{\mathrm{i} \psi},
$$

\footnotetext{
${ }^{9}$ There are more convenient coordinate definitions that we can make. However, this set will be more convenient when we include the backreaction of other D7-branes.
} 
in which $u \geq 0$ and $\chi \in[-1, \infty)$. This corresponds to

$$
\sin \frac{\gamma}{2}=\frac{\mu}{r}(1+\chi), \quad \cos \frac{\gamma}{2}=\frac{\mu}{r} u,
$$

and so

$$
r^{2}=\mu^{2}\left[(1+\chi)^{2}+u^{2}\right]
$$

Then the metric takes the form

$$
\mathrm{d} s_{6}^{2}=\mu^{2} \mathrm{e}^{2 B}\left\{\mathrm{~d} u^{2}+\mathrm{d} \chi^{2}+\left(u^{2}+(1+\chi)^{2}\right) \mathrm{d} \psi^{2}+\frac{u^{2}}{4}\left[\left(h^{1}\right)^{2}+\left(h^{2}\right)^{2}+\left(h^{3}\right)^{2}\right]+u^{2} \mathrm{~d} \psi h^{1}\right\},
$$

in which we have defined

$$
h^{1}=\mathrm{d} \eta+\cos \theta \mathrm{d} \varphi, \quad h^{2}=\sin \theta \mathrm{d} \varphi, \quad h^{3}=\mathrm{d} \theta .
$$

This effectively expresses $C^{3}$ as a foliation of surfaces of the type $z^{3}=\nu$ for $\nu \in C$. The embedding (3.9) corresponds to $\psi=0, \chi=0$ while more general embeddings respecting the isometries will have $\chi$ and $\psi$ as functions of $u$.

Our goal is to find the embedding that extremizes the probe brane action (A.6). In the presence of non-trivial $G_{3}$, such a configuration might be accompanied by non-vanishing worldvolume flux $f_{2}$. Since we will consider such a case later, we will include the $f_{2}$ terms in the D7 action, but from Lorentz invariance we can impose that the field strength has no legs in the external directions. Similarly, we can take the ansatz that the open-string fields depend only on $u$. With this ansatz, the CS contribution to the D7-brane action automatically vanishes and hence

$$
S_{\mathrm{D} 7}=-\tau_{\mathrm{D} 7} \int \mathrm{d}^{8} \xi \sqrt{\operatorname{det}\left(M_{a b}\right)},
$$

in which $M_{a b}$ is the internal generalized metric

$$
M_{a b}=\mathrm{P}[g]_{a b}+2 \pi \alpha^{\prime} \mathrm{e}^{2 A} f_{a b} .
$$

Writing the worldvolume gauge field as $a_{1}=a_{h^{i}} h^{i}$, we find

$$
\begin{aligned}
M_{u u} & =\mu^{2} \mathrm{e}^{2 B}\left\{1+\chi^{\prime 2}+\left(u^{2}+(1+\chi)^{2}\right) \psi^{\prime 2}\right\}, \\
M_{h^{i} h^{j}} & =\mu^{2} \mathrm{e}^{2 B} \frac{u^{2}}{4} \delta_{i j}, \\
M_{u h^{1}} & =\mu^{2} \mathrm{e}^{2 B} \frac{u^{2}}{2} \psi^{\prime}+2 \pi \alpha^{\prime} \mathrm{e}^{2 A} a_{h^{1}}^{\prime}, \\
M_{u h^{2}} & =2 \pi \alpha^{\prime} \mathrm{e}^{2 A} a_{h^{2}}^{\prime}, \\
M_{u h^{3}} & =2 \pi \alpha^{\prime} \mathrm{e}^{2 A} a_{h^{3}}^{\prime} .
\end{aligned}
$$

Then,

$$
S_{\mathrm{D} 7}=-\frac{\tau_{\mathrm{D} 7} \mu^{4}}{8} \int \mathrm{d}^{4} x \mathrm{~d} u \mathrm{~d}^{3} h \sqrt{W},
$$

in which $\mathrm{d}^{3} h:=h^{1} h^{2} h^{3}$ and

$$
W=u^{6} \mathrm{e}^{8 B}\left\{1+\chi^{\prime 2}+(1+\chi)^{2} \psi^{\prime 2}+\frac{4}{u^{2}} \frac{\left(2 \pi \alpha^{\prime}\right)^{2}}{\mu^{4}} \mathrm{e}^{4 A-4 B} a_{h^{i}}^{\prime} a_{h^{i}}^{\prime}\right\} .
$$


We first consider the equations of motion for $\psi$ and $a_{h^{i}}$. These are

$$
0=\partial_{u}\left[u^{6} \mathrm{e}^{8 B} \frac{(1+\chi)^{2}}{\sqrt{W}} \partial_{u} \psi\right], \quad 0=\partial_{u}\left[u^{4} \frac{\mathrm{e}^{4 A+4 B}}{\sqrt{W}} \partial_{u} a_{h^{i}}\right] .
$$

Hence we see that $\psi=0$ and $a_{h^{i}}=0$ are solutions whether or not $\overline{\mathrm{D} 3}$-branes are present. Inserting these solutions, the action becomes

$$
S_{\mathrm{D} 7}=-\frac{\tau_{\mathrm{D} 7} \mu^{4}}{8} \int \mathrm{d}^{4} x \mathrm{~d} u \mathrm{~d}^{3} h u^{3} \mathrm{e}^{4 B}\left(1+\chi^{\prime 2}\right)^{1 / 2} .
$$

The factor $\mathrm{e}^{4 B}$ is a function of $r$ and therefore of $\chi$ and $u$. Hence the embedding is determined by

$$
0=\partial_{u}\left[\frac{u^{3} \mathrm{e}^{4 B}}{\sqrt{1+\left(\partial_{u} \chi\right)^{2}}} \partial_{u} \chi\right]-u^{3}\left(\partial_{\chi} \mathrm{e}^{4 B}\right) \sqrt{1+\left(\partial_{u} \chi\right)^{2}} .
$$

Variation of the action also yields the boundary condition

$$
0=\left[\frac{u^{3} \mathrm{e}^{4 B}}{\sqrt{1+\left(\partial_{u} \chi\right)^{2}}} \partial_{u} \chi \delta \chi\right]_{u=0}^{u=\infty}
$$

We will satisfy this by requiring that the brane asymptotes to the unperturbed solution $\chi=0$ as $u \rightarrow \infty$, while at $u=0$ we impose

$$
0=\frac{u^{3} \mathrm{e}^{4 B}}{\sqrt{1+\left(\partial_{u} \chi\right)^{2}}} \partial_{u} \chi .
$$

When $B=0$, the solution to the equation of motion takes the form

$$
\chi=\tilde{c}_{1}+\int \mathrm{d} u \frac{\tilde{c}_{2}}{\sqrt{u^{6}-\tilde{c}_{2}^{2}}}
$$

and it is easy to see that the only solution satisfying the boundary conditions is $\tilde{c}_{1}=\tilde{c}_{2}=0$, reproducing the unperturbed supersymmetric embedding.

As anticipated, the equation of motion and boundary conditions do not depend directly on the warping, but only on the internal metric. The equation is nonlinear and difficult to solve. However, we can use the fact that the background is characterized by a small parameter $\frac{p}{N} \ll 1$ to solve the above equation perturbatively. To this end, we write

$$
\chi=\sum_{n=0}^{\infty}\left(\frac{p}{N}\right)^{n} \chi^{(n)}
$$

The zeroth order equation of motion is

$$
0=\frac{u^{3} \partial_{u}^{2} \chi^{(0)}}{\left[1+\left(\partial_{u} \chi^{(0)}\right)^{2}\right]^{3 / 2}}+\frac{3 u^{2} \partial_{u} \chi^{(0)}}{\left[1+\left(\partial_{u} \chi^{(0)}\right)^{2}\right]^{1 / 2}} .
$$

This is solved by (among other things) $\chi^{(0)}=0$, corresponding to the unperturbed embedding.

The next order equation is

$$
0=u^{3} \partial_{u}^{2} \chi^{(1)}+3 u^{2} \partial_{u} \chi^{(1)}-\frac{16 L^{8}}{5 \mu^{8}} \frac{u^{3}}{\left(1+u^{2}\right)^{5}},
$$




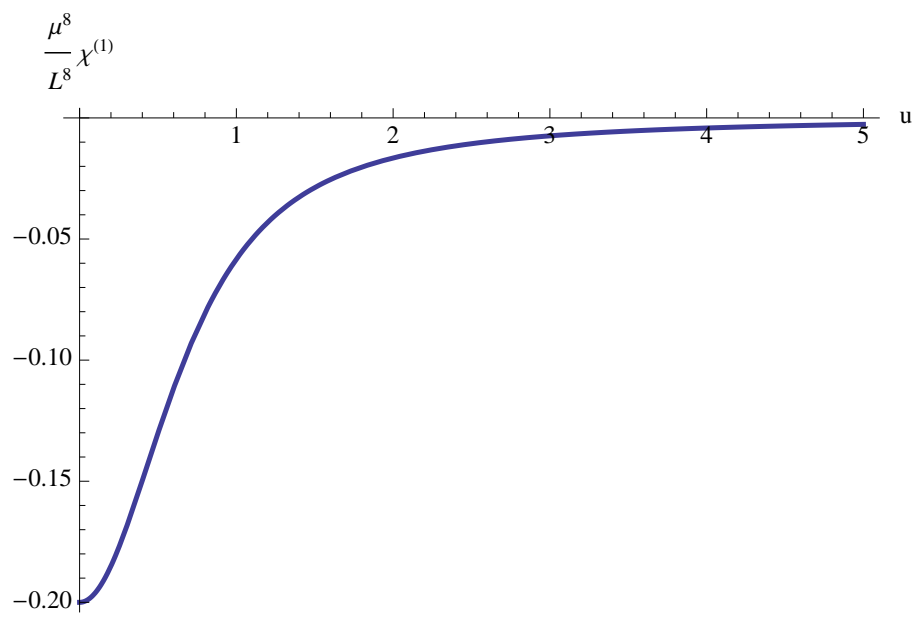

Figure 1. Profile of a D7 probe of $A d S^{5} \times S^{5}$ perturbed by $p \ll N$ D3- $\overline{\mathrm{D} 3}$ pairs. The curve is specified by $(3.30)$.

which has the solution

$$
\chi^{(1)}=c_{1}+\frac{2 L^{8}+c_{2} \mu^{8}\left(1+u^{2}\right)^{3}}{30 u^{2}\left(1+u^{2}\right)^{3} \mu^{8}} .
$$

Requiring $\chi \rightarrow 0$ as $u \rightarrow \infty$ sets $c_{1}=0$ while imposing (3.24) at $u=0$ sets $c_{2}=-2 L^{8} / \mu^{8}$, giving

$$
\chi^{(1)}=-\frac{L^{8}}{15 \mu^{8}} \frac{u^{4}+3 u^{2}+3}{\left(1+u^{2}\right)^{3}} .
$$

A plot of this function is shown in figure 1 .

The above solution naively indicates that at least part of the D7 falls toward the $\overline{\mathrm{D} 3} \mathrm{~s}$. However, we must be careful to disentangle the effect of the motion of the D7 branes and the fact that the backreaction of the $\overline{\mathrm{D} 3}$ changes the metric. That is, the physically relevant quantity is of course the change in proper distance. However, because the unperturbed geometry is anti-de Sitter, the proper distance between the D3-brane and the D7s branes is divergent. Equivalently, the dual theory is a conformal theory and so there is no natural scale against which to compare energies. We might consider measuring the unwarped distance (that is, using the internal metric $g_{m n}$ ) between the D7-brane and the D3-branes, but this quantity will be formally divergent in the perturbed geometry. Indeed the proper unwarped distance between two points on the same point on the $S^{5}$ is

$$
\Delta s=\int_{r_{1}}^{r_{2}} \mathrm{~d} r \mathrm{e}^{B}=r_{2}-r_{1}-\frac{\mathcal{S} L^{8}}{70} \frac{r_{2}^{7}-r_{1}^{7}}{r_{1}^{7} r_{2}^{7}}
$$

which diverges as either $r_{1}$ or $r_{2}$ approaches 0 . However, even if this were somehow convergent, the backreaction of the $\overline{\mathrm{D} 3}$ becomes large as $r \rightarrow 0$ and the solution will differ significantly from the perturbative one we work with here and so the result could not be trusted.

To avoid these issues, we consider two probe D7-branes, $\mathrm{D} 7_{1}$ and $\mathrm{D} 7_{2}$, which asymptote to the embeddings $z^{3}=\mu_{i=1,2}$. When $p=0$ the branes are parallel so the unwarped distance between them is simply $\mu_{2}-\mu_{1}$ (where we have taken $\mu_{2}>\mu_{1}$ for definiteness). To characterize the warped distance 


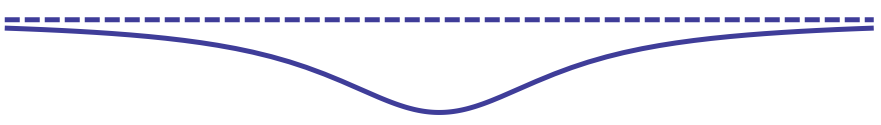

Figure 2. Sketch of two initially parallel branes (represented by the dashed lines) that are deformed by the presence of an attractive source. The lower brane experiences a greater attraction than the higher one, and hence the distance between the branes increases.

between them, we consider the points of the D7s that are closest to the D3s. These are simply where $r=\mu_{i}$ and the geodesic between the two points is a radial path. Then

$$
\Delta \hat{s}=\int_{\mu_{1}}^{\mu_{2}} \mathrm{e}^{-A+B} \mathrm{~d} r=L \log \frac{\mu_{2}}{\mu_{1}} .
$$

In the perturbed geometry, the minimal radii are, to leading order in $\frac{p}{N}$,

$$
r_{i}=\mu_{i}\left(1-\frac{p}{5 N} \frac{L^{8}}{\mu_{i}^{8}}\right)
$$

and the unwarped and warped distances between the branes are

$$
\begin{aligned}
& \Delta s=\mu_{2}-\mu_{1}+\frac{13 p L}{70 N}\left(\frac{L^{7}}{\mu_{1}^{7}}-\frac{L^{7}}{\mu_{2}^{7}}\right), \\
& \Delta \hat{s}=L \log \frac{\mu_{2}}{\mu_{1}}+\frac{17 p L}{80 N}\left(\frac{L^{8}}{\mu_{1}^{8}}-\frac{L^{8}}{\mu_{2}^{8}}\right) .
\end{aligned}
$$

For both of these measures, the proper distance between the branes increases when we take into account the perturbations. This is consistent with the fact that the branes are being attracted to non-BPS stack of branes; the D7 brane that begins closer to the $\overline{\mathrm{D} 3} \mathrm{~s}$ is attracted more strongly and so moves a greater distance than the D7 that is further away from them, leading to an increase in proper distance (see figure 2). We note however one subtlety in characterizing the movement using the warped distance: if the geometry is perturbed by just the addition of D3-branes, then the warped distance (3.32) increases even though the D3s do not exert a force on the D7s. The unwarped proper distance, which does not change, is therefore a more honest representation of the reaction of the D7s.

We can also characterize the influence of the $\overline{\mathrm{D} 3} \mathrm{~s}$ by the change in the energy density (that is, energy per unit volume of Minkowski space) of the D7. Since the configuration that we are considering is static, this is simply given by the Lagrangian density defined by $S=\int \operatorname{dvol}_{R^{3,1}} \mathcal{L}$,

$$
\Delta \mathcal{E}=-\Delta \mathcal{L} .
$$


Defining

$$
\mathcal{V}_{Y^{3}}=\int h^{1} h^{2} h^{3}
$$

and

$$
T=-\frac{\tau_{\mathrm{D} 7} \mu^{4}}{8} \mathcal{V}_{Y^{3}}
$$

the energy density is

$$
\mathcal{E}=T \int \mathrm{d} u u^{3} \mathrm{e}^{4 B} \sqrt{1+\chi^{\prime 2}}
$$

When $p=0, \chi=0$ and so the perturbed wavefunction only contributes to the energy at $\mathcal{O}\left(\frac{p^{2}}{N^{2}}\right)$. Since we are working in a non-compact geometry, $\mathcal{E}$ diverges. However, the change in the energy defined in this way is convergent

$$
\Delta \mathcal{E}=\mathcal{E}_{p \neq 0}-\mathcal{E}_{p=0}=-T\left\{\frac{p}{N} \frac{L^{8}}{30 \mu^{8}}+\frac{p^{2}}{N^{2}} \frac{L^{16}}{175 \mu^{16}}\right\} .
$$

If we artificially compactify the holographic coordinate by hand, this term would behave like a potential in the $4 \mathrm{~d}$ theory and indeed we see that it favors small values of $\mu$, indicating an attraction between the 3 -branes and the D7 that is absent in the $p=0$ case. Note that in computing the above quantity we have made use of the $\mathcal{O}\left(\frac{p^{2}}{N^{2}}\right)$ corrections to the geometry (3.2).

Finally, we comment briefly on the dual field theory analysis of the above behavior. As argued in [13], the open-string field $\chi$, which corresponds to transverse deformations of the brane, is dual to a field-theory operator with scaling dimension $\Delta=3$. We can confirm this fact quickly by observing that the linearized solutions of (3.27) scale as $u^{-2}$ and $u^{0}$ when $p=0$. At large $u$, we have $r \approx \mu u$ and hence the asymptotic solutions act as

$$
\chi \sim \chi_{\mathrm{dom}}+\chi_{\mathrm{sub}} r^{-2}
$$

Comparing this to the result for a canonically normalized scalar

$$
\Phi \sim \Phi_{\mathrm{dom}} r^{\Delta-4}+\Phi_{\mathrm{sub}} r^{-\Delta}
$$

and taking ratios of the terms (which corrects for the fact that $\chi$ is not canonically normalized) we recover $\Delta=3$. Evidently the canonically normalized field is proportional to $\chi / u$, a fact that is easily confirmed by expanding (3.21). A constant shift in $\chi$ is a solution to (3.27) (in the $p=0$ case), which is a reflection of the absence of a force between the D3s and the D7. Such a shift in $\mu$ results in a shift in the mass of the quarks of the dual theory. That is, the addition of $N_{f}$ D7-branes at $z^{3}=\mu$ corresponds to the addition of quarks, fields transforming as bifundamentals of $\mathrm{SU}(N) \times \mathrm{U}\left(N_{f}\right)$. The corresponding term in the superpotential is

$$
W \ni m_{Q} \tilde{Q} Q
$$

where $Q$ and $\tilde{Q}$ transform under conjugate representations, the mass is set by the embedding parameter, $m_{Q} \propto \mu$, and we have suppressed interactions between the quarks and the adjoint fields of the $\mathcal{N}=4$ sector. A constant shift of the D7s shifts $m_{Q}$ and hence $\chi$ is dual to the superpotential operator $\tilde{Q} Q$. In terms of the chiral superfields $\mathcal{Q} \sim Q+\theta \psi_{Q}+\theta^{2} F_{Q}, \chi$ corresponds to $\left.\tilde{\mathcal{Q}} \mathcal{Q}\right|_{\theta^{2}}$. For $p \neq 0$, the solution (3.30) scales as $u^{-2} \sim r^{-2}$ at large $u$, agreeing with the expected $r^{-3}$ scaling of the 
canonically normalized field, and hence the dual theory has a vacuum expectation value for $\left.\tilde{\mathcal{Q}} \mathcal{Q}\right|_{\theta^{2}}$ that is proportional to $\frac{p}{N}$.

The analysis of this section was performed for D7s with a finite embedding parameter (3.9). The case for vanishing $\mu$ cannot be immediately carried over since the coordinate system (3.10) made use of a finite $\mu$. Furthermore, the backreaction of the $\overline{\mathrm{D} 3}$-brane increases as we approach the brane and hence cannot treat the addition of the anti-branes as a perturbation to the geometry even when $\frac{p}{N} \ll 1$. However, following [48] we can argue that such a D7 brane will not be deformed in the sense that $z^{3}=0$ is still a solution to the D7-brane equations of motion in the presence of the $\overline{\mathrm{D} 3} \mathrm{~s}$. This argument is reviewed in a more general context in appendix C.1.

\subsection{Backreacting D7s}

In the previous section, we considered the backreaction of $N$ D3-branes coincident with $p \ll N$ D3-D3 pairs and argued that a D7 probe of such a geometry feels a small attractive force and hence bends toward the 3-branes. We should be able to observe such an interaction from the perspective of the 3-branes as well. This could in principle be accomplished by placing probe 3-branes in the geometry resulting from the backreaction of such a D7. Unfortunately, the backreaction of codimension-2 objects is difficult to compute except in particularly simple cases and the configuration we will ultimately consider is different from the one considered in the previous section so a direct comparison will not be possible.

One strategy to make progress on the problem is to consider the backreaction of a smeared distribution of D7-branes. That is, rather than considering the backreaction of a single D7 satisfying the embedding condition $z^{3}=\nu$, we consider $N_{f}$ D7-branes each of which satisfy an $\mathrm{SU}(3) \times \mathrm{U}(1)$ rotation of that embedding

$$
a_{i} z^{i}=\nu \mathrm{e}^{\mathrm{i} \alpha},
$$

in which $\alpha \in[0,2 \pi)$ and the $a_{i}$ are complex parameters satisfying $a_{i} \bar{a}_{\bar{i}}=1$. We then assume that $N_{f}$ is large and that the branes are placed in such a way that the distribution can, to good approximation, be treated as following from uniformly integrating over the $a_{i}$ and $\alpha$. The result for $p=0$ was found for $\nu=0$ in [44] and for $\nu \neq 0$ in [48]. For simplicity, we will focus on the $\nu=0$ case which we review in appendix C.1. The corresponding quarks in the dual theory are massless and so we will occasionally abuse terminology and refer to such D7s as massless D7s. With the appropriate smearing, the metric for the internal space can be written in the form

$$
\mathrm{d} s_{6}^{2}=\mathrm{e}^{2 B}\left\{\mathrm{~d} r^{2}+r^{2}(\mathrm{~d} \psi+\mathcal{A})^{2}\right\}+\mathrm{e}^{2 C} r^{2} \mathrm{~d} s_{C P^{2}}^{2} .
$$

The solution describing the backreaction of the smeared branes is

$$
\begin{aligned}
F_{1} & =-\frac{N_{f}}{2 \pi}(\mathrm{d} \psi+\mathcal{A}), \\
\mathrm{e}^{-\phi} & =\frac{1}{g_{\mathrm{s}}}\left(1-\frac{g_{\mathrm{s}} N_{f}}{2 \pi} \log \frac{r}{r_{\mathrm{s}}}\right), \\
\mathrm{e}^{2 B} & =\left(1-\frac{g_{\mathrm{s}} N_{f}}{2 \pi}\left[\log \frac{r}{r_{\mathrm{s}}}-\frac{1}{6}\right]\right)^{1 / 3}, \\
\mathrm{e}^{2 C} & =g_{\mathrm{s}} \mathrm{e}^{-\phi-4 B}, \\
\Phi_{+}^{-1} & =-8 \pi g_{\mathrm{s}} N \alpha^{\prime 2} \int_{r_{\mathrm{LP}}}^{r} \frac{\mathrm{d} x}{x^{5}} \mathrm{e}^{-4 B(x)} .
\end{aligned}
$$


Here, $r_{\mathrm{s}}$ is an arbitrarily chosen scale such that $\phi\left(r_{\mathrm{s}}\right)=\log g_{\mathrm{s}}$. Note that the dual theory exhibits a Landau pole at $r_{\mathrm{LP}}=r_{\mathrm{s}} \mathrm{e}^{2 \pi / g_{\mathrm{s}} N_{f}}$ where $\mathrm{e}^{-\phi}=0$.

We now consider an $\overline{\mathrm{D} 3}$ probe of the above geometry. In the absence of D7-branes, the $\overline{\mathrm{D} 3}$ will feel a force from the D3 charge given by ${ }^{10}$

$$
F=-\tau_{\mathrm{D} 3} \frac{\mathrm{d} \Phi_{+}}{\mathrm{d} r}=-8 \tau_{\mathrm{D} 3} \frac{r^{3}}{L^{4}} .
$$

In order to make a precise comparison of the potential before and after the addition of D7-branes, we must account for the fact that both $\Phi_{+}$and the metric factor $g_{r r}$ are modified when $N_{f}=0$ is finite. Said differently, from the point of view of the worldvolume field theory of the $\overline{\mathrm{D} 3}$, the radial position is a scalar field that has different normalizations before and after the introduction of D7s and so to make a comparison of the potentials we ought to give the fields the same normalization. To leading order in momenta along the $\overline{\mathrm{D} 3}$ worldvolume, the action for the $\overline{\mathrm{D} 3}$ is

$$
S_{\overline{\mathrm{D} 3}}=-\tau_{\mathrm{D} 3} \int \mathrm{d}^{4} x\left\{\frac{1}{2} \mathrm{e}^{4 A+2 B} \eta^{\mu \nu} \partial_{\mu} r \partial_{\nu} r+\Phi_{+}(r)\right\},
$$

where we have now considered an $\overline{\mathrm{D} 3}$-brane that is moving entirely in the radial direction so that the position of the brane is described by $r\left(x^{\mu}\right)$. When $N_{f}=0$, this gives

$$
S_{\overline{\mathrm{D} 3}}=-\tau_{\mathrm{D} 3} \int \mathrm{d}^{4} x\left\{\frac{1}{2} \frac{r^{4}}{L^{4}} \eta^{\mu \nu} \partial_{\mu} r \partial_{\nu} r+\frac{2 r^{4}}{L^{4}}\right\} .
$$

From the perspective of the $\overline{\mathrm{D} 3}$-brane, the canonically normalized field is

$$
\sigma=\sqrt{\tau_{\mathrm{D} 3}} \frac{r^{3}}{3 L^{2}}
$$

in terms of which the potential is

$$
V(\sigma)=2 \cdot 3^{4 / 3} \frac{\tau_{\mathrm{D} 3}^{1 / 3}}{L^{4 / 3}} \sigma^{4 / 3} .
$$

When $N_{f}>0$, we cannot analytically find such a canonically normalized field. However when $\frac{g_{\mathrm{s}} N_{f}}{2 \pi}\left|\log \frac{r}{r_{\mathrm{s}}}\right| \ll 1$ the backreaction of the D7 can be treated perturbatively and we have

$$
\mathrm{e}^{2 B} \approx\left(1-\frac{g_{\mathrm{s}} N_{f}}{6 \pi}\left[\log \frac{r}{r_{\mathrm{s}}}+\frac{1}{3}\right]\right)
$$

where we have taken $\frac{g_{s} N_{f}}{2 \pi}$ to be small and have neglected terms of higher order in that parameter. Then,

$$
\Phi_{+} \approx \frac{2 r^{4}}{L^{4}}\left(1-\frac{g_{\mathrm{s}} N_{f}}{3 \pi}\left[\log \frac{r}{r_{\mathrm{s}}}+\frac{1}{12}\right]\right) .
$$

Hence to this approximation

$$
S_{\overline{\mathrm{D} 3}}=-\tau_{\mathrm{D} 3} \int \mathrm{d}^{4} x\left\{\frac{1}{2} \frac{r^{4}}{L^{4}}\left(1-\frac{g_{\mathrm{s}} N_{f}}{2 \pi} \log \frac{r}{r_{\mathrm{s}}}\right) \eta^{\mu \nu} \partial_{\mu} r \partial_{\nu} r+\frac{2 r^{4}}{L^{4}}\left(1-\frac{g_{\mathrm{s}} N_{f}}{3 \pi}\left[\log \frac{r}{r_{\mathrm{s}}}+\frac{1}{12}\right]\right)\right\} .
$$

\footnotetext{
${ }^{10}$ Note that this expression is slightly different, but not inconsistent with, that appearing in [42] since the authors there consider the interaction between a stationary $\overline{\mathrm{D} 3}$ and a mobile, distant, D3 brane. In contrast, here we are considering a mobile $\overline{\mathrm{D} 3}$ interacting only with the background charge of $A d S^{5} \times S^{5}$.
} 
We can construct a normalized field $\sigma$ in terms of which

$$
r=\left(\frac{3 L^{2}}{\sqrt{\tau_{\mathrm{D} 3}}} \sigma\right)^{1 / 3}\left(1+\frac{g_{\mathrm{s}} N_{f}}{36 \pi}\left[\log \frac{3 L^{2} \sigma}{\sqrt{\tau_{\mathrm{D} 3}} r_{\mathrm{s}}^{3}}-\frac{1}{2}\right]\right)
$$

and the potential is

$$
V(\sigma)=2 \cdot 3^{4 / 3} \frac{\tau_{\mathrm{D} 3}^{1 / 3}}{L^{4 / 3}}\left(1-\frac{g_{\mathrm{s}} N_{f}}{12 \pi}\right) \sigma^{4 / 3} .
$$

Hence the potential experienced by an $\overline{\mathrm{D} 3}$ is reduced by the presence of the D7s. Note that, at least to this order of approximation, the result is independent of the integration constant $r_{\mathrm{s}}$. A useful check of our results would be to confirm that (3.55) is consistent with the results of the probe D7 analysis section 3.1. However, in the probe D7 analysis, the flavor branes were placed at larger radii than the $\overline{\mathrm{D} 3}$-branes while in the analysis leading to (3.55), the $\overline{\mathrm{D} 3}$-branes were placed at larger $r$. Hence (3.55) cannot be used to confirm the probe D7 analysis. Due to the smearing procedure that we used, the force on an $\overline{\mathrm{D} 3}$-brane placed at smaller $r$ would not be modified by the presence of the flavor branes, and so to check the probe D7 analysis we would need to move beyond the smeared approximation, an analysis which is beyond the scope of our current work.

Finally, we can consider a D3 probe of the geometry resulting from the backreaction of $\overline{\mathrm{D} 3} \mathrm{~s}$ and D7s. The precise thing to do would be to backreact the perturbed D7 branes which requires solving the D7 equations of motion together with the supergravity equations of motion. However analogous to what was argued in [48], D7-branes characterized by $\nu=0$ are not perturbed by the addition of $\overline{\mathrm{D} 3}$ branes even once their smeared backreaction is taken into account as well. Even still, the backreaction of such branes is difficult to compute. We can make progress by considering the (admittedly nongeneric) case in which the $\overline{\mathrm{D} 3} \mathrm{~s}$ and the D7s can be treated as comparable perturbations. Writing $\delta_{p}=\frac{p}{N}$ and $\delta_{f}=\frac{g_{\mathrm{s}} N_{f}}{2 \pi}$, the corrections resulting from both $\overline{\mathrm{D} 3 \mathrm{~s}}$ and D7s comes in at $\mathcal{O}\left(\delta_{p} \delta_{f}\right)$ and hence we must use the solution through $\mathcal{O}\left(\delta^{2}\right)$ where we assume $\delta \sim \delta_{p} \sim \delta_{f}$. The resulting solution should then be valid when $\min \left(\delta^{1 / 8} L, r_{\mathrm{s}} \mathrm{e}^{-1 / \delta}\right) \ll r \ll r_{\mathrm{s}} \mathrm{e}^{1 / \delta}$. Consistency of this condition requires us to chose $r_{\mathrm{s}}$ such that $r_{\mathrm{s}} \gg \delta^{1 / 8} L \mathrm{e}^{-1 / \delta}$ and hence $L \delta^{1 / 8} \gg r_{\mathrm{s}} \mathrm{e}^{-1 / \delta}$. Thus the solution is valid when $L \delta^{1 / 8} \ll r \ll r_{\mathrm{s}} \mathrm{e}^{1 / \delta}$. The result is (see appendix C.1)

$$
\begin{aligned}
\mathrm{e}^{4 A}= & \frac{L^{4}}{r^{4}}+\delta_{f} \frac{2}{3} \frac{L^{4}}{r^{4}}\left(\log \frac{r}{r_{\mathrm{s}}}+\frac{1}{12}\right)+\delta_{p} \frac{4}{5} \frac{L^{12}}{r^{12}}+\delta_{f}^{2} \frac{5}{9} \frac{L^{4}}{r^{4}}\left(\log ^{2} \frac{r}{r_{\mathrm{s}}}+\frac{1}{6} \log \frac{r}{r_{\mathrm{s}}}+\frac{5}{72}\right) \\
& +\delta_{f} \delta_{p} \frac{8}{5} \frac{L^{12}}{r^{12}}\left(\log \frac{r}{r_{\mathrm{s}}}+\frac{1}{12}\right)+\delta_{p}^{2} \frac{54}{125} \frac{L^{20}}{r^{20}}, \\
\omega= & \frac{r^{4}}{L^{4}}-\delta_{f} \frac{2}{3} \frac{r^{4}}{L^{4}}\left(\log \frac{r}{r_{\mathrm{s}}}+\frac{1}{12}\right)+\delta_{p} \frac{6}{5} \frac{L^{4}}{r^{4}}-\frac{1}{9} \delta_{f}^{2} \frac{r^{4}}{L^{4}}\left(\log ^{2} \frac{r}{r_{\mathrm{s}}}+\frac{1}{6} \log \frac{r}{r_{\mathrm{s}}}+\frac{23}{72}\right) \\
& +\frac{4}{5} \delta_{f} \delta_{p} \frac{L^{4}}{r^{4}}\left(\log \frac{r}{r_{\mathrm{s}}}+\frac{1}{12}\right)-\delta_{p}^{2} \frac{24}{125} \frac{L^{12}}{r^{12}}, \\
F_{1}= & -\frac{1}{g_{\mathrm{s}}} \delta_{f}(\mathrm{~d} \psi+\mathcal{A}), \\
\mathrm{e}^{-\phi}= & \frac{1}{g_{\mathrm{s}}}\left(1-\delta_{f} \log \frac{r}{r_{\mathrm{s}}}+\delta_{f} \delta_{p} \frac{1}{10} \frac{L^{8}}{r^{8}}\right), \\
\mathrm{e}^{2 B}= & -\delta_{f} \frac{1}{3}\left(\log \frac{r}{r_{\mathrm{s}}}+\frac{1}{3}\right)-\delta_{p} \frac{1}{5} \frac{L^{8}}{r^{8}}-\delta_{f}^{2} \frac{1}{9}\left(\log ^{2} \frac{r}{r_{\mathrm{s}}}+\frac{2}{3} \log \frac{r}{r_{\mathrm{s}}}-\frac{5}{36}\right) \\
& -\delta_{f} \delta_{p} \frac{1}{5} \frac{L^{8}}{r^{8}} \log \frac{r}{r_{\mathrm{s}}}-\delta_{p}^{2} \frac{1}{50} \frac{L^{16}}{r^{16}},
\end{aligned}
$$




$$
\begin{aligned}
\mathrm{e}^{2 C}= & -\delta_{f} \frac{1}{3}\left(\log \frac{r}{r_{\mathrm{s}}}-\frac{1}{6}\right)-\delta_{p} \frac{1}{5} \frac{L^{8}}{r^{8}}-\delta_{f}^{2} \frac{1}{9}\left(\log ^{2} \frac{r}{r_{\mathrm{s}}}-\frac{1}{3} \log \frac{r}{r_{\mathrm{s}}}+\frac{1}{36}\right) \\
& -\delta_{f} \delta_{p} \frac{1}{5} \frac{L^{8}}{r^{8}}\left(\log \frac{r}{r_{\mathrm{s}}}+\frac{1}{6}\right)-\delta_{p}^{2} \frac{1}{50} \frac{L^{16}}{r^{16}} .
\end{aligned}
$$

Here, we have suppressed the additive constants to $\mathrm{e}^{4 A}$ and $\omega$ which are present to ensure that $\mathrm{e}^{-4 A}$ and $\frac{1}{\omega}$ vanish at the Landau pole.

The relevant action for calculating the D3 potential is (3.47) with the replacement $\Phi_{+} \rightarrow \Phi_{-}$. Then, in terms of a canonically normalized field $\sigma$, the potential is

$$
V(\sigma)=-\delta_{p} \frac{2}{3^{4 / 3}} \frac{\tau_{\mathrm{D} 3}^{5 / 3} L^{4 / 3}}{\sigma^{4 / 3}}-\delta_{p} \delta_{f} \frac{1}{3^{7 / 3}} \frac{\tau_{\mathrm{D} 3}^{5 / 3} L^{4 / 3}}{\sigma^{4 / 3}}-\delta_{p}^{2} \frac{2}{405} \frac{L^{4} \tau_{\mathrm{D} 3}^{3}}{\sigma^{4}} .
$$

Hence the presence of the D7s results in a steeper potential for a probe D3.

We again note that the above configurations of D7s and 3-branes are not quite what we would want to consider to compliment the discussion in section 3.1. In the earlier analysis, the 3-branes were located at smaller radii than the D7s while in this section the bottom of the D7s is localized at smaller $r$ than the 3-branes. Unfortunately, the reverse situation is more difficult to describe. The backreaction of smeared D7-branes characterized by $\nu>0$ is non-trivial only for $r>\nu$ and hence the influence of such massive branes on 3-branes at $r=0$ cannot be captured in the smeared approximation.

\subsection{Forces between flavors}

In the absence of $\overline{\mathrm{D} 3}$-branes, parallel D7s in the above background will not feel a force between them. However, this will not be the case once supersymmetry is broken by the presence of the anti-branes. To be more precise, we consider two D7s that asymptote to $z^{3}=\mu_{1,2}$ and take $\left|\mu_{2}\right|>\left|\mu_{1}\right|$. When $p=0$, the embeddings $z^{3}=\mu$ will solve the equations of motion precisely, even when the backreaction of the D7s are taken into account due to the supersymmetry of the configuration. However, when the $\overline{\mathrm{D} 3} \mathrm{~s}$ backreact, this cancellation no longer occurs.

We can be more quantitative by considering a D7 probe of the geometry (3.56) describing the backreaction of smeared massless D7s and $\overline{\mathrm{D} 3} \mathrm{~s}$ in $A d S^{5} \times S^{5}$. In order for the perturbative treatment of the D7 backreaction to be valid, we must again consider the situation in which $\left|\log \frac{\mu}{r_{\mathrm{s}}}\right| \ll \frac{1}{\delta_{f}}$. That is, the embedding parameter of our probe D7 is neither too close to the origin nor too close to the Landau pole. Of course, since the D7 extends to the UV in the radial direction, there will be part of the probe worldvolume that reaches the Landau pole; however, at that point the influence of the $\overline{\mathrm{D} 3} \mathrm{~s}$ is small so that the configuration is approximately supersymmetric and the deviation from the $z^{3}=\mu$ embedding is expected to be negligible, a fact that we will confirm.

The process that we follow is largely the same as without the backreacting D7s. We again adopt the coordinates (3.10) in terms of which the metric (3.44) takes the form

$$
\mathrm{d} s_{6}^{2}=\frac{\mathrm{e}^{2 B}-\mathrm{e}^{2 C}}{r^{2}} \bar{z}^{\bar{A}} \mathrm{~d} z^{A} z^{B} \mathrm{~d} \bar{z}^{\bar{B}}+\mathrm{e}^{2 C} \mathrm{~d} z^{A} \mathrm{~d} \bar{z}^{\bar{A}}
$$


or

$$
\begin{aligned}
\mathrm{d} s_{6}^{2}=\mu^{2}\{ & \frac{u^{2} \mathrm{e}^{2 B}+(1+\chi)^{2} \mathrm{e}^{2 C}}{u^{2}+(1+\chi)^{2}} \mathrm{~d} u^{2}+\frac{u^{2} \mathrm{e}^{2 C}+(1+\chi)^{2} \mathrm{e}^{2 B}}{u^{2}+(1+\chi)^{2}} \mathrm{~d} \chi^{2}+\mathrm{e}^{2 B}\left[u^{2}+(1+\chi)^{2}\right] \mathrm{d} \psi^{2} \\
& +\frac{u^{2}}{4}\left(\frac{u^{2} \mathrm{e}^{2 B}+(1+\chi)^{2} \mathrm{e}^{2 C}}{u^{2}+(1+\chi)^{2}}\left(h^{1}\right)^{2}+\mathrm{e}^{2 C}\left(h^{2}\right)^{2}+\mathrm{e}^{2 C}\left(h^{3}\right)^{2}\right)+\mathrm{e}^{2 B} u^{2} \mathrm{~d} \psi h^{1} \\
& \left.+\frac{2 u(1+\chi)\left(\mathrm{e}^{2 B}-\mathrm{e}^{2 C}\right)}{u^{2}+(1+\chi)^{2}} \mathrm{~d} u \mathrm{~d} \chi\right\} .
\end{aligned}
$$

Then the internal generalized metric on the D7 probe is

$$
\begin{aligned}
& M_{u u}= \mu^{2}\left\{\frac{u^{2} \mathrm{e}^{2 B}+(1+\chi)^{2} \mathrm{e}^{2 C}}{u^{2}+(1+\chi)^{2}}+\frac{2 u(1+\chi)\left(\mathrm{e}^{2 B}-\mathrm{e}^{2 C}\right)}{u^{2}+(1+\chi)^{2}} \chi^{\prime}\right. \\
&\left.\quad+\frac{u^{2} \mathrm{e}^{2 C}+(1+\chi)^{2} \mathrm{e}^{2 B}}{u^{2}+(1+\chi)^{2}} \chi^{\prime 2}+\mathrm{e}^{2 B}\left(u^{2}+(1+\chi)^{2}\right) \psi^{\prime 2}\right\}, \\
& M_{h^{1} h^{1}}= \mu^{2} \frac{u^{2} \mathrm{e}^{2 B}+(1+\chi)^{2} \mathrm{e}^{2 C}}{u^{2}+(1+\chi)^{2}} \frac{u^{2}}{4}, \\
& M_{h^{2} h^{2}=}=M_{h^{3} h^{3}}=\mu^{2} \mathrm{e}^{2 C} \frac{u^{4}}{4}, \\
& M_{u h^{1}}=\mu^{2} \mathrm{e}^{2 B} \frac{u^{2}}{2} \psi^{\prime}+2 \pi \alpha^{\prime} g_{\mathrm{s}}^{1 / 2} \mathrm{e}^{2 A-\phi / 2} a_{h^{1}}^{\prime}, \\
& M_{u h^{2}}=2 \pi \alpha^{\prime} g_{\mathrm{s}}^{1 / 2} \mathrm{e}^{2 A-\phi / 2} a_{h^{2}}^{\prime}, \\
& M_{u h^{3}}=2 \pi \alpha^{\prime} g_{\mathrm{s}}^{1 / 2} \mathrm{e}^{2 A-\phi / 2} a_{h^{3}}^{\prime} .
\end{aligned}
$$

This gives the DBI action

$$
S_{\mathrm{D} 7}^{\mathrm{DBI}}=-\frac{\tau_{\mathrm{D} 7} \mu^{4}}{8} \int \mathrm{d}^{4} x \mathrm{~d} u \mathrm{~d}^{3} h \sqrt{W},
$$

where now

$$
W=W_{0}+W_{1} \chi^{\prime}+W_{2} \chi^{\prime 2}+Y \psi^{\prime 2}+H_{i j} a_{h^{i}}^{\prime} a_{h^{j}}^{\prime},
$$

with

$$
\begin{aligned}
W_{0} & =g_{\mathrm{s}}^{-2} \mathrm{e}^{4 C+2 \phi} \frac{u^{6}\left(u^{2} \mathrm{e}^{2 B}+(1+\chi)^{2} \mathrm{e}^{2 C}\right)^{2}}{\left(u^{2}+(1+\chi)^{2}\right)^{2}}, \\
W_{1} & =g_{\mathrm{s}}^{-2} \mathrm{e}^{4 C+2 \phi} \frac{2 u^{7}(1+\chi)\left(\mathrm{e}^{2 B}-\mathrm{e}^{2 C}\right)\left(u^{2} \mathrm{e}^{2 B}+(1+\chi)^{2} \mathrm{e}^{2 C}\right)}{\left(u^{2}+(1+\chi)^{2}\right)}, \\
W_{2} & =g_{\mathrm{s}}^{-2} \mathrm{e}^{4 C+2 \phi} \frac{u^{6}\left(u^{2} \mathrm{e}^{2 C}+(1+\chi)^{2} \mathrm{e}^{2 B}\right)\left(u^{2} \mathrm{e}^{2 B}+(1+\chi)^{2} \mathrm{e}^{2 C}\right)}{\left(u^{2}+(1+\chi)^{2}\right)^{2}}, \\
Y & =g_{\mathrm{s}}^{-2} \mathrm{e}^{2 B+6 C+2 \phi} u^{6}(1+\chi)^{2}, \\
H_{11} & =\frac{\left(2 \pi \alpha^{\prime}\right)^{2}}{\mu^{4}} 4 u^{4} g_{\mathrm{s}}^{-1} \mathrm{e}^{4 A+4 C+\phi}, \\
H_{22}=H_{33} & =\frac{\left(2 \pi \alpha^{\prime}\right)^{2}}{\mu^{4}} 4 u^{4} g_{\mathrm{s}}^{-1} \mathrm{e}^{4 A+4 C+\phi} \frac{u^{2} \mathrm{e}^{2 B-2 C}+(1+\chi)^{2}}{u^{2}+(1+\chi)^{2}}
\end{aligned}
$$


and other components of $H_{i j}$ vanishing.

In addition to the DBI action, there is a contribution from the Chern-Simons action due to the nontrivial $C_{0}$ sourced by the backreacting D7s. D7s couple magnetically to $C_{0}$ and so we construct

$$
F_{9}=-g_{\mathrm{s}}^{-2} \mathrm{e}^{2 \phi} \hat{*} \mathrm{~d} F_{1}=-Q_{f} \frac{r^{3}}{2} g_{\mathrm{s}}^{-2} \mathrm{e}^{2 \phi} \mathrm{e}^{4 C} \operatorname{dvol}_{R^{3,1}} \wedge \mathrm{d} r \wedge \mathcal{J} \wedge \mathcal{J}
$$

where we have written $F_{1}=-Q_{f}(\mathrm{~d} \psi+\mathcal{A})$ and made use of the fact that the flat space volume element is

$$
\operatorname{dvol}_{C^{3}}=\frac{1}{3 !} J \wedge J \wedge J=\frac{1}{2} r^{5} \mathrm{~d} r \wedge(\mathrm{d} \psi+\mathcal{A}) \wedge \mathcal{J} \wedge \mathcal{J},
$$

where the factor of $\frac{1}{2}$ comes from the fact that $\mathrm{dvol}_{C P^{2}}=\frac{1}{2} \mathcal{J} \wedge \mathcal{J}$. In the absence of 3-form flux, we have $F_{9}=\mathrm{d} C_{8}$ and so we can take

$$
C_{8}=Q_{f} \frac{r^{3}}{4} g_{\mathrm{s}}^{-2} \mathrm{e}^{2 \phi+4 C} \mathrm{dvol}_{R^{3,1}} \wedge \mathrm{d} r \wedge \mathcal{A} \wedge \mathcal{J}
$$

In terms of the coordinates (3.10), this is

$$
C_{8}=-Q_{f} \frac{\mu^{4} u^{4}}{32\left(u^{2}+(1+\chi)^{2}\right)} g_{\mathrm{s}}^{-2} \mathrm{e}^{2 \phi+4 C} \mathrm{dvol}_{R^{3,1}} \wedge[u \mathrm{~d} u+(1+\chi) \mathrm{d} \chi] \wedge \mathrm{d} \eta \wedge \sin \theta \mathrm{d} \theta \wedge \mathrm{d} \varphi,
$$

from which we easily calculate the pullback to the probe D7.

$$
\mathrm{P}\left[C_{8}\right]=-Q_{f} \frac{\mu^{4} u^{4}\left(u+(1+\chi) \chi^{\prime}\right)}{32\left(u^{2}+(1+\chi)^{2}\right)} g_{\mathrm{s}}^{-2} \mathrm{e}^{2 \phi+4 C} \operatorname{dvol}_{R^{3,1}} \wedge \mathrm{d} u \wedge \mathrm{d} \eta \wedge \mathrm{d} \theta \wedge \mathrm{d} \varphi .
$$

When $p=0$ the D7-brane is supersymmetric implying that the volume of the 4-cycle transverse to $R^{3,1}$ is calibrated in the sense that

$$
\operatorname{dvol}_{\Sigma^{4}}=-\frac{1}{2} \mathrm{P}[J \wedge J] \sim+\mathrm{d} u \wedge \mathrm{d} \eta \wedge \mathrm{d} \theta \wedge \mathrm{d} \varphi
$$

Although the volume form will not be the same for $p \neq 0$, this defines the orientation for a D7 (an $\overline{\mathrm{D} 7}$ has the opposite orientation). With this orientation, the CS contribution to the D7 action is

$$
S_{\mathrm{D} 7}^{\mathrm{CS}}=-\frac{\mu^{4} \tau_{\mathrm{D} 7} g_{\mathrm{s}}}{8} \int \mathrm{d}^{4} x \mathrm{~d} u \mathrm{~d}^{3} h \frac{u^{4}\left(u+(1+\chi) \chi^{\prime}\right)}{4\left(u^{2}+(1+\chi)^{2}\right)} Q_{f} g_{\mathrm{s}}^{-2} \mathrm{e}^{2 \phi+4 C} .
$$

The total action is then

$$
S_{\mathrm{D} 7}=-\frac{\mu^{4} \tau_{\mathrm{D} 7}}{8} \int \mathrm{d}^{4} x \mathrm{~d} u \mathrm{~d}^{3} h\left\{\sqrt{W}+\frac{u^{4}\left(u+(1+\chi) \chi^{\prime}\right)}{4\left(u^{2}+(1+\chi)^{2}\right)} Q_{f} g_{\mathrm{s}}^{-1} \mathrm{e}^{2 \phi+4 C}\right\} .
$$

It is easy to see that $\psi=a_{h^{i}}=0$ is again a solution to the equations of motion. Setting these fields on shell, the action takes the form

$$
S_{\mathrm{D} 7}=-\frac{\mu^{4} \tau_{\mathrm{D} 7}}{8} \int \mathrm{d}^{4} x \mathrm{~d} u \mathrm{~d}^{3} h\left\{\sqrt{W_{0}+W_{1} \chi^{\prime}+W_{2} \chi^{\prime 2}}+W_{3}+W_{4} \chi^{\prime}\right\},
$$


Then the equation of motion determining the perturbed embedding is

$$
\begin{aligned}
0= & \partial_{u}\left[\frac{W_{2}}{\sqrt{W}} \partial_{u} \chi\right]-\frac{1}{2} \frac{\partial_{\chi} W_{2}}{\sqrt{W}} \chi^{\prime 2}-\frac{1}{2} \frac{\partial_{\chi} W_{1}}{\sqrt{W}} \chi^{\prime}-\partial_{\chi} W_{4} \chi^{\prime} \\
& +\partial_{u}\left[\frac{W_{1}}{2 \sqrt{W}}\right]+\partial_{u} W_{4}-\frac{\partial_{\chi} W_{0}}{2 \sqrt{W}}-\partial_{\chi} W_{3} .
\end{aligned}
$$

The variation of the action also leads to the boundary terms ${ }^{11}$

$$
0=\left[\left(\frac{W_{2}}{\sqrt{W}} \chi^{\prime}+\frac{W_{1}}{2 \sqrt{W}}+W_{4}\right) \delta \chi\right]_{u=0}^{u=\infty}
$$

We again satisfy this by imposing that $\chi$ vanishes as $u \rightarrow \infty$ and imposing the Neumann condition at $u=0$.

Performing an expansion in $\delta \sim \delta_{f} \sim \delta_{p}$ in both $W_{i}$ and $\chi$ schematically as

$$
\chi=\sum_{n=0}^{\infty} \delta^{n} \chi^{(n)}
$$

the $\mathcal{O}\left(\delta^{0}\right)$ and $\mathcal{O}\left(\delta^{1}\right)$ corrections are the same as they were in the $N_{f}=0$ case of section 3.1. This is a manifestation of the fact that the D7 probe and the backreacting D7s are mutually supersymmetric when $p=0$. Hence through $\mathcal{O}\left(\delta^{1}\right)$ we again have (3.30)

$$
\chi=-\delta_{p} \frac{L^{8}}{15 \mu^{8}} \frac{u^{4}+3 u^{2}+3}{\left(1+u^{2}\right)^{3}} .
$$

The $\mathcal{O}\left(\delta^{2}\right)$ corrections to the equations of motion then take the form

$$
\begin{aligned}
0= & \delta^{2}\left[u^{3}\left(\chi^{(2)}\right)^{\prime \prime}+3 u^{2}\left(\chi^{(2)}\right)^{\prime}\right]+\delta_{p}^{2} \frac{16 L^{16}}{75 \mu^{16}} \frac{u^{3}}{\left(1+u^{2}\right)^{9}}\left(3 u^{6}+2 u^{4}-12 u^{2}-33\right) \\
& -\delta_{p} \delta_{f} \frac{64 L^{8}}{15 \mu^{8}} \frac{u^{3}}{\left(1+u^{2}\right)^{5}}\left[\log \frac{\sqrt{1+u^{2}} \mu}{r_{\mathrm{s}}}-\frac{u^{8}+6 u^{6}+14 u^{4}-2 u^{2}-26}{96\left(1+u^{2}\right)}\right] .
\end{aligned}
$$

With the above boundary conditions the solution is

$$
\begin{aligned}
\chi= & -\delta_{p} \frac{L^{8}}{15 \mu^{8}} \frac{u^{4}+3 u^{2}+3}{\left(1+u^{2}\right)^{3}} \\
& -\delta_{p}^{2} \frac{L^{16}}{7875 \mu^{2}} \frac{270 u^{12}+1890 u^{10}+5670 u^{8}+9450 u^{6}+9513 u^{4}+5761 u^{2}+1813}{\left(1+u^{2}\right)^{7}} \\
& -\delta_{p} \delta_{f} \frac{L^{8}}{1080 \mu^{8}} \frac{1}{\left(1+u^{2}\right)^{4}}\left\{37 u^{6}+160 u^{4}+262 u^{2}+148+96\left(u^{6}+4 u^{4}+6 u^{2}+3\right) \log \frac{\mu}{r_{\mathrm{s}}}\right. \\
& \left.-12\left(u^{6}+4 u^{4}+6 u^{2}+8+\frac{5}{u^{2}}\right) \log \left(1+u^{2}\right)\right\} .
\end{aligned}
$$

\footnotetext{
${ }^{11}$ Note that the $u=\infty$ boundary condition is imposed where there is perturbative control which fails at around the Landau pole at $r_{\mathrm{LP}}=r_{\mathrm{s}} \mathrm{e}^{2 \pi / g_{\mathrm{s}} N_{f}}$. Due to the corresponding UV singularity, a more appropriate boundary condition to impose might be that $\chi$ vanishes at some finite $r \lesssim r_{\mathrm{LP}}$ as in [49]. However, since for $\delta_{f} \ll 1$ this occurs at exponentially large radii, the difference between formally imposing a boundary condition at $u=\infty$ and this finite part will be exponentially small. A similar problem does not occur for the $u=0$ boundary condition since $u=0$ corresponds to $r \approx \mu$ which we are choosing to be within the region of control.
} 
From this we see that the minimal coordinate value reached by the $\mathrm{D} 7$ is

$$
r_{\min }=\mu\left\{1-\delta_{p} \frac{L^{8}}{5 \mu^{8}}\left[1+\delta_{p} \frac{259 L^{8}}{225 \mu^{8}}+\delta_{f} \frac{4}{3}\left(\log \frac{\mu}{r_{\mathrm{s}}}+\frac{11}{36}\right)\right]\right\} .
$$

Hence whether or not the backreacting D7s attract or repel the probe depends on the ratio $\frac{\mu}{r_{\mathrm{s}}}$. The backreacting D7s attract D7 probes so long as $\mu \geq r_{\mathrm{s}} \mathrm{e}^{-11 / 36}$ but will repel branes that are closer. However, the repulsion will not cancel out the attraction from the 3-branes until $\mu$ is exponentially small, $\log \frac{\mu}{r_{\mathrm{s}}} \sim-\frac{1}{\delta_{f}}$, at which point the D7s can no longer be treated as perturbation. Our interpretation is that the presence of the $\overline{\mathrm{D} 3} \mathrm{~s}$ causes the NS-NS forces and R-R forces between D7s to no longer balance and at sufficiently small distances, the repulsive R-R forces dominate. This repulsion may indicate a possible instability of D7-stacks in this background; if one of the D7s in the stacks fluctuates, then the repulsion between the D7s will reinforce the fluctuation and push the D7s apart. However, in order to confirm this effect, we would need to extend our analysis beyond perturbative treatment of the D7 backreaction and to include the backreaction of branes at finite embedding parameter. Moreover, we have imposed by hand that the D7s asymptote to the unperturbed embedding and hence any fluctuation respecting this boundary condition will eventually relax to the initial configuration. In a more complete treatment, the asymptotic boundary condition would be replaced by fluxes in a UV completion of the geometry. Such an effect must also be taken into account to quantify the conditions for stability.

To be more precise about the response of the D7, we should again consider the proper distances. Due to the increased complexity of the geometry we will not report the result here, but they do not qualitatively change the above picture.

\section{Interactions on the conifold}

The previous section explored the behavior of D7s in the presence of both D3-branes and $\overline{\mathrm{D} 3}$-branes. However, the D3- $\overline{\mathrm{D} 3}$ pairs will annihilate into closed strings and so a significant drawback of the construction is the absence of stability. A more realistic scenario is the one suggested in [17] in which $\overline{\mathrm{D} 3} \mathrm{~s}$ are placed at the bottom of a warped geometry sourced by fluxes rather than by integer D3branes. The best known example, and the one explicitly considered in [17], is the Klebanov-Strassler solution which results from the backreaction of fractional D3s in the deformed conifold. However, the KS geometry is a comparatively complicated solution and even more so when the backreaction of $\overline{\mathrm{D} 3} \mathrm{~s}$ are taken into account $[18,29-36]$. Hence we will first consider the case of D3s and $\overline{\mathrm{D} 3} \mathrm{~s}$ located at a conifold singularity, deferring the discussion of the metastable case to the next section.

The conifold ${ }^{12}$ can be embedded into $C^{4}$ by

$$
z^{A} z^{A}=0, \quad z^{A=1,2,3,4} \in C .
$$

It admits a Calabi-Yau metric

$$
\mathrm{d} s_{6}^{2}=-\frac{3}{4 r^{4}} z^{A} \mathrm{~d} \bar{z}^{\bar{A}} \bar{z}^{\bar{B}} \mathrm{~d} z^{B}+\frac{\sqrt{3}}{\sqrt{2} r} \mathrm{~d} z^{A} \mathrm{~d} \bar{z}^{\bar{A}}
$$

in which

$$
z^{A} \bar{z}^{\bar{A}}=\left(\frac{2}{3}\right)^{3 / 2} r^{3} .
$$

\footnotetext{
${ }^{12}$ See [50] for a review of the conifold and related geometries.
} 
The metric takes the form of a cone over the Einstein-Sasaki space $T^{1,1}$

$$
\mathrm{d} s_{6}^{2}=\mathrm{d} r^{2}+r^{2} \mathrm{~d} s_{T^{1,1}}^{2}
$$

$T^{1,1}$ can in turn be written as an $S^{1}$ fibration over the Einstein-Kähler space $S^{2} \times S^{2}$. Indeed, writing

$$
\begin{aligned}
& z^{1}=\left(\frac{2}{27}\right)^{1 / 4} r^{3 / 2} \mathrm{e}^{\mathrm{i} \psi / 2}\left[\cos \left(\frac{\theta^{1}+\theta^{2}}{2}\right) \cos \left(\frac{\phi^{1}+\phi^{2}}{2}\right)+\mathrm{i} \cos \left(\frac{\theta^{1}-\theta^{2}}{2}\right) \sin \left(\frac{\phi^{1}+\phi^{2}}{2}\right)\right] \\
& z^{2}=\left(\frac{2}{27}\right)^{1 / 4} r^{3 / 2} \mathrm{e}^{\mathrm{i} \psi / 2}\left[-\cos \left(\frac{\theta^{1}+\theta^{2}}{2}\right) \sin \left(\frac{\phi^{1}+\phi^{2}}{2}\right)+\mathrm{i} \cos \left(\frac{\theta^{1}-\theta^{2}}{2}\right) \cos \left(\frac{\phi^{1}+\phi^{2}}{2}\right)\right] \\
& z^{3}=\left(\frac{2}{27}\right)^{1 / 4} r^{3 / 2} \mathrm{e}^{\mathrm{i} \psi / 2}\left[-\sin \left(\frac{\theta^{1}+\theta^{2}}{2}\right) \cos \left(\frac{\phi^{1}-\phi^{2}}{2}\right)+\mathrm{i} \sin \left(\frac{\theta^{1}-\theta^{2}}{2}\right) \sin \left(\frac{\phi^{1}-\phi^{2}}{2}\right)\right] \\
& z^{4}=\left(\frac{2}{27}\right)^{1 / 4} r^{3 / 2} \mathrm{e}^{\mathrm{i} \psi / 2}\left[-\sin \left(\frac{\theta^{1}+\theta^{2}}{2}\right) \sin \left(\frac{\phi^{1}-\phi^{2}}{2}\right)-\mathrm{i} \sin \left(\frac{\theta^{1}-\theta^{2}}{2}\right) \cos \left(\frac{\phi^{1}-\phi^{2}}{2}\right)\right]
\end{aligned}
$$

in which $\theta^{i} \in[0, \pi], \phi^{i} \in[0,2 \pi)$, and $\psi \in[0,4 \pi)$, the metric takes the form

$$
\mathrm{d} s_{6}^{2}=\mathrm{d} r^{2}+r^{2}\left(\frac{1}{3} \mathrm{~d} \psi+\mathcal{A}\right)^{2}+r^{2} \mathrm{~d} s_{S^{2} \times S^{2}}^{2}
$$

where

$$
\mathcal{A}=\frac{1}{3} \sum_{i=1}^{2} \cos \theta^{i} \mathrm{~d} \phi^{i}
$$

and

$$
\mathrm{d} s_{S^{2} \times S^{2}}^{2}=\frac{1}{6} \sum_{i=1}^{2}\left[\left(\mathrm{~d} \theta^{i}\right)^{2}+\sin ^{2} \theta^{i}\left(\mathrm{~d} \phi^{i}\right)^{2}\right] .
$$

The Kähler form for the conifold is

$$
J=r \mathrm{~d} r \wedge\left(\frac{1}{3} \mathrm{~d} \psi+\mathcal{A}\right)+r^{2} \mathcal{J}
$$

where

$$
\mathcal{J}=\frac{\sqrt{3} \mathrm{i}}{2 \sqrt{2} r} z^{A} \wedge \bar{z}^{\bar{A}}-\frac{9 \mathrm{i}}{8 r^{4}} \bar{z}^{\bar{A}} \mathrm{~d} z^{A} \wedge z^{B} \mathrm{~d} \bar{z}^{\bar{B}}=-\frac{1}{6} \sum_{i=1}^{2} \sin \theta^{i} \mathrm{~d} \theta^{i} \wedge \mathrm{d} \phi^{i}=\frac{1}{2} \mathrm{~d} \mathcal{A}
$$

is the Kähler form on $S^{2} \times S^{2}$.

Due to the common conical structure, the backreaction of $N+p$ D3 branes with $p \overline{\mathrm{D} 3}$-branes is captured by

$$
\mathrm{d} s_{6}^{2}=\mathrm{e}^{2 B}\left(\mathrm{~d} r^{2}+r^{2} \mathrm{~d} s_{T^{1,1}}^{2}\right)
$$

where $B$, the warp factor, and the $C_{4}$ potential are again given by (3.2). The $p=0$ case is the Klebanov-Witten solution [51] while the finite $p$ case was first considered in [18]. The KlebanovWitten geometry is dual to an $\mathcal{N}=1 \mathrm{SU}(N) \times \mathrm{SU}(N)$ conformal gauge theory summarized by the quiver in figure 3 . 


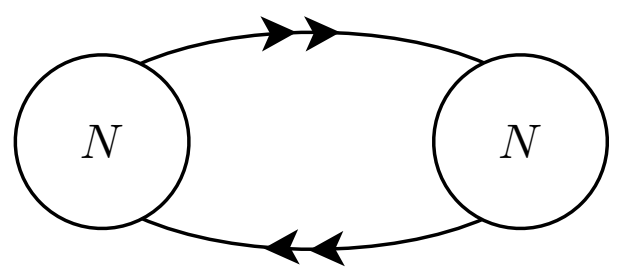

Figure 3. Quiver for the Klebanov-Witten gauge theory.

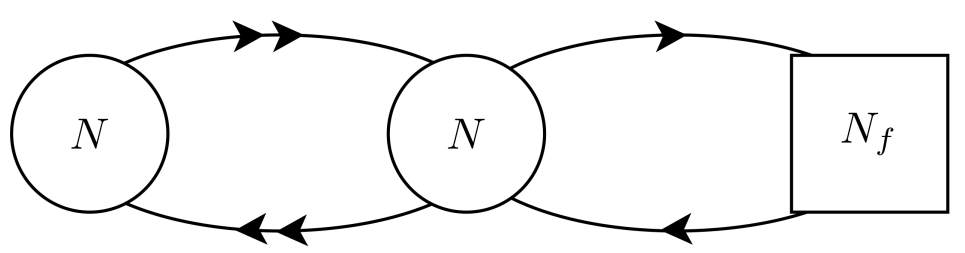

Figure 4. Quiver for the Klebanov-Witten gauge theory flavored by Kuperstein quarks.

\subsection{Kuperstein probes}

Just as in the $A d S^{5} \times S^{5}$ case, a probe D7 brane will be supersymmetric if it is holomorphically embedded into the Calabi-Yau. Here we focus on the Kuperstein embedding [52]

$$
z^{4}=\left(\frac{2}{27}\right)^{1 / 4} \mu
$$

Although our analysis could be extended to Ouyang embeddings [53]

$$
z^{3}+\mathrm{i} z^{4}=\mu
$$

such embeddings are not supersymmetric in the KS geometry without the presence of worldvolume flux $[54,55]$ and so we will not consider them here. Upon adding $N_{f}$ such branes, the dual gauge theory exhibits a $\mathrm{U}\left(N_{f}\right)$ flavor group such that resulting quiver is shown in figure 4.

It is convenient to write the conifold as a foliation of Kuperstein embeddings, similar to what was written in [19]

$$
\begin{aligned}
& z^{1}=\mathrm{i}\left(\frac{2}{27}\right)^{1 / 4} \mu(1+\chi) \mathrm{e}^{\mathrm{i} \zeta}\left[\cos \phi \cosh \left(\frac{t+\mathrm{i} \gamma}{2}\right) \cos \theta-\mathrm{i} \sin \phi \sinh \left(\frac{t+\mathrm{i} \gamma}{2}\right)\right] \\
& z^{2}=\mathrm{i}\left(\frac{2}{27}\right)^{1 / 4} \mu(1+\chi) \mathrm{e}^{\mathrm{i} \zeta}\left[\sin \phi \cosh \left(\frac{t+\mathrm{i} \gamma}{2}\right) \cos \theta+\mathrm{i} \cos \phi \sinh \left(\frac{t+\mathrm{i} \gamma}{2}\right)\right], \\
& z^{3}=\mathrm{i}\left(\frac{2}{27}\right)^{1 / 4} \mu(1+\chi) \mathrm{e}^{\mathrm{i} \zeta} \cosh \left(\frac{t+\mathrm{i} \gamma}{2}\right) \sin \theta, \\
& z^{4}=\left(\frac{2}{27}\right)^{1 / 4} \mu(1+\chi) \mathrm{e}^{\mathrm{i} \zeta}
\end{aligned}
$$


in which $\chi \in[-1, \infty), \zeta \in[0,2 \pi), t \in[0, \infty), \theta \in[0, \pi], \phi \in[0,2 \pi)$, and $\gamma \in[0,4 \pi)$. Our Kuperstein embedding (4.12) is then specified by $\chi=\zeta=0$. The embedding retains an $\mathrm{SO}(3)$ invariance under which $z^{A=1,2,3}$ are rotated among each other. $t$ is an $\mathrm{SO}(3)$-invariant radial-like coordinate satisfying ${ }^{13}$

$$
r=\mu^{2 / 3}(1+\chi)^{2 / 3} \cosh ^{2 / 3} \frac{t}{2} .
$$

It is then useful to define

$$
u=\cosh ^{2 / 3} \frac{t}{2},
$$

so that $u \in[1, \infty)$. We also use the $\mathrm{SO}(3)$ left-invariant 1 -forms [19]

$$
\begin{aligned}
& h^{1}=2\left(\cos \frac{\gamma}{2} \mathrm{~d} \theta-\sin \frac{\gamma}{2} \sin \theta \mathrm{d} \phi\right), \\
& h^{2}=2\left(\sin \frac{\gamma}{2} \mathrm{~d} \theta+\cos \frac{\gamma}{2} \sin \theta \mathrm{d} \phi\right), \\
& h^{3}=\mathrm{d} \gamma-2 \cos \theta \mathrm{d} \phi .
\end{aligned}
$$

In terms of the foliation coordinates, the metric (4.11) takes the form

$$
\begin{aligned}
\mathrm{d} s_{6}^{2}=\mathrm{e}^{2 B} \mu^{4 / 3}(1+\chi)^{4 / 3}\{ & \frac{4 u^{3}-1}{4 u^{3}-4} \mathrm{~d} u^{2}+\frac{u^{2}}{9}\left(1-\frac{1}{4 u^{3}}\right)\left(h^{3}\right)^{2}+\frac{u^{2}}{12}\left(h^{1}\right)^{2}+\frac{u^{2}}{12}\left(1-\frac{1}{u^{3}}\right)\left(h^{2}\right)^{2} \\
& \left.+\frac{4 u}{3(1+\chi)} \mathrm{d} u \mathrm{~d} \chi+\frac{4 u^{2}}{9} \sqrt{1-\frac{1}{u^{3}}} h^{3} \mathrm{~d} \zeta+\frac{4 u^{2}}{9}\left(\frac{\mathrm{d} \chi^{2}}{(1+\chi)^{2}}+\mathrm{d} \zeta^{2}\right)\right\} .
\end{aligned}
$$

The resulting DBI action is then

$$
S_{\mathrm{D} 7}^{\mathrm{DBI}}=-\frac{\tau_{\mathrm{D} 7} \mu^{8 / 3}}{144} \int \mathrm{d}^{4} x \mathrm{~d} u \mathrm{~d}^{3} h \sqrt{W_{0}+W_{1} \chi^{\prime}+W_{2} \chi^{\prime 2}+Y \zeta^{\prime 2}+H_{i j} a_{h^{i}}^{\prime} a_{h^{j}}^{\prime}},
$$

in which

$$
\begin{aligned}
W_{0} & =\left(4 u^{3}-1\right)^{2} \mathrm{e}^{8 B}(1+\chi)^{16 / 3}, \\
W_{1} & =\frac{16 u\left(4 u^{3}-1\right)\left(u^{3}-1\right)}{3} \mathrm{e}^{8 B}(1+\chi)^{13 / 3}, \\
W_{2} & =\frac{16 u^{2}\left(4 u^{3}-1\right)\left(u^{3}-1\right)}{9} \mathrm{e}^{8 B}(1+\chi)^{10 / 3}, \\
Y & =\frac{16}{3} u^{2}\left(u^{3}-1\right) \mathrm{e}^{8 B}(1+\chi)^{16 / 3}, \\
H_{11} & =\frac{\left(2 \pi \alpha^{\prime}\right)^{2}}{\mu^{8 / 3}} \frac{48\left(4 u^{3}-1\right)\left(u^{3}-1\right)}{u^{2}} \mathrm{e}^{4 A+4 B}(1+\chi)^{8 / 3}, \\
H_{22} & =\frac{\left(2 \pi \alpha^{\prime}\right)^{2}}{\mu^{8 / 3}} 48 u^{3}\left(4 u^{3}-1\right) \mathrm{e}^{4 A+4 B}(1+\chi)^{8 / 3}, \\
H_{33} & =\frac{\left(2 \pi \alpha^{\prime}\right)^{2}}{\mu^{8 / 3}} 144 u\left(u^{3}-1\right) \mathrm{e}^{4 A+4 B}(1+\chi)^{8 / 3},
\end{aligned}
$$

with other components of $H_{i j}$ vanishing.

\footnotetext{
${ }^{13}$ The awkward factors of $\left(\frac{2}{27}\right)^{1 / 4}$ appearing in (4.12) and (4.14) were chosen so that this expression has no numerical prefactor.
} 


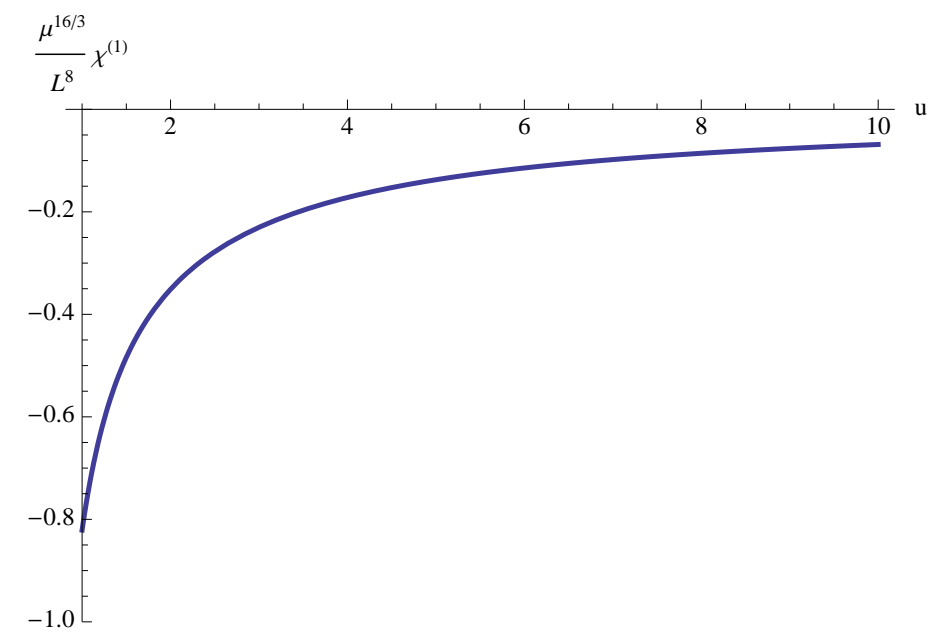

Figure 5. Profile of a would-be Kuperstein D7 probe of $A d S^{5} \times T^{1,1}$ perturbed by $p \ll N$ D3-D3 pairs. The curve is specified by (4.23). Note that the kink at $u=1$ is an artifact of the coordinate system used.

The equations of motion for $\zeta$ and $a_{h^{i}}$ are satisfied by setting $a_{h^{i}}=\zeta=0$. Setting those fields on shell, the action simplifies to

$$
S_{\mathrm{D} 7}=-\frac{\tau_{\mathrm{D} 7} \mu^{8 / 3}}{144} \int \mathrm{d}^{4} x \mathrm{~d} u \mathrm{~d}^{3} h \sqrt{W_{0}+W_{1} \chi^{\prime}+W_{2} \chi^{\prime 2}} .
$$

The resulting equation of motion and boundary conditions again take the forms (3.73) and (3.74) after setting $W_{3}=W_{4}=0$ and replacing the lower bound of the boundary condition with $u=1$. Using that $\chi=0$ is a solution at leading order in $\frac{p}{N}$, the equation of motion at $\mathcal{O}\left(\frac{p}{N}\right)$ is

$$
0=\partial_{u}\left[\frac{u^{2}\left(u^{3}-1\right)}{4 u^{3}-1} \partial_{u} \chi^{(1)}\right]-\frac{6 L^{8}}{5 \mu^{16 / 3}} \frac{1}{u^{8}},
$$

where we have again written $\chi=\sum_{n}\left(\frac{p}{N}\right)^{n} \chi^{(n)}$. The particular solution satisfying the boundary conditions is

$$
\chi^{(1)}=-\frac{3 L^{8}}{700 \mu^{16 / 3}}\left\{\frac{40 u^{7}+60 u^{6}+24 u^{3}-5}{u^{8}}+\frac{240}{\sqrt{3}} \arctan \frac{\sqrt{3}}{1+2 u}\right\} .
$$

This is plotted in figure 5 .

The corresponding minimal radius occurs at $u=1$ and is given by

$$
r_{\min }=\mu^{2 / 3}\left[1-\frac{p}{N} \frac{357+40 \sqrt{3} \pi}{150} \frac{L^{8}}{\mu^{16 / 3}}\right] .
$$

Given that $\mu$ carries different dimensions than the same symbol did in the flat space embedding (3.9), this is qualitatively the same behavior as in the flat space case. Correspondingly, the distance between two such branes and change in energy takes the same qualitative forms as (3.34) and (3.39).

By considering the large $u$ behavior when $p=0$, we can show that $\chi$ is dual to an operator with mass-dimension $\frac{5}{2}$ and again corresponds to a mass term for the dual quarks. $\chi$ is related to a canonically normalized field $\Phi$ such that at large $u, \Phi \sim \chi u^{-3 / 2}$, consistent with the $u^{-1}$ falloff of the perturbed embedding. 


\subsection{Backreacting Kupersteins}

As reviewed in appendix C.2, the backreaction of smeared massless Kupersteins in $A d S^{5} \times T^{1,1}$ takes the same form as the smeared branes in $A d S^{5} \times S^{5}$ does, except that for the case of Kuperstein embeddings, $\delta_{f}=\frac{3 N_{f}}{4 \pi}$. Therefore the forces experienced by probe 3-branes in the presence of backreacting will be the same as in section 3.2. In this section, we will therefore focus on the interaction between Kuperstein branes.

Expressed in terms of the coordinates (4.14), the metric resulting from the backreaction of D7s takes the form

$$
\begin{aligned}
\mathrm{d} s_{6}^{2}=\mathrm{e}^{2 B}\left\{\mathrm{~d} r^{2}+r^{2}\left(\frac{1}{3} \mathrm{~d} \psi+\mathcal{A}\right)^{2}\right\}+\mathrm{e}^{2 C} r^{2} \mathrm{~d} s_{S^{2} \times S^{2}}^{2} \\
=-\frac{3}{4 r^{4}}\left(3 \mathrm{e}^{2 C}-2 \mathrm{e}^{2 B}\right) \bar{z}^{\bar{A}} \mathrm{~d} z^{A} z^{B} \mathrm{~d} \bar{z}^{\bar{B}}+\frac{\sqrt{3}}{\sqrt{2} r} \mathrm{e}^{2 C} \mathrm{~d} z^{A} \mathrm{~d} \bar{z}^{\bar{A}} \\
=\mu^{4 / 3}(1+\chi)^{4 / 3}\left\{\left(\mathrm{e}^{2 B}+\frac{3}{4\left(u^{3}-1\right)} \mathrm{e}^{2 C}\right)\left(\mathrm{d} u^{2}+\frac{u^{3}-1}{9 u}\left(h^{3}\right)^{2}\right)\right. \\
+\frac{u^{2}}{12} \mathrm{e}^{2 C}\left(h^{1}\right)^{2}+\frac{u^{2}}{12}\left(1-\frac{1}{u^{3}}\right) \mathrm{e}^{2 C}\left(h^{2}\right)^{2} \\
+\frac{4 u}{3(1+\chi)} \mathrm{e}^{2 B} \mathrm{~d} u \mathrm{~d} \chi+\frac{4 u^{2}}{9} \sqrt{1-\frac{1}{u^{3}}} \mathrm{e}^{2 B} h^{3} \mathrm{~d} \zeta \\
\left.+\frac{4 u^{2}}{9} \mathrm{e}^{2 B}\left(\frac{\mathrm{d} \chi^{2}}{(1+\chi)^{2}}+\mathrm{d} \zeta^{2}\right)\right\} .
\end{aligned}
$$

Again imposing that the open-string fields depend only on $u$, the induced metric on the 4-cycle is found by making the replacements $\mathrm{d} \chi \rightarrow \chi^{\prime} \mathrm{d} u$ and $\mathrm{d} \zeta \rightarrow \zeta^{\prime} \mathrm{d} u$. The DBI Lagrangian is then

$$
S_{\mathrm{D} 7}^{\mathrm{DBI}}=-\frac{\tau_{\mathrm{D} 7} \mu^{8 / 3}}{144} \int \mathrm{d}^{4} x \mathrm{~d} u \mathrm{~d}^{3} h \sqrt{W_{0}+W_{1} \chi^{\prime}+W_{2} \chi^{\prime 2}+Y \zeta^{\prime 2}+H_{i j} a_{h^{i}}^{\prime} a_{h^{j}}^{\prime}}
$$

in which

$$
\begin{aligned}
W_{0} & =g_{\mathrm{s}}^{-2} \mathrm{e}^{4 C+2 \phi}\left(3 \mathrm{e}^{2 C}+4\left(u^{3}-1\right) \mathrm{e}^{2 B}\right)^{2}(1+\chi)^{16 / 3}, \\
W_{1} & =\frac{16}{3} u\left(u^{3}-1\right) g_{\mathrm{s}}^{-2} \mathrm{e}^{4 C+2 B+2 \phi}\left(3 \mathrm{e}^{2 C}+4\left(u^{3}-1\right) \mathrm{e}^{2 B}\right)(1+\chi)^{13 / 3}, \\
W_{2} & =\frac{16}{9}\left(u^{3}-1\right) u^{2} g_{\mathrm{s}}^{-2} \mathrm{e}^{4 C+2 B+2 \phi}\left(3 \mathrm{e}^{2 C}+4\left(u^{3}-1\right) \mathrm{e}^{2 B}\right)(1+\chi)^{10 / 3}, \\
Y & =\frac{16}{3}\left(u^{3}-1\right) u^{2} g_{\mathrm{s}}^{-2} \mathrm{e}^{6 C+2 B+2 \phi}(1+\chi)^{16 / 3}, \\
H_{11} & =\frac{\left(2 \pi \alpha^{\prime}\right)^{2}}{\mu^{8 / 3}} \frac{48\left(u^{3}-1\right)}{u^{2}} g_{\mathrm{s}}^{-1} \mathrm{e}^{4 A+2 C+\phi}\left(3 \mathrm{e}^{2 C}+4\left(u^{3}-1\right) \mathrm{e}^{2 B}\right)(1+\chi)^{8 / 3}, \\
H_{22} & =\frac{\left(2 \pi \alpha^{\prime}\right)^{2}}{\mu^{8 / 3}} 48 u g_{\mathrm{s}}^{-1} \mathrm{e}^{4 A+2 C+\phi}\left(3 \mathrm{e}^{2 C}+4\left(u^{3}-1\right) \mathrm{e}^{2 B}\right)(1+\chi)^{8 / 3}, \\
H_{33} & =\frac{\left(2 \pi \alpha^{\prime}\right)^{2}}{\mu^{8 / 3}} 144\left(u^{3}-1\right) u g_{\mathrm{s}}^{-1} \mathrm{e}^{4 A+4 C+\phi}(1+\chi)^{8 / 3},
\end{aligned}
$$

with other components of $H_{i j}$ vanishing.

The 8 -form potential again takes the form (3.66). Since $\mathrm{d} \psi$ is closed we can write this as

$$
C_{8}=Q_{f} \frac{r^{3}}{4} g_{\mathrm{s}}^{-2} \mathrm{e}^{2 \phi+4 C} \operatorname{dvol}_{R^{3,1}} \wedge \mathrm{d} r \wedge\left(\frac{1}{3} \mathrm{~d} \psi+\mathcal{A}\right) \wedge \mathcal{J} .
$$


Using

$$
\mathrm{d} r \wedge\left(\frac{1}{3} \mathrm{~d} \psi+\mathcal{A}\right)=\frac{3 \mathrm{i}}{4 r^{5}} \bar{z}^{\bar{A}} \mathrm{~d} z^{A} \wedge z^{B} \mathrm{~d} \bar{z}^{\bar{B}}, \quad \mathcal{J}=\frac{\mathrm{i} \sqrt{3}}{2 \sqrt{2} r^{3}} \mathrm{~d} z^{A} \wedge \mathrm{d} \bar{z}^{\bar{A}}-\frac{9}{8 r^{6}} \bar{z}^{\bar{A}} \mathrm{~d} z^{A} \wedge z^{B} \mathrm{~d} \bar{z}^{\bar{B}}
$$

we have

$$
\begin{aligned}
C_{8}=Q_{f} & \frac{\mu^{8 / 3}(1+\chi)^{2} u^{3}}{4} g_{\mathrm{s}}^{-2} \mathrm{e}^{2 \phi+4 C} \mathrm{dvol}_{R^{3,1}} \\
\wedge & \left\{\frac{(1+\chi)^{2 / 3}}{36}\left[\left(1-\frac{1}{u^{3}}\right) \mathrm{d} u+\frac{2}{3(1+\chi)} \frac{u^{3}-1}{u^{2}} \mathrm{~d} \chi\right] \wedge h^{1} \wedge h^{2} \wedge h^{3}\right. \\
& -\frac{1}{9(1+\chi)^{1 / 3} u^{3 / 2} \sqrt{u^{3}-1}} \mathrm{~d} u \wedge \mathrm{d} \zeta \wedge \mathrm{d} \chi \wedge h^{3} \\
& \left.+\frac{(1+\chi)^{2 / 3}}{18}\left[\sqrt{1-\frac{1}{u^{3}}} \mathrm{~d} u+\frac{2}{3(1+\chi)} \sqrt{\frac{u^{3}-1}{u}} \mathrm{~d} \chi\right] \wedge \mathrm{d} \zeta \wedge h^{1} \wedge h^{2}\right\} .
\end{aligned}
$$

When pulling back to the worldvolume, we make the replacement $\mathrm{d} \chi \rightarrow \chi^{\prime} \mathrm{d} u$ and $\mathrm{d} \zeta \rightarrow \zeta^{\prime} \mathrm{d} u$ and hence

$$
\mathrm{P}\left[C_{8}\right]=Q_{f} \frac{\mu^{8 / 3}(1+\chi)^{8 / 3}}{144} g_{\mathrm{s}}^{-2} \mathrm{e}^{2 \phi+4 C}\left(u^{3}-1\right)\left[1+\frac{2 u \chi^{\prime}}{3(1+\chi)}\right] \operatorname{dvol}_{R^{3,1}} \wedge \mathrm{d} u \wedge h^{1} \wedge h^{2} \wedge h^{3} .
$$

We can again use the calibration condition in the supersymmetric case to determine the orientation. When $p=0$,

$$
\mathrm{dvol}_{\Sigma^{4}}=-\frac{1}{2} \mathrm{P}[J \wedge J] \sim-\mathrm{d} u \wedge h^{1} \wedge h^{2} \wedge h^{3}
$$

and so writing

$$
S_{\mathrm{D} 7}^{\mathrm{CS}}=-\frac{\tau_{\mathrm{D} 7} \mu^{8 / 3}}{144} \int \mathrm{d}^{4} x \mathrm{~d} u \mathrm{~d}^{3} h\left\{W_{3}+W_{4} \chi^{\prime}\right\}
$$

we have

$$
\begin{aligned}
& W_{3}=Q_{f}\left(u^{3}-1\right) g_{\mathrm{s}}^{-1} \mathrm{e}^{2 \phi+4 C}(1+\chi)^{8 / 3}, \\
& W_{4}=\frac{2}{3} Q_{f} u\left(u^{3}-1\right) g_{\mathrm{s}}^{-1} \mathrm{e}^{2 \phi+4 C}(1+\chi)^{5 / 3} .
\end{aligned}
$$

The equations of motion for the gauge field and $\zeta$ are solved by taking $a_{h^{i}}=0$ and $\zeta=0$ while for $\chi$ we again have (3.73) and (3.74). The resulting solution is a little cumbersome, but for large $u$,

$$
\chi \sim-\delta_{p} \frac{24 L^{8}}{35 \mu^{16 / 3} u}-\delta_{p}^{2} \frac{96(88+15 \sqrt{3} \pi-45 \log 3) L^{16}}{6125 \mu^{32 / 3} u}+\delta_{p} \delta_{f} \frac{8 L^{8} \log u}{35 \mu^{16 / 3} u} .
$$

This is again qualitatively the same analytic behavior that we encountered in the $N_{f}=0$ case, aside from the logarithmic running which is a result of the non-trivial RG flow induced by the backreacting flavors. The minimum radius obtained is

$$
r_{\min }=\mu^{2 / 3}\left\{1-\delta_{p} \frac{a L^{8}}{\mu^{16 / 3}}-\delta_{p}^{2} \frac{b L^{16}}{\mu^{32 / 3}}-\delta_{p} \delta_{f} \frac{L^{8}}{\mu^{16 / 3}}\left[c \log \frac{\mu^{2 / 3}}{r_{\mathrm{s}}}+d\right]\right\},
$$

where the coefficients can be expressed precisely but have the approximate values

$$
a \approx 0.547, \quad b \approx 1.90, \quad c \approx 0.730, \quad d \approx 0.961 .
$$

The qualitative behavior is similar to the flat space case: for $\mu \geq r_{\mathrm{s}} \mathrm{e}^{-d / c}$ the backreacting flavors add to the attraction, while for smaller $\mu$, the attraction is reduced. 


\section{$5 \quad$ D7s and anti-D3s in Klebanov-Tseytlin}

As discussed previously, the above examples are unstable in that the spectrum of open strings stretching from a D3-brane to an D3-brane contains a tachyon indicating that the branes will annihilate into closed strings. An alternative and metastable construction is that of [17] in which $\overline{\mathrm{D} 3} \mathrm{~s}$ are added to a geometry warped by the presence of 3-form flux rather than D3-branes

The Klebanov-Tseytlin (KT) solution [56] accomplishes this by considering the presence of fractional D3-branes resulting from $M$ D5s wrapping a collapsing 2-cycle of the conifold. The resulting dual theory is best described in terms of a cascade of self-similar Seiberg dualities where at each stage the quiver takes a form similar to that in figure 3, except at each stage in the cascade the difference in rank between the two nodes is $M$. In terms of the angles appearing in (4.5), it is useful to define the 1 -forms

$$
\begin{aligned}
e^{1} & =-\sin \theta^{1} \mathrm{~d} \varphi^{1}, & & e^{2}=\mathrm{d} \theta^{1}, \\
e^{3} & =\cos \psi \sin \theta^{2} \mathrm{~d} \varphi^{2}-\sin \psi \mathrm{d} \theta^{2}, & & e^{4}=\sin \psi \sin \theta^{2} \mathrm{~d} \varphi^{2}+\cos \psi \mathrm{d} \theta^{2}, \\
e^{5} & =\mathrm{d} \psi+\cos \theta^{1} \mathrm{~d} \varphi^{1}+\cos \theta^{2} \mathrm{~d} \varphi^{2} . & &
\end{aligned}
$$

and then $[57]$

$$
g^{1,3}=\frac{1}{\sqrt{2}}\left(e^{1} \mp e^{3}\right), \quad g^{2,4}=\frac{1}{\sqrt{2}}\left(e^{2} \mp e^{4}\right), \quad g^{5}=e^{5} .
$$

In terms of these forms, the 3 -form flux resulting from the fractional D3s is

$$
F_{3}=\frac{\alpha^{\prime} M}{4} g^{5} \wedge\left(g^{1} \wedge g^{2}+g^{3} \wedge g^{4}\right), \quad H_{3}=\frac{3 \alpha^{\prime} g_{\mathrm{s}} M}{4 r} \mathrm{~d} r \wedge\left(g^{1} \wedge g^{2}+g^{3} \wedge g^{4}\right) .
$$

Such flux is imaginary self dual (ISD) in that $* G_{3}=\mathrm{i} G_{3}$ where $G_{3}=F_{3}-\mathrm{ie}^{-\phi} H_{3}$ and $*$ is the unwarped Hodge- $*$ built from the $6 \mathrm{~d}$ metric

$$
\mathrm{d} s_{6}^{2}=\mathrm{d} r^{2}+\frac{r^{2}}{9}\left(g^{5}\right)^{2}+\frac{r^{2}}{6} \sum_{i=1}^{4}\left(g^{i}\right)^{2} .
$$

Since the background is ISD, we have $\Phi_{-}=0$ and

$$
\mathrm{e}^{-4 A}=\frac{27 \pi \alpha^{\prime} g_{\mathrm{s}}}{4 r^{4}}\left\{N+\frac{3 g_{\mathrm{s}} M^{2}}{2 \pi}\left[\log \frac{r}{r_{0}}+\frac{1}{4}\right]\right\} .
$$

The naked singularity exhibited by this solution is resolved by the deformation of the conifold, the effects of which become important at $r \approx r_{0}$. The resulting supergravity solution is the KlebanovStrassler geometry [16].

The backreaction of $\overline{\mathrm{D} 3} \mathrm{~s}$ on this geometry was first considered in [18] (and is known as the DKM solution). The result is a non-ISD flux and a squashed metric

$$
\begin{aligned}
\mathrm{d} s_{6}^{2} & =\mathrm{e}^{2 B}\left\{\mathrm{~d} r^{2}+\frac{r^{2}}{9}\left(g^{5}\right)^{2}\right\}+\frac{r^{2}}{6} \mathrm{e}^{2 C} \sum_{i=1}^{4}\left(g^{i}\right)^{2}, \\
\mathrm{e}^{2 B} & =1+\frac{3 \alpha^{\prime 2} \mathcal{S}}{4 r^{4}} \\
\mathrm{e}^{2 C} & =1-\frac{\alpha^{\prime 2} \mathcal{S}}{4 r^{4}} \\
F_{3} & =\frac{\alpha^{\prime} M}{4} g^{5} \wedge\left(g^{1} \wedge g^{2}+g^{3} \wedge g^{4}\right), \\
H_{3} & =\alpha^{\prime} \beta^{\prime} \mathrm{d} r \wedge\left(g^{1} \wedge g^{2}+g^{3} \wedge g^{4}\right),
\end{aligned}
$$




$$
\begin{aligned}
\beta(r)= & \frac{3 g_{\mathrm{s}} M}{4} \log \frac{r}{r_{0}}+\frac{3 \pi \alpha^{\prime 2} \mathcal{S}}{8 M r^{4}}\left\{N+\frac{3 g_{\mathrm{s}} M^{2}}{\pi}\left[\log \frac{r}{r_{0}}+\frac{3}{8}\right]\right\}, \\
\omega^{-1}=\mathrm{e}^{-4 A}= & \frac{27 \pi g_{s} \alpha^{\prime 2}}{4 r^{4}}\left\{N+\frac{3 g_{\mathrm{s}} M^{2}}{2 \pi}\left[\log \frac{r}{r_{0}}+\frac{1}{4}\right]\right\} \\
& +\frac{135 \pi g_{\mathrm{s}} \alpha^{\prime 4} \mathcal{S}}{32 r^{8}}\left\{N+\frac{12 g_{\mathrm{s}} M^{2}}{5 \pi}\left[\log \frac{r}{r_{0}}+\frac{13}{12}\right]\right\}, \\
\mathrm{e}^{-\phi}= & \frac{1}{g_{\mathrm{s}}}+\frac{3 \alpha^{\prime 2} \mathcal{S}}{g_{\mathrm{s}} r^{4}} \log \frac{r}{r_{0}},
\end{aligned}
$$

in which $\mathcal{S} \sim \frac{p}{N} \mathrm{e}^{-8 \pi N / 3 g_{\mathrm{s}} M^{2}} \frac{r_{0}^{4}}{\alpha^{\prime 2}}$ and we have again written the metric as (4.25). Note that the form of our solution differs from the original DKM solution in [18] by an $\mathcal{O}(1)$ redefinition of $\mathcal{S}$ and a redefinition of the radial coordinate ${ }^{14}$. As emphasized in $[18,30,33]$, the DKM solution does not entirely capture the backreaction of an $\overline{\mathrm{D} 3}$-brane. The most obvious deficiency is that $\Phi_{-}=0$, while it is precisely in the $\Phi_{-}$equation of motion (2.7) that explicit $\overline{\mathrm{D} 3}$-branes appear. However, the consequences of a non-zero $\Phi_{-}$fall off faster than the DKM solution for large $r$ (see (3.2)). Less obvious is the fact that the backreaction of $\overline{\mathrm{D} 3} \mathrm{~s}$ in the full KS solution have modes that drop off more slowly than those appearing in DKM [30-36] but disappear as we take the deformation of the conifold to vanish. For these reasons we will consider the analysis of a Kuperstein brane in DKM as a warm-up to a more complete analysis in future work, anticipating that much of the behavior will be qualitatively captured by our analysis in DKM. Note that in order to trust the DKM solution, we must ensure that $r \gg r_{0}$ and $r \gg \mathcal{S}^{1 / 4} \sqrt{\alpha^{\prime}}$.

Consider a Kuperstein probe of the geometry. We can write the NS-NS potential as (see, e.g. [59])

$$
B_{2}=\alpha^{\prime} \beta\left(g^{1} \wedge g^{2}+g^{3} \wedge g^{4}\right)=\frac{27 \mathrm{i} \alpha^{\prime} \beta}{4 r^{6}} \epsilon_{A B C D} z^{A} \bar{z}^{\bar{B}} \mathrm{~d} z^{C} \wedge \mathrm{d} \bar{z}^{\bar{D}}
$$

where $\epsilon_{1234}=+1$. In the $\mathcal{S}=0$ case, the $\mathrm{D} 7$ is holomorphically embedded into the conifold and so $\mathcal{F}_{2}=\mathrm{P}\left[B_{2}\right]+2 \pi \alpha^{\prime} f_{2}$ is $(1,1)$ when $f_{2}=0$. Furthermore one can show [52] that $\mathcal{F}_{2}$ is primitive in the sense that $\mathrm{P}[J] \wedge \mathcal{F}_{2}=0$ where $J$ is the Kähler form of the conifold. Hence, a Kuperstein embedding is supersymmetric in $\mathrm{KT}^{15}$.

Consider a would-be Kuperstein probe of the DKM geometry. Note that again we must take $\mu$ to be sufficiently large to trust the perturbative treatment of the $\overline{\mathrm{D} 3} \mathrm{~s}$ as well treat the deformation of the conifold singularity as a negligible modification of the geometry. The presence of 3-form flux modifies the DBI and CS actions from what was considered in previous sections. In terms of the coordinates (4.14), the NS-NS 2-form takes the form

$$
B_{2}=-\frac{\alpha^{\prime} \beta}{2 u^{5 / 2}}\left\{3 u \mathrm{~d} u \wedge h^{2}+h^{1} \wedge h^{3}\right\} .
$$

The DBI action then takes the form

$$
S_{\mathrm{D} 7}^{\mathrm{DBI}}=-\frac{\tau_{\mathrm{D} 7} \mu^{8 / 3}}{144} \int \mathrm{d}^{4} x \mathrm{~d} u \mathrm{~d}^{3} h \sqrt{W_{0}+W_{1} \chi^{\prime}+W_{2} \chi^{\prime 2}+Y \zeta^{\prime 2}+Y_{i} \zeta^{\prime} a_{h^{i}}^{\prime}+H_{i} a_{h^{i}}^{\prime}+H_{i j} a_{h^{i}}^{\prime} a_{h^{j}}^{\prime}},
$$

\footnotetext{
${ }^{14}$ It was pointed out in [19] that the DKM solution, as originally expressed in [18], exhibits a non-Hermitian metric when expressed in terms of the unperturbed complex structure (i.e. the solution of [18] has $g_{z z} \neq 0$ ). This feature is not exhibited by (4.25) due to the different ansatz used. This should not be seen as contrary to the expectation that non-supersymmetric sources will generically induce such non-Hermitian elements (see, e.g., the discussion in [58]) as the coordinate redefinition that brings the solution [18] to the form (4.25) is non-holomorphic.

${ }^{15}$ In contrast, while $\mathrm{P}\left[B_{2}\right]$ is course still $(1,1)$ for an Ouyang embedding, it is not primitive, and hence, as mentioned earlier, an Ouyang embedding must be magnetized in order to be supersymmetric [54, 55].
} 
in which

$$
\begin{aligned}
W_{0}= & g_{\mathrm{s}}^{-2} \mathrm{e}^{2 \phi}\left[(1+\chi)^{8 / 3} \mathrm{e}^{2 C}\left(3 \mathrm{e}^{2 C}+4\left(u^{3}-1\right) \mathrm{e}^{2 B}\right)+\frac{27 \alpha^{\prime 2}}{\mu^{8 / 3}} \frac{\beta^{2}}{u^{4}} g_{\mathrm{s}} \mathrm{e}^{4 A-\phi}\right]^{2}, \\
W_{1}= & \frac{16}{3} g_{\mathrm{s}}^{-2} \mathrm{e}^{2 \phi}(1+\chi)^{13 / 3} \mathrm{e}^{4 C+2 B} u\left(u^{3}-1\right)\left(3 \mathrm{e}^{2 C}+4\left(u^{3}-1\right) \mathrm{e}^{2 B}\right) \\
& +\frac{144 \alpha^{\prime 2}}{\mu^{8 / 3}}(1+\chi)^{5 / 3} \mathrm{e}^{2 B+2 C} \beta^{2}\left(1-\frac{1}{u^{3}}\right) g_{\mathrm{s}}^{-1} \mathrm{e}^{4 A+\phi}, \\
W_{2}= & \frac{16}{9} g_{\mathrm{s}}^{-2} \mathrm{e}^{2 \phi}(1+\chi)^{10 / 3} \mathrm{e}^{4 C+2 B} u^{2}\left(u^{3}-1\right)\left(3 \mathrm{e}^{2 C}+4\left(u^{3}-1\right) \mathrm{e}^{2 B}\right) \\
& +\frac{48 \alpha^{\prime 2}}{\mu^{8 / 3}}(1+\chi)^{2 / 3}\left(u-\frac{1}{u^{2}}\right) \mathrm{e}^{2 B+2 C} \beta^{2} g_{\mathrm{s}}^{-1} \mathrm{e}^{4 A+\phi}, \\
Y= & \frac{16}{3} g_{\mathrm{s}}^{-2} \mathrm{e}^{2 \phi}(1+\chi)^{16 / 3} \mathrm{e}^{6 C+2 B}\left(u^{3}-1\right) u^{2} \\
& +\frac{48 \alpha^{\prime 2}}{\mu^{8 / 3}}(1+\chi)^{8 / 3}\left(u-\frac{1}{u^{2}}\right) \mathrm{e}^{2 B+2 C} \beta^{2} g_{\mathrm{s}}^{-1} \mathrm{e}^{4 A+\phi}, \\
Y_{1}= & -\frac{192\left(2 \pi \alpha^{\prime}\right) \alpha^{\prime}}{\mu^{8 / 3}}(1+\chi)^{8 / 3} \frac{\left(u^{3}-1\right)^{3 / 2}}{u^{2}} \mathrm{e}^{2 B+2 C} \beta g_{\mathrm{s}}^{-1} \mathrm{e}^{4 A+\phi}, \\
H_{2}= & -\frac{72\left(2 \pi \alpha^{\prime}\right) \alpha^{\prime}}{\mu^{8 / 3} u^{3 / 2}} \mathrm{e}^{4 A} \beta\left[(1+\chi)^{8 / 3}\left(3 \mathrm{e}^{2 C}+4\left(u^{3}-1\right) \mathrm{e}^{2 B}\right) g_{\mathrm{s}}^{-1} \mathrm{e}^{\phi}+\frac{27 \alpha^{\prime 2}}{\mu^{8 / 3} u^{4}} \beta^{2} \mathrm{e}^{4 A}\right], \\
H_{11}= & \frac{48\left(2 \pi \alpha^{\prime}\right)^{2}}{\mu^{8 / 3}}(1+\chi)^{8 / 3} \frac{\left(u^{3}-1\right)}{u^{2}}\left(3 \mathrm{e}^{2 C}+4\left(u^{3}-1\right) \mathrm{e}^{2 B}\right) g_{\mathrm{s}}^{-1} \mathrm{e}^{4 A+\phi}, \\
H_{22}= & \frac{48\left(2 \pi \alpha^{\prime}\right)^{2}}{\mu^{8 / 3}}\left[(1+\chi)^{8 / 3} u \mathrm{e}^{2 C}\left(3 \mathrm{e}^{2 C}+4\left(u^{3}-1\right) \mathrm{e}^{2 B}\right) g_{\mathrm{s}}^{-1} \mathrm{e}^{4 A+\phi}+\frac{27 \alpha^{\prime 2}}{\mu^{8 / 3}} \frac{\beta^{2}}{u^{3}} \mathrm{e}^{8 A}\right], \\
H_{33}= & \frac{144\left(2 \pi \alpha^{\prime}\right)^{2}}{\mu^{8 / 3}}(1+\chi)^{8 / 3}\left(u^{3}-1\right) u g_{\mathrm{s}}^{-1} \mathrm{e}^{4 A+4 C+\phi},
\end{aligned}
$$

with the remaining $H_{i j}, H_{i}$, and $Y_{i}$ vanishing.

The Chern-Simons action receives a contribution from the 4 -form potential

$$
S_{\mathrm{D} 7}^{\mathrm{CS}(4)}=\frac{1}{2} \tau_{\mathrm{D} 7} g_{\mathrm{s}} \int \mathrm{P}\left[C_{4}\right] \wedge \mathcal{F}_{2} \wedge \mathcal{F}_{2} .
$$

Using that the pullback of $B_{2}$ is trivial and taking the orientation defined by (4.32), this contribution becomes

$$
\begin{aligned}
S_{\mathrm{D} 7}^{\mathrm{CS}(4)} & =\frac{\tau_{\mathrm{D} 7}}{8} \int \mathrm{d}^{8} \xi \omega \epsilon^{a b c d} \mathcal{F}_{a b} \mathcal{F}_{c d} \\
& =\frac{\tau_{\mathrm{D} 7} \mu^{8 / 3}}{144} \int \mathrm{d}^{8} \xi \omega\left\{\frac{27 \alpha^{\prime 2}}{\mu^{8 / 3}} \frac{\beta^{2}}{u^{4}}-\frac{36\left(2 \pi \alpha^{\prime}\right) \alpha^{\prime}}{\mu^{8 / 3}} \frac{\beta}{u^{3 / 2}} a_{h^{2}}^{\prime}\right\} .
\end{aligned}
$$

in which $\epsilon^{u 123}=-1$.

In addition, there is a contribution from $C_{6}$, the magnetic dual of $C_{2}$,

$$
S_{\mathrm{D} 7}^{\mathrm{CS}(6)}=\tau_{\mathrm{D} 7} g_{\mathrm{s}} \int \mathrm{P}\left[C_{6}\right] \wedge \mathcal{F}_{2} .
$$

The potential $C_{6}$ is such that

$$
F_{7}=\mathrm{d} C_{6}-H_{3} \wedge C_{4}=-g_{\mathrm{s}}^{-1} \mathrm{e}^{\phi} \hat{*} F_{3},
$$


where $\hat{*}$ is the Hodge- $*$ built from the $10 \mathrm{~d}$ Einstein-frame metric. Since $\iota_{\partial_{\mu}} F_{3}=0$, we have

$$
\hat{*} F_{3}=\mathrm{e}^{4 A} \mathrm{dvol}_{R^{3,1}} \wedge * F_{3},
$$

in which $*$ is the Hodge- $*$ built from the unwarped $6 \mathrm{~d}$ metric $g_{m p}$. Hence,

$$
\mathrm{d} C_{6}=g_{\mathrm{s}}^{-1} \mathrm{e}^{\phi} \operatorname{dvol}_{R^{3,1}} \wedge\left(\omega \mathrm{e}^{-\phi} H_{3}-\mathrm{e}^{4 A} * F_{3}\right) .
$$

It is easy to check that in an ISD background, $\mathrm{d} C_{6}$ vanishes and so we can take $C_{6}$ to vanish. In DKM this becomes

$$
\mathrm{d} C_{6}=\alpha^{\prime} g_{\mathrm{s}}^{-1} \mathrm{e}^{4 A}\left(\frac{\mathrm{d} \beta}{\mathrm{d} r}-\mathrm{e}^{\phi} \frac{3 M}{4 r}\right) \mathrm{d} r \wedge\left(g^{1} \wedge g^{2}+g^{3} \wedge g^{4}\right) .
$$

Writing

$$
C_{6}=g_{\mathrm{s}}^{-1} \operatorname{dvol}_{R^{3,1}} \wedge \tilde{C}_{2}, \quad \tilde{C}_{2}=\alpha^{\prime} \gamma\left(g^{1} \wedge g^{2}+g^{3} \wedge g^{4}\right),
$$

we have

$$
\gamma=-\frac{2 \mathcal{S}}{9 g_{\mathrm{s}} M} \log \frac{r}{r_{0}}
$$

Note that $\tilde{C}_{2}$ is parallel to $B_{2}$.

$$
\begin{aligned}
S_{\mathrm{D} 7}^{\mathrm{CS}(6)} & =\frac{\tau_{\mathrm{D} 7} g_{\mathrm{s}}}{4} \int \mathrm{d}^{8} \xi \epsilon^{a b c d} \tilde{C}_{a b} \mathcal{F}_{c d} \\
& =\frac{\tau_{\mathrm{D} 7} \mu^{8 / 3}}{144} \int \mathrm{d}^{8} x\left\{\frac{54 \alpha^{\prime 2}}{\mu^{8 / 3}} \frac{\beta \gamma}{u^{4}}-\frac{36\left(2 \pi \alpha^{\prime}\right) \alpha^{\prime}}{\mu^{8 / 3}} \frac{\gamma}{u^{3 / 2}} a_{h^{2}}^{\prime}\right\} .
\end{aligned}
$$

The DKM solution has $C_{0}=0$ and hence there is no $C_{0}$ or $C_{8}$ contribution to the Chern-Simons action. Finally, with the assumption that $f_{\mu \nu}=0$, neither $C_{2}$ nor the magnetic part of $C_{4}$ contribute. Hence we can write the total CS action as

$$
S_{\mathrm{D} 7}^{\mathrm{CS}}=-\frac{\tau_{\mathrm{D} 7} \mu^{8 / 3}}{144} \int \mathrm{d}^{8} \xi\left\{W_{3}+K_{i} a_{h^{i}}^{\prime}\right\},
$$

with

$$
\begin{aligned}
& W_{3}=-\frac{27 \alpha^{\prime 2}}{\mu^{8 / 3}} \frac{\beta}{u^{4}}(\omega \beta+2 \gamma), \\
& K_{2}=\frac{36\left(2 \pi \alpha^{\prime}\right) \alpha^{\prime}}{\mu^{8 / 3}} \frac{\omega \beta+\gamma}{u^{3 / 2}} .
\end{aligned}
$$

The functions $W_{i}, Y, Y_{i}, H_{i}, H_{i j}$ and $K_{i}$ depend only on $u$ and $\chi$. Hence $\zeta, a_{h^{1}}$ and $a_{h^{3}}$ only appear in the action through the derivatives and furthermore only in the combinations $\zeta^{\prime} a_{h^{1}}^{\prime}, \zeta^{\prime 2}, a_{h^{1}}^{\prime 2}$ and $a_{h^{3}}^{\prime 2}$. Therefore the equations of motion for those fields are solved by taking $\zeta=a_{h^{1}}=a_{h^{3}}=0$. However, due to the non-trivial NS-NS potential, we cannot also take $a_{h^{2}}=0$. The remaining equations of motion are

$$
\begin{aligned}
0= & \partial_{u}\left[\frac{W_{2}}{\sqrt{W}} \partial_{u} \chi\right]-\frac{1}{2} \frac{\partial_{\chi} W_{2}}{\sqrt{W}} \chi^{\prime 2}-\frac{1}{2} \frac{\partial_{\chi} H_{22}}{\sqrt{W}} a_{h^{2}}^{\prime 2}-\frac{1}{2} \frac{\partial_{\chi} W_{1}}{\sqrt{W}} \chi^{\prime} \\
& -\frac{1}{2} \frac{\partial_{\chi} H_{2}}{\sqrt{W}} a_{h^{2}}^{\prime}-\partial_{\chi} K_{2} a_{h^{2}}^{\prime}+\frac{1}{2} \partial_{u}\left[\frac{W_{1}}{\sqrt{W}}\right]-\frac{1}{2} \frac{\partial_{\chi} W_{0}}{\sqrt{W}}-\partial_{\chi} W_{3}, \\
0= & \partial_{u}\left[\frac{H_{22}}{\sqrt{W}} \partial_{u} a_{h^{2}}\right]+\frac{1}{2} \partial_{u}\left[\frac{H_{2}}{\sqrt{W}}\right]+\partial_{u} K_{2},
\end{aligned}
$$


in which

$$
W=W_{0}+W_{1} \chi^{\prime}+W_{2} \chi^{\prime 2}+H_{2} a_{h^{i}}^{\prime}+H_{22} a_{h^{2}}^{\prime 2} .
$$

The boundary terms vanish by imposing Dirichlet boundary conditions $\chi \rightarrow 0$ and $a_{h^{2}} \rightarrow 0$ as $u \rightarrow \infty$, while at $u=1$ we impose the Neumann conditions

$$
\begin{aligned}
& 0=\frac{W_{2}}{\sqrt{W}} \chi^{\prime}+\frac{W_{1}}{2 \sqrt{W}}, \\
& 0=\frac{H_{22}}{\sqrt{W}} a_{h^{2}}^{\prime}+\frac{H_{2}}{2 \sqrt{W}}+K_{2} .
\end{aligned}
$$

Performing the expansions

$$
\chi=\sum_{n=0}^{\infty} \mathcal{S}^{n} \chi^{(n)}, \quad a_{h^{2}}=\sum_{n=0}^{\infty} \mathcal{S}^{n} a^{(n)},
$$

the $\mathcal{O}\left(\mathcal{S}^{0}\right)$ contributions to the equations are satisfied by taking $\chi^{(0)}=0$ and $a^{(0)}=0$. The $\mathcal{O}(\mathcal{S})$ contributions to the equations take the form

$$
\begin{aligned}
& 0=\partial_{u}\left[\mathcal{K}_{\chi \chi} \partial_{u} \chi^{(1)}\right]+\partial_{u}\left[\mathcal{K}_{a \chi} \partial_{u} a^{(1)}\right]+t_{\chi}, \\
& 0=\partial_{u}\left[\mathcal{K}_{a a} \partial_{u} a^{(1)}\right]+\partial_{u}\left[\mathcal{K}_{a \chi} \partial_{u} \chi^{(1)}\right]+t_{a},
\end{aligned}
$$

in which

$$
\begin{aligned}
& \mathcal{K}_{\chi \chi}=\frac{16 u^{2}\left(u^{3}-1\right)\left(8 \pi N+3 g_{\mathrm{s}} M^{2}+12 g_{\mathrm{s}} M^{2} \log \frac{u \mu^{2 / 3}}{r_{0}}+6 g_{\mathrm{s}} M^{2} \log ^{2} \frac{u \mu^{2 / 3}}{r_{0}}\right)}{54 g_{\mathrm{s}} M^{2} \log ^{2} \frac{u \mu^{2 / 3}}{r_{0}}+3\left(4 u^{3}-1\right)\left(8 \pi N+3 g_{\mathrm{s}} M^{2}+12 g_{\mathrm{s}} M^{2} \log \frac{u \mu^{2 / 3}}{r_{0}}\right)}, \\
& \mathcal{K}_{a a}=\frac{2048 \pi^{2} u^{5}\left(4 u^{3}-1\right)}{3 g_{\mathrm{s}}\left[54 g_{\mathrm{s}} M^{2} \log ^{2} \frac{u \mu^{2 / 3}}{r_{0}}+3\left(4 u^{3}-1\right)\left(8 \pi N+3 g_{\mathrm{s}} M^{2}+12 g_{\mathrm{s}} M^{2} \log \frac{u \mu^{2 / 3}}{r_{0}}\right)\right]}, \\
& \mathcal{K}_{a \chi}=\frac{512 M \pi u^{7 / 2}\left(u^{3}-1\right) \log \frac{u \mu^{2 / 3}}{r_{0}}}{54 g_{\mathrm{s}} M^{2} \log ^{2} \frac{u \mu^{2 / 3}}{r_{0}}+3\left(4 u^{3}-1\right)\left(8 \pi N+3 g_{\mathrm{s}} M^{2}+12 g_{\mathrm{s}} M^{2} \log \frac{u \mu^{2 / 3}}{r_{0}}\right)},
\end{aligned}
$$

and

$$
\begin{aligned}
t_{\chi} & =-\frac{2 \alpha^{\prime 2}}{\mu^{8 / 3}}\left(18 \log \frac{u \mu^{2 / 3}}{r_{0}}-5\right), \\
t_{a} & =\frac{8 \pi \alpha^{\prime 2}}{g_{\mathrm{s}} M u^{5 / 2} \mu^{8 / 3}}\left(3 \log \frac{u \mu^{2 / 3}}{r_{0}}-2\right) .
\end{aligned}
$$

The solutions to these equations are

$$
\begin{aligned}
& \chi^{(1)}=c_{1}-\int \mathrm{d} u \frac{1}{\mathcal{K}_{\chi \chi} \mathcal{K}_{a a}-\mathcal{K}_{a \chi}^{2}}\left\{\mathcal{K}_{a a}\left(\int \mathrm{d} u t_{\chi}+c_{3}\right)-\mathcal{K}_{a \chi}\left(\int \mathrm{d} u t_{a}+c_{4}\right)\right\}, \\
& a^{(1)}=c_{2}+\int \mathrm{d} u \frac{1}{\mathcal{K}_{\chi \chi} \mathcal{K}_{a a}-\mathcal{K}_{a \chi}^{2}}\left\{\mathcal{K}_{a \chi}\left(\int \mathrm{d} u t_{\chi}+c_{3}\right)-\mathcal{K}_{\chi \chi}\left(\int \mathrm{d} u t_{a}+c_{4}\right)\right\} .
\end{aligned}
$$


Imposing the boundary conditions, we have

$$
\begin{aligned}
& \chi^{(1)}=-\frac{9 \alpha^{\prime 2}}{4 \mu^{8 / 3}} \int_{\infty}^{u} \frac{\mathrm{d} x}{x^{5}}\left\{\log ^{2} \frac{x \mu^{2 / 3}}{r_{0}}-x^{3 / 2} \log \frac{x \mu^{2 / 3}}{r_{0}} \log \frac{\mu^{2 / 3}}{r_{0}}\right. \\
& \left.+\frac{4 x^{3}-1}{x^{3}-1}\left[\log \frac{x \mu^{2 / 3}}{r_{0}}-x^{3} \log \frac{\mu^{2 / 3}}{r_{0}}\right]-\frac{4 x^{3}-1}{18}\right\}, \\
& a^{(1)}=\frac{27 \alpha^{\prime 2} g_{\mathrm{s}} M}{64 \pi \mu^{8 / 3}} \int_{\infty}^{u} \frac{\mathrm{d} x}{x^{13 / 2}}\left\{\log ^{3} \frac{x \mu^{2 / 3}}{r_{0}}-x^{3 / 2} \log ^{2} \frac{x \mu^{2 / 3}}{r_{0}} \log \frac{\mu^{2 / 3}}{r_{0}}\right. \\
& +6 \log ^{2} \frac{x \mu^{2 / 3}}{r_{0}}-2 x^{3 / 2}\left(1+2 x^{3 / 2}\right) \log \frac{x \mu^{2 / 3}}{r_{0}} \log \frac{\mu^{2 / 3}}{r_{0}} \\
& -\frac{2}{9} x^{3} \log \frac{x \mu^{2 / 3}}{r_{0}}+\frac{1}{18}\left(\frac{24 \pi N}{g_{\mathrm{s}} M^{2}}+13\right) \log \frac{x \mu^{2 / 3}}{r_{0}} \\
& \left.-\frac{1}{6}\left(\frac{8 \pi N}{g_{\mathrm{s}} M^{2}}+3\right) x^{3 / 2} \log \frac{\mu^{2 / 3}}{r_{0}}\right\} \text {. }
\end{aligned}
$$

The resulting profile and magnetization are plotted for a few different values of $\mu / r_{0}^{3 / 2}$ in figure 6 . There is a qualitative change in the behavior, marked by an apparent repulsion of the probe $\mathrm{D} 7$, as $\mu$ crosses $r_{0}$. Indeed the value of $\chi^{(1)}$ at $u=1$, which is the point of closest approach when $\mathcal{S}=0$, is

$$
\chi^{\mathrm{m}}:=\chi^{(1)}(u=1)=-\frac{3 \alpha^{\prime 2}}{3200 \mu^{8 / 3}}\left\{7475-800 \psi_{1}\left(\frac{1}{3}\right)+9084 \log \frac{\mu^{2 / 3}}{r_{0}}+360 \log ^{2} \frac{\mu^{2 / 3}}{r_{0}}\right\}
$$

in which $\psi_{1}$ is the trigamma function. This is plotted in figure 7 and indeed it changes sign at around $\mu^{2 / 3} \approx r_{0}$, reflecting the fact that $\log \frac{\mu^{2 / 3}}{r_{0}}$ changes sign there. However, as $r_{0}$ is the position at which the effects of a finite deformation of the conifold are expected to become important, the solution is not applicable in that region. We also note that the deformation of the worldvolume falls off more slowly this case than it did in the KW case discussed in section 4.1 where $\delta \chi \sim \mu^{-16 / 3}$. This is a consequence of the 3-form flux which even in supersymmetric configurations gives rise to potentials for 7-brane deformation moduli and induces positive D3-charge on the D7s. Moreover, again as a consequence of the flux, the influence of the $\overline{\mathrm{D} 3}$-branes falls off more slowly than it did in $A d S^{5} \times X^{5}$.

We now consider, as we did in section 3.1, two Kuperstein embeddings. The minimum radii reached by the branes are (assuming $\mu_{i}$ is sufficiently large)

$$
r_{i}=\mu_{i}^{2 / 3}\left(1+\frac{2 \mathcal{S}}{3} \chi_{i}^{\mathrm{m}}\right) .
$$

As argued earlier, the unwarped distance is more natural characterization of the effects of the addition of $\overline{\mathrm{D} 3} \mathrm{~s}$ and we have

$$
\Delta s=\mu_{2}^{2 / 3}-\mu_{1}^{2 / 3}+\frac{\mathcal{S}}{8} \frac{\alpha^{2}\left(\mu_{2}^{2}-\mu_{1}^{2}\right)}{\mu_{2}^{4} \mu_{1}^{4}}+\frac{2}{3}\left(\chi_{2}^{\mathrm{m}} \mu_{2}^{2 / 3}-\chi_{1}^{\mathrm{m}} \mu_{1}^{2 / 3}\right) .
$$

For $\mu_{1} \gtrsim 2 r_{0}^{3 / 2}$, this is positive. Thus, where the solution can be trusted, the separation between the probe D7 increases, as is consistent with the interpretation that the flavor branes fall toward the $\overline{\mathrm{D} 3} \mathrm{~s}$. Note that unlike the conformal $A d S^{5} \times X^{5}$ cases, the Klebanov-Strassler theory has a mass scale at which the theory becomes confined. In principle, we could compare against the motion of the branes against this scale. However, to reliably do so would require studying the motion of D7-branes in the backreaction of $\overline{\mathrm{D} 3} \mathrm{~s}$ in the full KS geometry. Although this solution is known [30-36], the analysis is considerably more involved and we will not consider it here. 


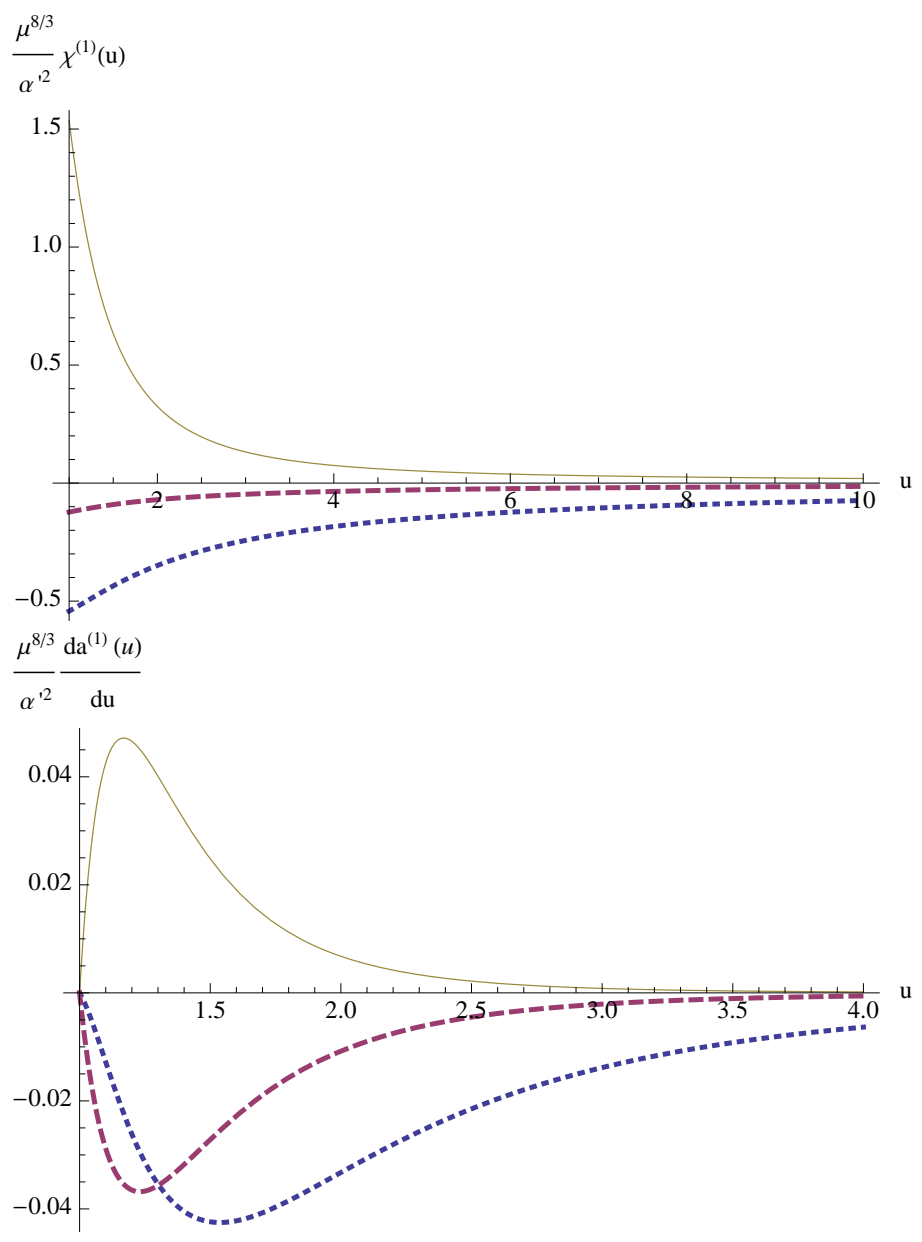

Figure 6. The behavior of a would-be Kuperstein brane probing the DKM geometry (5.6). Top. Deformation of the worldvolume given by (5.31a). The dotted blue line is $\mu=2 r_{0}^{3 / 2}$, the dashed purple line is $\mu=5 r_{0}^{3 / 2}$ and the solid mustard line is $\mu=.9 r_{0}^{3 / 2}$. Bottom. The magnetization $F_{u h^{2}}$, plotted with the same values of $\mu$ and setting $g_{\mathrm{s}}=10^{-6}, M=10, N=1000$. Note that our analysis is only valid for $\mu \gg r_{0}^{3 / 2}$ and the plotted solutions do not satisfy this condition. However, solutions with large $\mu$ do not qualitatively change with respect to the $\mu=2 r_{0}^{3 / 2}$ and $\mu=5 r_{0}^{3 / 2}$ solutions.

Finally, we consider the energy of a Kuperstein brane. As discussed previously, the DKM solution only contains some information about the backreaction of the $\overline{\mathrm{D} 3}$ s. The $\mathcal{O}\left(\mathcal{S}^{2}\right)$ terms may be subdominant compared to these omitted corrections and so we will not include them in our consideration. Therefore, we can only reliably find the energy of the configuration to linear order in $\mathcal{S}$ which does not make use of the perturbed profile. We find however, that even to this order the change in energy is divergent. In particular if the worldvolume is cutoff at $u_{\infty}$,

$$
\Delta \mathcal{E}=\mathcal{E}_{p \neq 0}-\mathcal{E}_{p=0} \sim-\frac{\alpha^{\prime 2} \mathcal{S}}{\mu^{8 / 3}} \log ^{2} u_{\infty}
$$




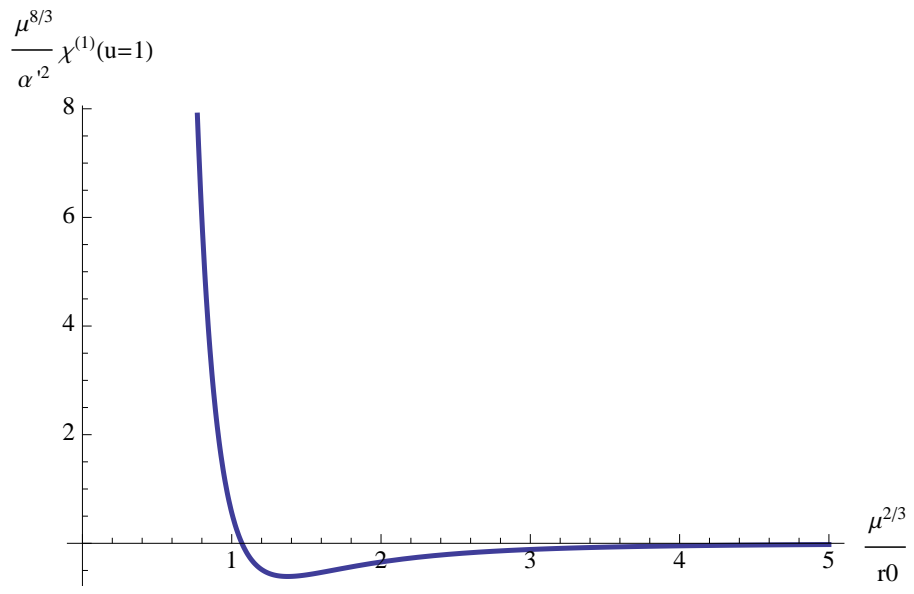

Figure 7. The minimum value of $\chi$ reached by a probe Kuperstein in the DKM solution. Negative values of $\chi$ indicate that the brane is pulled into the center of warping.

\section{Model building with falling flavors}

In the previous section we quantified the extent to which D7-branes probing supersymmetric warped geometries are deformed by the inclusion of $\overline{\mathrm{D} 3}$-branes. The general result was that the worldvolumes of such D7-branes bend toward the $\overline{\mathrm{D} 3}$-branes. In this section we discuss what implications this might have on model building with such 7-branes. We will limit ourselves to a qualitative discussion, leaving a more quantitative treatment for future work. Much of what is presented here is well known in the literature and so this section serves primarily to place the effects that we have discussed here into a wider context by pointing out where the deformation of the D7s may have substantial impact.

One immediate consequence is the correction to the soft terms resulting from dimensional reduction of the D7 worldvolume theory ${ }^{16}$. For example, the $4 \mathrm{~d}$ gaugino resulting from such a dimensional reduction receives a mass from non-supersymmetric fluxes that is given by an integral over the 4cycle [60-63] wrapped by the D7 that schematically takes the form

$$
m_{1 / 2} \sim \int_{\Sigma^{4}} \operatorname{dvol}_{\Sigma^{4}} \eta^{\dagger} \mathrm{P}\left[\gamma^{m n p} G_{m n p}\right] \eta \sim \int_{\Sigma^{4}} \operatorname{dvol}_{\Sigma^{4}} \bar{\Omega} \cdot G_{3},
$$

in which $\eta$ is the internal part of the gaugino wavefunction (which to leading order in the nonsupersymmetric perturbation is proportional to the Killing spinor of the underlying Calabi-Yau), $\Omega$ is the fundamental 3-form of the Calabi-Yau, and we have omitted contributions from the worldvolume flux. This term describes a non-vanishing gaugino mass resulting from a $(3,0)$ flux. However, the calculation of the pullback in (6.1) assumes that the D7 is holomorphically embedded into the Calabi-Yau and so holomorphic $\gamma$-matrices in the bulk are pulled-back to holomorphic $\gamma$-matrices. On the other hand, the deformation of the worldvolume that we found here is non-holomorphic when expressed in terms of the unperturbed complex structure. For example, for a Kuperstein in DKM, the deformed embedding can be written as $z^{4}=f(u)$ where $u$ is a real, as opposed to holomorphic,

\footnotetext{
${ }^{16}$ Due to the localized nature of the source of supersymmetry breaking and the RG filtering of UV effects, we anticipate that this discussion should be largely insensitive to the details of the UV completion of the warped geometry that is necessary to perform the reduction.
} 
function of the other $z^{A}$. This implies that the pullback of a holomorphic $\gamma$-matrix to the worldvolume will contain both holomorphic and anti-holomorphic parts, inducing a coupling to $(2,1)$ components of the flux. This is similar to the effect discussed in [19] where the squashing of the metric can lead to contributions to the soft terms from other Hodge-types. When the deformation of the D7 is not taken into account, a probe Kuperstein in DKM does not receive a contribution to the gaugino mass as a result of the dimensional reduction [19]. The interpretation provided by [19] was the $Z_{2 M} \mathrm{R}-$ symmetry of the KT geometry (a remnant of the U (1) R-symmetry of KW under which $z^{i} \rightarrow \mathrm{e}^{\mathrm{i} \alpha} z^{i}$ ) which forbids a non-vanishing gaugino mass when $M>1$. At least morally, the terms appearing in the D7-geometry are essentially pullbacks of R-symmetric terms and so cannot induce a gaugino mass. However, the D7-brane explicitly breaks the R-symmetry entirely and hence one should not expect this protection to be perfect. Although we again defer a more precise treatment to future work, the effect of the non-holomorphic pullback is particularly sensitive to the R-breaking embedding of the D7 and hence we expect that effect to give a non-vanishing contribution. Furthermore, the deformation of the worldvolume, as characterized by (5.32), has the same scaling with $\mathcal{S}$ and $\mu$ (using (5.33)) as the non-supersymmetric deformations of the geometry (5.6). Therefore we expect this contribution to be comparable to the naive parametric estimates for the gaugino mass appearing in [19]. Similar arguments can be made for other soft terms resulting from the dimensional reduction. Hence, the deformation of the worldvolume of the D7s generically introduces $\mathcal{O}(1)$ corrections to the soft Lagrangian of the low-energy effective theory.

In the context of AdS/CFT, the inclusion of D7-branes corresponds to the addition of a global flavor symmetry and quarks that transform as fundamentals under this flavor group and the strongly coupled gauge group. The spectrum of mesons and mesini in the dual theory can be determined by considering the open-string fluctuations of the D7. This analysis was performed in [64] for a D7 probe $A d S^{5} \times S^{5}$ and in [52] for a subset of the mesons for a Kuperstein D7 in Klebanov-Strassler. The analyses were extended to the non-supersymmetric cases in [22] and [19] respectively. However in these cases the deformation of the D7 was taken not into account. One would again expect that in each of these setups the deformation would alter the spectrum by $\mathcal{O}(1)$ factors and subsequently the visible-sector soft terms found in models of holographic gauge mediation models (though we anticipate that the effect would not change the parametric scaling of such terms).

As discussed in the introduction, D7-branes are expected to appear in string theory realization of bulk Randall-Sundrum scenarios [5] (see, for example, [12, 65]). Although gauge multiplets appear on a single stack of D7-branes, bifundamental chiral matter appears on the intersection of magnetized D7-branes. One possibility (see figure 8 ) is to model the visible sector gauge group as $\mathrm{U}\left(N_{f}\right)$ realized on D7s characterized by an embedding parameter $\mu$ that intersects with another stack of D7s with a different embedding parameter ${ }^{17}$. If the intersection carries a chiral index (i.e. if the integral of $\mathcal{F}_{2}$ over the intersection is non-vanishing), then chiral modes will be supported on the intersection. In the unwarped case, the wavefunctions for the chiral modes are Gaussians peaked at different locations along the intersection with the spacing of the modes set by the chiral index (see e.g. [11]). Although the warping is difficult to take into account [66], it is reasonable to assume that this property persists to the warped case, though perhaps with some modulation due to the warping. As in RS models and other string constructions, operators in the $4 \mathrm{~d}$ effective field theory description depend on the overlap of such wavefunctions. As these wavefunctions will necessarily depend on the shape of the worldvolumes that support them, the deformations that we considered here must be taken into account.

\footnotetext{
${ }^{17}$ Note that in order for the branes to intersect, different coordinates for the embedding must be used. For Kuperstein embeddings for example, we might use $z^{4} \sim \mu$ for one stack and $z^{3} \sim \nu$ for the other.
} 


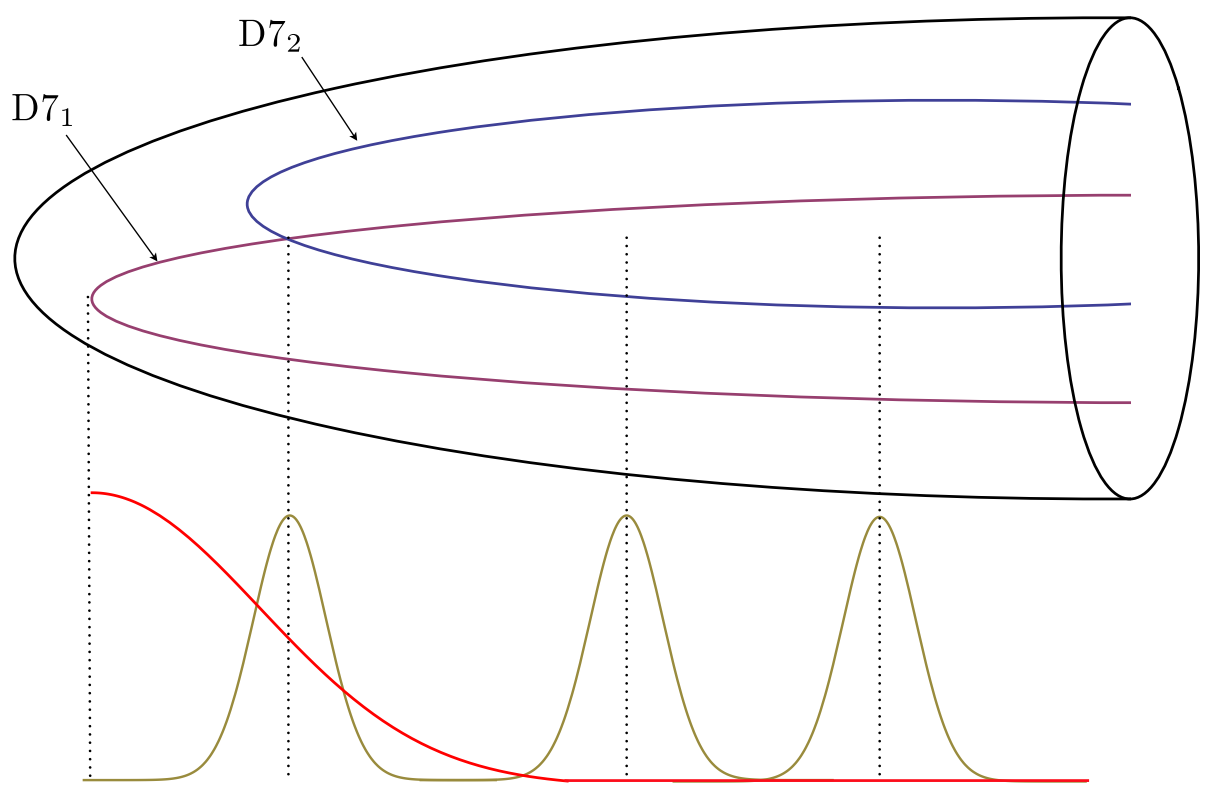

Figure 8. Cartoon of chiral modes supported by intersecting D7 branes in a warped geometry. D7 1 supports a scalar whose IR-localized profile is in red, while the magnetized intersection $\mathrm{D} 7_{1}$ and $\mathrm{D} 7_{2}$ supports chiral modes localized at various positions depending on the magnetic flux.

However, unlike some of the previous effects we discussed where the correction is not anticipated to generically lead to qualitatively different results, in this case there may be some qualitative differences. For example, in some supersymmetric string models only one generation of fermions receive masses from electroweak symmetry breaking effects (see, for example, [67, 68]). Although non-perturbative effects can resolve this rank-one problem, it would be important to understand to what extent the breaking of supersymmetry, and the corresponding deformation of D7 worldvolumes, alters the story.

Finally, we consider the effect of the inter-flavor forces induced by the breaking of supersymmetry that were discussed in section 3.3. In that analysis we found some evidence that the force between different flavor branes may be repulsive at small distances. This would imply that stacks of branes are, under certain circumstances, unstable in these geometries. In addition to making the construction of visible-sector non-Abelian gauge symmetries difficult, it could also interfere with the onset of gaugino condensation which is an essential ingredient in the moduli stabilization scheme of [41] and related constructions. We again emphasize that our analysis is in a very particular construction and we treated the D7 as a small perturbation which is inapplicable at small distances. Furthermore, in a complete treatment the D7 branes are stabilized partially by fluxes in the bulk and their effect must be considered as well. Finally, such an effect would be rather surprising from the dual field theory 
point of view. Hence, we refrain from making any definitive statement of the existence of such an instability, but merely point out that it is a possible concern worth exploring.

\section{Concluding remarks}

In this paper we have examined the behavior of D7-branes in non-supersymmetric geometries. In particular, we focused on GKP-like warped geometries where the backreaction of D7-branes can be controlled. In such cases, $\overline{\mathrm{D} 3}$-branes naturally sit at origin of the geometry and break supersymmetry in a relatively well-controlled (and possibly metastable) manner. We argued that in such a setup, D7s experience a small attractive force to the 3 -branes. Since we impose that the D7s asymptote to their unperturbed configurations, this results in a small bending of the flavor branes. In the $A d S^{5} \times X^{5}$ case, the bending falls off as $r_{\min }^{-8}$ where $r_{\min }$ is the distance of closest approach before the $\overline{\mathrm{D} 3} \mathrm{~s}$ are added. In the case of $\overline{\mathrm{D} 3} \mathrm{~s}$ in the KS geometry (which we modeled as the DKM perturbation of KT), the effect falls off as $r_{\text {min }}^{-4}$. In either case, the influence on the D7 physics (for example soft terms) from this bending is comparable to the effects produced when the deformation of the worldvolume is neglected.

Beyond a more quantitative analysis of the effects on model building, there are a number of ways in which our analysis can be improved. For example, as discussed in section 5, the DKM solution [18] only captures some of the behavior of the backreaction of $\overline{\mathrm{D} 3} \mathrm{~s}$ in the Klebanov-Strassler geometry. In addition to terms that scale as $r^{-8}$ that are present even in KT, there are terms that scale as $r^{-3}$ [33] but vanish as we take the deformation parameter of the deformed conifold is taken to zero. Since the size of this deformation is set by the amount of flux in the geometry, we cannot literally take this limit, and hence it may be worth while to understand how the effects we compute are modified when we include this deformation.

We have also examined how the effects of backreacting flavors will modify the above picture and found some hints that at long distances the flavor branes attract each other while at smaller distances they repel. To make progress in addressing the backreaction of the D7s, we considered the smeared approximation and furthermore considered only the backreaction of "massless" D7s, meaning those whose quarks in the dual theory are massless. Furthermore, due to the Landau pole in the dual theory, the geometry resulting from such backreaction becomes singular in the UV. It would be worth understanding how to get around both of these issues ${ }^{18}$. In particular, smearing the D7s is a rather dramatic approximation to make, especially in light of the fact the D7s will exert forces on each other. Although smearing may, to linear order, correctly capture the radial components of the inter-brane forces, in general there will be angular components that would probably be important. It would also be interesting to understand how more general 7-branes behave ${ }^{19}$ However, such an analysis would require an F-theoretical approach and little has been done in non-supersymmetric F-theory constructions. One of the consequences of a repulsion between D7s in this sort of setup is the difficulty in constructing non-Abelian gauge groups in the low-energy theory. Although our analysis is not sufficient to make a strong statement regarding the existence of this repulsion, it may be important to understand the interaction at small distances.

Finally, our analysis was entirely in the context of a supergravity description of the branes. An interesting and complimentary approach would be a worldsheet analysis by considering the exchange of closed strings between a D3-D3-pair and a "parallel" D7-brane. Such a calculation can confirm, for

\footnotetext{
${ }^{18} \mathrm{It}$ is worth noting that some progress has been made in understanding the non-smeared solutions (see, for example, $[69,70])$.

${ }^{19} \overline{\mathrm{D} 7}$ s may also be interesting to consider as in, for example, [71].
} 
example, the absence of a force between D3s and D7s. However, such a computation may be somewhat involved due to the non-BPS stack of 3-branes.

\section{Acknowledgments}

It is a pleasure to thank Ben Heidenreich, Cody Long, and Liam McAllister for helpful discussions. I also thank Gary Shiu and Yoske Sumitomo for past conversations on closely related topics which piqued my initial interest in this question. This work was supported by the NSF under grant PHY-0757868.

\section{A Type-IIB supergravity}

The bosonic degrees of freedom of type-IIB supergravity consist of the 10d Einstein-frame metric $\hat{g}_{M N}=g_{\mathrm{s}}^{1 / 2} \mathrm{e}^{-\phi / 2} \hat{g}_{M N}^{\text {string }}$, the axiodilaton $\tau=C_{0}+\mathrm{ie}^{-\phi}$ (normalized such that the IIB coupling is $\left.g_{\mathrm{IIB}}=(\operatorname{Im} \tau)^{-1}\right)$, the 2 -form potentials $B_{2}$ and $C_{2}$, and the 4 -form potential $C_{4}$. In terms of the gauge-invariant field strengths

$$
H_{3}=\mathrm{d} B_{2}, \quad F_{1}=\mathrm{d} C_{0}, \quad F_{3}=\mathrm{d} C_{2}-H_{3} C_{0}, \quad G_{3}=F_{3}-\mathrm{ie}^{-\phi} H_{3}, \quad F_{5}=\mathrm{d} C_{4}-H_{3} \wedge C_{2},
$$

the corresponding pseudo-action is

$$
\begin{aligned}
S_{\text {IIB }}=\frac{1}{2 \kappa_{10}^{2}} \int\{\hat{*} \hat{R} & +\frac{1}{2(\operatorname{Im} \tau)^{2}} \mathrm{~d} \tau \wedge \hat{*} \mathrm{~d} \bar{\tau}+\frac{g_{\mathrm{s}}}{2 \operatorname{Im} \tau} G_{3} \wedge \hat{*} \bar{G}_{3} \\
& \left.+\frac{g_{\mathrm{s}}^{2}}{4} F_{5} \wedge \hat{*} F_{5}+\frac{\mathrm{i} g_{\mathrm{s}}^{2}}{4 \operatorname{Im} \tau} C_{4} \wedge G_{3} \wedge \bar{G}_{3}\right\}
\end{aligned}
$$

in which $2 \kappa_{10}^{2}=(2 \pi)^{7} \alpha^{4} g_{\mathrm{s}}^{2}, \hat{R}$ is the Ricci scalar built from $\hat{g}_{M P}$ and we have defined the Hodge- $*$ in $D$ dimensions by

$$
\left(\hat{*} \Omega_{p}\right)_{M_{1} \cdots M_{D-p}}=\frac{1}{p !} \hat{\epsilon}_{M_{1} \cdots M_{D-p}}{ }^{N_{1} \cdots N_{p}} \Omega_{N_{1} \cdots N_{p}},
$$

where we make use of the 10d volume form

$$
\hat{\epsilon}_{0 \ldots 9}=+\sqrt{-\operatorname{det}(\hat{g})} .
$$

The resulting equations of motion must be supplemented by the self-duality constraint

$$
F_{5}=\hat{*} F_{5} .
$$

We will couple the supergravity modes to D3-branes, $\overline{\mathrm{D} 3}$-branes, and D7-branes ${ }^{20}$. The bosonic action for a $\mathrm{D} p$-brane is a sum of the Dirac-Born-Infeld and Chern-Simons actions

$$
\begin{aligned}
& S_{\mathrm{D} p^{ \pm}}=S_{\mathrm{D} p}^{\mathrm{DBI}}+S_{\mathrm{D} p^{ \pm}}^{\mathrm{CS}}, \\
& S_{\mathrm{D} p}^{\mathrm{DBI}}=-\tau_{\mathrm{D} p} \int \mathrm{d}^{p+1} \xi\left(g_{\mathrm{s}}^{-1} \mathrm{e}^{\phi}\right)^{(p-3) / 4} \sqrt{-\operatorname{det}\left(\mathrm{P}\left[\hat{g}+g_{\mathrm{s}}^{1 / 2} \mathrm{e}^{-\phi / 2} B\right]_{\alpha \beta}+2 \pi \alpha^{\prime} g_{\mathrm{s}}^{1 / 2} \mathrm{e}^{-\phi / 2} f_{\alpha \beta}\right)}, \\
& S_{\mathrm{D} p^{ \pm}}^{\mathrm{CS}}= \pm \tau_{\mathrm{D} p} \int \mathrm{P}\left[\mathcal{C} \wedge \mathrm{e}^{B_{2}}\right] \wedge \mathrm{e}^{2 \pi \alpha^{\prime} f_{2}},
\end{aligned}
$$

\footnotetext{
${ }^{20}$ We will not work with explicit compactifications so that we need not consider orientifold planes.
} 
in which $\tau_{\mathrm{D} p}^{-1}=(2 \pi)^{p} \alpha^{\prime(p+1) / 2} g_{\mathrm{s}}$ gives the Einstein-frame brane tension, $\mathrm{P}$ denotes the pullback to the worldvolume of the brane, and $f_{2}=\mathrm{d} a_{1}$ is the field strength of the $\mathrm{U}(1)$ gauge field supported by the brane. In the Chern-Simons action, the upper sign applies to D $p$-branes and the lower sign to $\overline{\mathrm{D} p}$-branes. $\mathcal{C}$ is the formal sum of Ramond-Ramond potentials and their magnetic duals defined by

$$
F_{7}=\mathrm{d} C_{6}-H_{3} \wedge C_{4}=-g_{\mathrm{s}}^{-1} \mathrm{e}^{\phi} \hat{*} F_{3}, \quad F_{9}=\mathrm{d} C_{8}-H_{3} \wedge C_{6}=g_{\mathrm{s}}^{-2} \mathrm{e}^{2 \phi} \hat{*} \mathrm{~d} F_{1},
$$

in which the factors of the string coupling appear because we are working in the 10d Einstein frame while these forms are more naturally defined in the string frame.

\section{B Backreacting 3-branes on Calabi-Yau cones}

In this appendix we consider the backreaction of $N$ D3-branes and $p \ll N$ D3- $\overline{\mathrm{D} 3}$ pairs at the tip of a Calabi-Yau cone. The ansatz and relevant field definitions are reviewed in section 2. For the internal metric we take the ansatz

$$
\mathrm{d} s_{6}^{2}=\mathrm{e}^{2 B}\left(\mathrm{~d} r^{2}+r^{2} \mathrm{~d} s_{X^{5}}^{2}\right),
$$

in which $\mathrm{d} s_{X^{5}}^{2}=\breve{g}_{\theta \phi} \mathrm{d} y^{\theta} \mathrm{d} y^{\phi}$ is the metric on a Sasaki-Einstein space normalized such that its Ricci tensor is $\breve{R}_{\theta \phi}=4 \breve{g}_{\theta \phi}$. One way to proceed in the flat space case would be to take a small $\frac{p}{N}$ limit of the exact supergravity solutions presented in [46]. However, our interest is in the near-brane limit in which the space asymptotes to $A d S^{5} \times X^{5}$ rather than flat space as assumed in [46]. The near-brane limit of the solution of [46] was performed and presented to leading order in $\frac{p}{N}$ in [18].

In this section we review and extend the result of [18]. We will exploit the observation of [9] that the supergravity equations of motion describing a perturbation from a background with $\Phi_{-}=0$, $G_{-}=0$, and constant dilaton take a block-triangular form. Moreover, this structure persists orderby-order in perturbation theory. Before taking the near-brane limit, the backreaction of $N$ D3s at the tip of the cone takes the form

$$
\Phi_{+}^{-1}=\frac{1}{2}+\frac{L^{4}}{2 r^{4}}, \quad \tau=\frac{\mathrm{i}}{g_{\mathrm{s}}}, \quad \mathrm{e}^{2 B}=1, \quad L^{4}=\frac{4 \pi^{4} g_{\mathrm{s}} N \alpha^{\prime 2}}{\mathcal{V}_{X^{5}}},
$$

in which $\mathcal{V}_{X^{5}}$ is the volume of the compact Sasaki-Einstein space and all other fields vanish. We then perturb this geometry by the addition of D3- $\overline{\mathrm{D} 3}$ pairs. In doing so, we can take $G_{ \pm}$and $\tau$ to remain trivial since the $\overline{\mathrm{D} 3}$ is not charged under any of the relevant fields and they are trivial before the addition of the $\overline{\mathrm{D} 3} \mathrm{~s}$. The equations of motion (2.7) take the form

$$
\begin{aligned}
& 0=\Phi_{-}^{\prime \prime}+\left(4 B^{\prime}+\frac{5}{r}\right) \Phi_{-}^{\prime}-\frac{2 \Theta_{+}}{1+\Phi_{-} \Theta_{+}} \Phi_{-}^{\prime 2}-\frac{\left(1+\Phi_{-} \Theta_{+}\right)^{2}}{2 \Theta_{+}^{2}}\left(4 \pi^{2} \alpha^{\prime}\right)^{2} p g_{\mathrm{s}} \frac{\delta^{6}(y)}{r^{5} \mathrm{e}^{4 B}}, \\
& 0=-5 B^{\prime \prime}-\frac{5}{r} B^{\prime}+\frac{2}{\left(1+\Phi_{-} \Theta_{+}\right)^{2}} \Theta_{+}^{\prime} \Phi_{-}^{\prime}, \\
& 0=B^{\prime \prime}+4 B^{\prime 2}+\frac{9}{r} B^{\prime} \\
& 0=\Theta_{+}^{\prime \prime}+\left(4 B^{\prime}+\frac{5}{r}\right) \Theta_{+}^{\prime}-\frac{2 \Phi_{-}}{1+\Phi_{-} \Theta_{+}} \Theta_{+}^{\prime 2}+\frac{\left(1+\Phi_{-} \Theta_{+}\right)^{2}}{2}\left(4 \pi^{2} \alpha^{\prime}\right)^{2}(N+p) g_{\mathrm{s}} \frac{\delta^{6}(y)}{r^{5} \mathrm{e}^{4 B}}
\end{aligned}
$$

in which we have defined $\Theta_{+}=\Phi_{+}^{-1}$ and have taken all of the fields to depend only on $r$. The Ricci tensor in the perturbed geometry is

$$
R_{r r}=-5 B^{\prime \prime}-\frac{5}{r} B^{\prime}, \quad R_{\theta \phi}=-r^{2}\left(B^{\prime \prime}+4 B^{\prime 2}+\frac{9}{r} B^{\prime}\right) \breve{g}_{\theta \phi} .
$$


Since we are treating $\frac{p}{N}$ as a small parameter, it useful to expand the fields as a power series in $\frac{p}{N}$. To that end, we write

$$
\Phi_{-}=\sum_{n=0}^{\infty}\left(\frac{p}{N}\right)^{n} \Phi_{-}^{(n)},
$$

and similarly for other fields. The equations of motion are then straightforwardly solved order-by-order in $\frac{p}{N}$. At any given order, we first first solve (B.3a), followed by (B.3b) and (B.3c) and finally (B.3d). The result is

$$
\begin{aligned}
\Phi_{-} & =-\frac{p}{N} \frac{2 L^{4}}{r^{4}}+\left(\frac{p}{N}\right)^{2}\left(\frac{2 L^{8}}{r^{8}}+\frac{2 L^{12}}{5 r^{12}}\right), \\
B & =-\frac{p}{N} \frac{L^{8}}{10 r^{8}}+\left(\frac{p}{N}\right)^{2}\left(-\frac{L^{8}}{10 r^{8}}-\frac{L^{16}}{50 r^{16}}\right), \\
\Theta_{+} & =\frac{1}{2}+\frac{L^{4}}{2 r^{4}}+\frac{p}{N}\left(\frac{L^{4}}{2 r^{4}}-\frac{L^{12}}{10 r^{12}}\right)+\left(\frac{p}{N}\right)^{2}\left(-\frac{L^{12}}{5 r^{12}}+\frac{2 L^{20}}{125 r^{20}}\right) .
\end{aligned}
$$

The integration constants have been fixed by integrating (B.3a) and (B.3d) and by requiring that the space asymptotes to the $R^{9,1}$. The warp factor for this solution is

$$
\mathrm{e}^{-4 A}=1+\frac{L^{4}}{r^{4}}+\frac{p}{N}\left(\frac{2 L^{4}}{r^{4}}+\frac{2 L^{8}}{r^{8}}+\frac{4 L^{12}}{5 r^{12}}\right)+\cdots
$$

To obtain the near-horizon limit, we follow [18] and perform the rescaling

$$
x^{\mu}=\tilde{x}^{\mu} Z^{-1 / 4}, \quad r=\tilde{r} Z^{-1 / 4}, \quad L^{4}=\tilde{L}^{4} Z^{-2}, \quad N=\tilde{N} Z^{-2}, \quad p=\tilde{p},
$$

and then consider the limit in which $Z \rightarrow 0$ while holding fixed $\tilde{L}$ and other quantities with tildes. Then, $\mathrm{e}^{2 A} \mathrm{~d} x_{4}^{2}=Z^{-1 / 2} \mathrm{e}^{2 A} \mathrm{~d} x_{4}^{2}$ and so the warp factor in the near-brane limit is $\mathrm{e}^{2 \tilde{A}}=Z^{-1 / 2} \mathrm{e}^{2 A}$. Taking $Z \rightarrow 0$ and dropping the tildes for notational simplicity, we find

$$
\begin{aligned}
\mathrm{e}^{-4 A} & =\frac{L^{4}}{r^{4}}+\frac{4 p}{5 N} \frac{L^{12}}{r^{12}}+\frac{54 p^{2}}{125 N^{2}} \frac{L^{20}}{r^{20}}, \\
\omega & =\frac{r^{4}}{L^{4}}+\frac{6 p}{5 N} \frac{L^{4}}{r^{4}}-\frac{24 p^{2}}{125 N^{2}} \frac{L^{12}}{r^{12}} \\
\mathrm{e}^{2 B} & =1-\frac{p}{5 N} \frac{L^{8}}{r^{8}}-\frac{p^{2}}{50 N^{2}} \frac{L^{16}}{r^{16}} .
\end{aligned}
$$

\section{Backreaction of smeared D7s}

\section{C.1 D7s and 3-branes in flat space}

Here we construct the supergravity solution corresponding to the backreaction of D7s smeared over the near-brane background created by D3s and $\overline{\mathrm{D} 3}$ s in flat space. The strategy will be to first review the backreaction of D7s in $A d S^{5} \times S^{5}$ and then perturb the resulting geometry by $\overline{\mathrm{D} 3}$-branes. Our starting point is the warped ansatz (2.4) where $g_{m n}$ is a metric on $R^{6} \simeq C^{3}$ which we express as (3.4). In terms of the radial coordinate defined by (3.3), it useful to define a new radial coordinate $\rho$ by

$$
r=\alpha^{1 / 2} \mathrm{e}^{\rho} .
$$

Then the metric (3.4) on $C^{3}$ takes the form

$$
\mathrm{d} s_{6}^{2}=\alpha^{\prime} \mathrm{e}^{2 \rho}\left\{\mathrm{d} \rho^{2}+(\mathrm{d} \psi+\mathcal{A})^{2}+\mathrm{d} s_{C P^{2}}^{2}\right\} .
$$


We then consider a D7-brane embedded into the geometry according to (3.9)

$$
z^{3}=\alpha^{1 / 2} \mathrm{e}^{\rho_{\nu}},
$$

in which $\rho_{\nu}$ is a constant. The backreaction of such codimension two objects is quite difficult to compute and so we consider a smeared approximation in which we take large number of D7s distributed in such a way that they can be treated as uniformly spread along the internal space. Here we follow the smearing suggested by [44] and implemented for this configuration in $A d S^{5} \times S^{5}$ in [48] which we quickly review. An $\mathrm{SU}(3) \times \mathrm{U}(1)$ rotation of the embedding (C.3) gives

$$
a_{i} z^{i}=\alpha^{1 / 2} \mathrm{e}^{\rho_{\nu}+\mathrm{i} \alpha}
$$

where $\alpha \in[0,2 \pi)$ while the $a_{i}$ are complex numbers satisfying

$$
a_{i} \bar{a}_{i}=1 .
$$

The $a_{i}$ thus parametrize a unit $S^{5}$, which we denote $\tilde{S}^{5}$, and so we can write

$$
\begin{aligned}
& a_{1}=\cos \frac{\tilde{\gamma}}{2} \cos \frac{\tilde{\theta}}{2} \mathrm{e}^{\mathrm{i}(\tilde{\psi}+\tilde{\eta} / 2+\tilde{\varphi} / 2)} \\
& a_{2}=\cos \frac{\tilde{\gamma}}{2} \sin \frac{\tilde{\theta}}{2} \mathrm{e}^{\mathrm{i}(\tilde{\psi}+\tilde{\eta} / 2-\tilde{\varphi} / 2)}, \\
& a_{3}=\sin \frac{\tilde{\gamma}}{2} \mathrm{e}^{\mathrm{i} \tilde{\psi}}
\end{aligned}
$$

in which, as before, $\tilde{\gamma}, \tilde{\theta} \in[0, \pi], \tilde{\varphi}, \tilde{\psi} \in[0,2 \pi)$, and $\tilde{\eta} \in[0,4 \pi)$. The distribution of D7s is specified by a density function on $\tilde{S}^{5} \times[0,2 \pi)$. The density function $\rho_{\mathrm{D} 7}$ is normalized such that

$$
N_{f}=\int \operatorname{dvol}_{\tilde{S}^{5}} \mathrm{~d} \alpha \rho_{\mathrm{D} 7}
$$

in which $N_{f}$ is the total number of D7s. A uniform distribution corresponds to a constant $\rho_{\mathrm{D} 7}$

$$
\rho_{\mathrm{D} 7}=\frac{N_{f}}{2 \pi^{4}} .
$$

With this configuration of magnetic charges for $C_{0}, F_{1}$ must take the form ${ }^{21}$

$$
F_{1}=-Q(\rho)(\mathrm{d} \psi+\mathcal{A}) .
$$

Writing the CS action for the D7s as

$$
\tau_{\mathrm{D} 7} g_{\mathrm{s}} \sum_{\mathrm{D} 7} \int_{\mathcal{W}^{i}} C_{8}=\tau_{\mathrm{D} 7} g_{\mathrm{s}} \sum_{\mathrm{D} 7} \int \Omega_{i} \wedge C_{8}=: \tau_{\mathrm{D} 7} g_{\mathrm{s}} \int \Omega \wedge C_{8}
$$

we have

$$
\mathrm{d} F_{1}=-\Omega .
$$

We can write the embedding (C.4) as $f_{1}=f_{2}=0$ in which

$$
\begin{aligned}
& f_{1}=2(\psi+\tilde{\psi})+\eta+\tilde{\eta}+\varphi+\tilde{\varphi}+2 \arg \Gamma-2 \alpha+4 \pi n, \\
& f_{2}=|\Gamma|^{2}-\mathrm{e}^{2\left(\rho_{\nu}-\rho\right)},
\end{aligned}
$$

\footnotetext{
${ }^{21}$ Note that the relative sign with respect to the solutions reviewed in [45] is conventional.
} 
with $n \in Z$ and

$$
\Gamma=\cos \frac{\gamma}{2} \cos \frac{\tilde{\gamma}}{2} \cos \frac{\theta}{2} \cos \frac{\tilde{\theta}}{2}+\cos \frac{\gamma}{2} \cos \frac{\tilde{\gamma}}{2} \sin \frac{\theta}{2} \sin \frac{\tilde{\theta}}{2} \mathrm{e}^{-\mathrm{i}(\varphi+\tilde{\varphi})}+\sin \frac{\gamma}{2} \sin \frac{\tilde{\gamma}}{2} \mathrm{e}^{-\frac{\mathrm{i}}{2}(\eta+\tilde{\eta}+\varphi+\tilde{\varphi})} .
$$

Then

$$
\Omega=\int \operatorname{dvol}_{\tilde{S}^{5}} \mathrm{~d} \alpha \rho^{\mathrm{D} 7} \delta\left(f_{1}\right) \delta\left(f_{2}\right) \mathrm{d} f_{1} \wedge \mathrm{d} f_{2},
$$

where we are again integrating over the space $\tilde{S}^{5} \times[0,2 \pi)$ and the $\mathrm{d} f_{i}$, which are 1 -forms in the physical space $C^{3}$, are not integrated over. Due to the isometries we can evaluate the integral at particular point in $S^{5}$ (for example, $\gamma=\pi$ is a particularly simple choice) and we find a distribution consistent with the ansatz for $F_{1}$ with [48]

$$
Q=\left\{\begin{array}{ll}
0 & \rho<\rho_{\nu} \\
\frac{N_{f}}{2 \pi}\left(1-\mathrm{e}^{2\left(\rho_{\nu}-\rho\right)}\right)^{2} & \rho \geq \rho_{\nu}
\end{array} .\right.
$$

We then take the limit in which $\rho_{\nu} \rightarrow \infty$, corresponding to the massless limit of the quarks in the dual theory.

The D7s backreact on the metric and with this smearing procedure the most general metric consistent with the isometries that we can write is

$$
\mathrm{d} s_{6}^{2}=\alpha^{\prime}\left\{\mathrm{e}^{2 f}\left[\mathrm{~d} \rho^{2}+(\mathrm{d} \psi+\mathcal{A})^{2}\right]+\mathrm{e}^{2 g} \mathrm{~d} s_{C P^{2}}^{2}\right\} .
$$

The metric functions and other fields can be solved by an analysis of the Killing spinor equations. The result is [44]

$$
\begin{aligned}
\mathrm{e}^{-\phi} & =\frac{1}{g_{\mathrm{s}}}\left\{1-\frac{g_{\mathrm{s}} N_{f}}{2 \pi}\left(\rho-\rho_{\mathrm{s}}\right)\right\}, \\
\mathrm{e}^{2 g} & =c_{1} \mathrm{e}^{2 \rho}\left\{1-\frac{g_{\mathrm{s}} N_{f}}{2 \pi}\left[\left(\rho-\rho_{\mathrm{s}}\right)-\frac{1}{6}+c_{2} \mathrm{e}^{-6 \rho}\right]\right\}^{1 / 3}, \\
\mathrm{e}^{2 f} & =c_{1} \mathrm{e}^{2 \rho} \frac{1-\frac{g_{\mathrm{s}} N_{f}}{2 \pi}\left(\rho-\rho_{\mathrm{s}}\right)}{\left\{1-\frac{g_{\mathrm{s}} N_{f}}{2 \pi}\left[\left(\rho-\rho_{\mathrm{s}}\right)-\frac{1}{6}+c_{2} \mathrm{e}^{-6 \rho}\right]\right\}^{2 / 3}}, \\
\mathrm{e}^{-4 A} & =-16 \pi g_{\mathrm{s}} N \int \mathrm{d} \rho \mathrm{e}^{-4 g(\rho)}+c_{3} .
\end{aligned}
$$

The solution becomes singular as we take $\rho \rightarrow-\infty$, but the divergence can be made more mild by setting $c_{2}=0$; this can be thought of as the result of imposing IR singularity in the massive case and then taking the limit in which the mass vanishes [72]. The solution exhibits a Landau pole at $\rho=\rho_{\mathrm{LP}}=\rho_{\mathrm{s}}+\frac{2 \pi}{g_{\mathrm{s}} N_{f}}$ where $\mathrm{e}^{-\phi} \rightarrow 0$ and hence the coupling in the dual gauge theory diverges. We choose $c_{3}=0$ so that, as in [72], $\mathrm{e}^{-4 A}=0$ at the Landau pole. Finally, $c_{1}$ rescales the warp factor and so can be set to 1 by rescaling the Minkowski directions ${ }^{22}$. With these choices, the solution describing

\footnotetext{
${ }^{22}$ These conditions are a little arbitrary. In principle, they would be fixed after the geometry is completed in the UV to resolve the Landau pole. In any case, we will mostly be interested in physics at much lower scales where the corrections from a more precise set of boundary conditions will be exponentially small.
} 
the backreaction of smeared D7 branes in $A d S^{5} \times S^{5}$ is

$$
\begin{aligned}
\mathrm{e}^{-\phi} & =\frac{1}{g_{\mathrm{s}}}\left\{1-\frac{g_{\mathrm{s}} N_{f}}{2 \pi}\left(\rho-\rho_{\mathrm{s}}\right)\right\}, \\
\mathrm{e}^{2 g} & =\mathrm{e}^{2 \rho}\left\{1-\frac{g_{\mathrm{s}} N_{f}}{2 \pi}\left[\left(\rho-\rho_{\mathrm{s}}\right)-\frac{1}{6}\right]\right\}^{1 / 3}, \\
\mathrm{e}^{2 f} & =\mathrm{e}^{2 \rho} \frac{1-\frac{g_{\mathrm{s}} N_{f}}{2 \pi}\left(\rho-\rho_{\mathrm{s}}\right)}{\left\{1-\frac{g_{\mathrm{s}} N_{f}}{2 \pi}\left[\left(\rho-\rho_{\mathrm{s}}\right)-\frac{1}{6}\right]\right\}^{2 / 3}}, \\
\mathrm{e}^{-4 A} & =-16 \pi g_{\mathrm{s}} N \int_{\rho_{\mathrm{LP}}}^{\rho} \mathrm{d} x \mathrm{e}^{-4 g(x)} .
\end{aligned}
$$

Because the above configuration is supersymmetric, we are guaranteed that the supergravity and D7-brane equations of motion are satisfied. However, when supersymmetry is broken the Killing spinor analysis that lead to the above solution does not apply and the equations of motion must be explicitly considered. We begin by recasting the equations of motion (2.7). The charge distribution $\Omega$ is generically not decomposable but we can express it as a sum of decomposable pieces. In particular, since $Q$ is a constant we have, for our smeared approximation,

$$
\Omega=2 Q \mathcal{J}
$$

where $\mathcal{J}$ is the Kähler form on $C P^{2}$. Still following $[44,48]$ we define a local frame $\left\{e^{\underline{\rho}}, e^{\underline{\underline{0}}}, e^{\underline{\underline{a}}}\right\}$ via the vielbein

$$
e_{\rho}^{\underline{\rho}}=\alpha^{1 / 2} \mathrm{e}^{f}, \quad e_{\psi}^{\underline{0}}=\alpha^{1 / 2} \mathrm{e}^{f}, \quad e_{a}^{\underline{0}}=\alpha^{1 / 2} \mathrm{e}^{f} \mathcal{A}_{a}, \quad e_{a}^{\underline{b}}=\alpha^{1 / 2} \mathrm{e}^{g} \tilde{e}_{a}^{\underline{b}},
$$

in which we have written the coordinates on $C P^{2}$ as $\mathrm{d} x^{a}$ and have chosen a frame $\tilde{e}^{\underline{a}}$ such that

$$
\mathcal{J}=\tilde{e}^{1} \wedge \tilde{e}^{2}+\tilde{e}^{3} \wedge \tilde{e}^{4}
$$

Hence we can write

$$
\Omega=\Omega_{1}+\Omega_{2}, \quad \Omega_{1}=2 Q \tilde{e}^{1} \wedge \tilde{e}^{2}, \quad \Omega_{2}=2 Q \tilde{e}^{3} \wedge \tilde{e}^{4}
$$

In this local frame, we find that the components of the Ricci tensor built from $g_{m n}$ are

$$
\begin{aligned}
& R_{\underline{\rho \rho}}=\frac{1}{\alpha^{\prime}} \mathrm{e}^{-2 f}\left\{-\partial_{\rho}^{2} f-4 \partial_{\rho}^{2} g-4\left(\partial_{\rho} g\right)^{2}+4 \partial_{\rho} f \partial_{\rho} g\right\}, \\
& R_{\underline{00}}=\frac{1}{\alpha^{\prime}} \mathrm{e}^{-2 f}\left\{-\partial_{\rho}^{2} f-4 \partial_{\rho} f \partial_{\rho} g+4 \mathrm{e}^{4(f-g)}\right\}, \\
& R_{\underline{a b}}=\frac{1}{\alpha^{\prime}} \mathrm{e}^{-2 f}\left\{-\partial_{\rho}^{2} g-4\left(\partial_{\rho} g\right)^{2}-2 \mathrm{e}^{4(f-g)}+6 \mathrm{e}^{2(f-g)}\right\} \delta_{\underline{a b}} .
\end{aligned}
$$

Then, using the above form for $\Omega_{i}$, the equations of motion $(2.7)$ take the form (where again $\Theta_{+}=\Phi_{+}^{-1}$ )

$$
\begin{aligned}
& 0=\partial_{\rho}^{2} \Phi_{-}+4 \partial_{\rho} g \partial_{\rho} \Phi_{-}-\frac{2 \Theta_{+}}{1+\Phi_{-} \Theta_{+}} \partial_{\rho} \Phi_{-} \partial_{\rho} \Phi_{-}, \\
& 0=\mathrm{e}^{-2 f}\left\{\partial_{\rho}^{2} \mathrm{e}^{-\phi}+4 \partial_{\rho} g \partial_{\rho} \mathrm{e}^{-\phi}\right\}+\mathrm{e}^{\phi-2 f}\left\{Q^{2}-\left(\partial_{\rho} \mathrm{e}^{-\phi}\right)^{2}\right\}+4 Q \mathrm{e}^{-2 g}, \\
& 0=-\partial_{\rho}^{2} f-4 \partial_{\rho}^{2} g-4\left(\partial_{\rho} g\right)^{2}+4 \partial_{\rho} f \partial_{\rho} g+\frac{2}{\left(1+\Theta_{+} \Phi_{-}\right)^{2}} \partial_{\rho} \Theta_{+} \partial_{\rho} \Phi_{-}-\frac{1}{2} \mathrm{e}^{2 \phi}\left(\partial_{\rho} \mathrm{e}^{-\phi}\right)^{2},
\end{aligned}
$$




$$
\begin{aligned}
& 0=-\partial_{\rho}^{2} f-4 \partial_{\rho} f \partial_{\rho} g+4 \mathrm{e}^{4(f-g)}-\frac{1}{2} \mathrm{e}^{2 \phi} Q^{2}, \\
& 0=-\partial_{\rho}^{2} g-4\left(\partial_{\rho} g\right)^{2}-2 \mathrm{e}^{4(f-g)}+6 \mathrm{e}^{2(f-g)}-Q \mathrm{e}^{\phi} \mathrm{e}^{2(f-g)}, \\
& 0=\partial_{\rho}^{2} \Theta_{+}+4 \partial_{\rho} g \partial_{\rho} \Theta_{+}-\frac{2 \Phi_{-}}{1+\Phi_{-} \Theta_{+}} \partial_{\rho} \Theta_{+} \partial_{\rho} \Theta_{+},
\end{aligned}
$$

where we have suppressed the appearance of explicit D3-branes. It is easy to confirm that (C.18) satisfies these equations.

We now perturb the geometry by the addition of $p$ D3- $\overline{\mathrm{D} 3}$ pairs. The equations are difficult to solve, even treating $\frac{p}{N}$ as a perturbation. To make progress, we will consider the case in which both the backreaction of D7s and the backreaction of the $\overline{\mathrm{D} 3} \mathrm{~s}$ can be treated as comparable perturbations.

First, largely following [48], we will argue that a D7 satisfying $z^{3}=0$ will still be a solution to the D7 equations of motion. To do so, we consider a brane filling the $\rho, \theta, \varphi$, and $\eta$ directions (where we use (3.3) and (C.1)) and take $\gamma$ and $\psi$ to be functions of $\rho$. The $z^{3}=0$ embedding is $\gamma=\psi=0$. From our analysis in section 3.1 we expect the Lagrangian describing the D7 fluctuations to be a function of $\left(\partial_{\rho} \psi\right)^{2},\left(\partial_{\rho} \eta\right)^{2}$, the worldvolume angles, and, in general, $\psi$ and $\gamma$ (as well as the worldvolume gauge fields, but those can again be consistently set equal to zero). To show that $\psi=\eta=0$ is a solution to the equations of motion, it then suffices to show that the $\frac{\delta \mathcal{L}}{\delta \psi}=\frac{\delta \mathcal{L}}{\delta \gamma}=0$ at these points. The D7 action again has two contributions, the DBI and CS piece. Setting $\psi$ and $\gamma$ to be constant, the induced metric on the D7 is

$$
\begin{aligned}
\mathrm{d} s_{4}^{2}=\alpha^{\prime}\left\{\mathrm{e}^{2 f}\left[\mathrm{~d} \rho^{2}+\frac{1}{4} \cos ^{4} \frac{\gamma}{2}(\mathrm{~d} \eta+\cos \theta \mathrm{d} \varphi)^{2}\right]\right. \\
\left.\quad+\mathrm{e}^{2 g}\left[\frac{1}{4} \cos ^{2} \frac{\gamma}{2}\left(\mathrm{~d} \theta^{2}+\sin ^{2} \theta \mathrm{d} \varphi^{2}\right)+\frac{1}{4} \cos ^{2} \frac{\gamma}{2} \sin ^{2} \frac{\gamma}{2}(\mathrm{~d} \eta+\cos \theta \mathrm{d} \varphi)^{2}\right]\right\} .
\end{aligned}
$$

All of the components are independent of $\psi$ and have vanishing derivatives with respect to $\gamma$ at $\gamma=0$. Furthermore, from the isometries the dilaton will depend only on $\rho$. Hence we have that $\frac{\delta \mathcal{L}^{\mathrm{DBI}}}{\delta \psi}=\frac{\delta \mathcal{L}^{\mathrm{DBI}}}{\delta \gamma}=0$ at $\psi=\gamma=0$.

Next we consider the CS contribution to the D7 action. If $z^{3}=0$ is a solution even after the D7s and $\overline{\mathrm{D} 3} \mathrm{~s}$ backreact, then the smearing of such branes will produce an $F_{1}$ given by

$$
F_{1}=-\frac{N_{f}}{2 \pi}(\mathrm{d} \psi+\mathcal{A})
$$

The field strength of the magnetic dual is

$$
F_{9} \sim \frac{N_{f}}{2 \pi} \mathrm{e}^{2 \phi+4 g} \operatorname{dvol}_{R^{3,1}} \wedge \mathrm{d} \rho \wedge \mathcal{J} \wedge \mathcal{J} .
$$

Since $\mathcal{J}=\frac{1}{2} \mathrm{~d} \mathcal{A}$ we can write

$$
C_{8} \sim \frac{N_{f}}{2 \pi} \mathrm{e}^{2 \phi+4 g} \operatorname{dvol}_{R^{3,1}} \wedge \mathrm{d} \rho \wedge \mathcal{J} \wedge \mathcal{A} .
$$

The CS action is proportional $\int \mathrm{P}\left[C_{8}\right]$ and so the corresponding Lagrangian behaves as

$$
\mathcal{L}^{\mathrm{CS}} \sim Q \mathrm{e}^{2 \phi+4 g} \sin \theta \cos ^{4} \frac{\gamma}{2},
$$


and hence $\frac{\delta \mathcal{L}^{\mathrm{CS}}}{\delta \psi}=\frac{\delta \mathcal{L}^{\mathrm{CS}}}{\delta \gamma}=0$ at $\gamma=\psi=0$. From this, we conclude that $\gamma=\psi=0$ is a solution to the D7 equations of motion even once the backreaction of such branes and $\overline{\mathrm{D} 3} \mathrm{~s}$ is taken into account. From the isometries of the problem, we immediately have that all embeddings of the type $a_{i} z^{i}=0$ solve the D7 equations of motion.

Treating the $\overline{\mathrm{D} 3} \mathrm{~s}$ and D7s as comparable perturbations, the equations of motion (B.3) can be simply solved by the method of undetermined coefficients. The result is

$$
\begin{aligned}
& \Phi_{-}=-8 \pi g_{\mathrm{s}} N \frac{p}{N} \mathrm{e}^{-4 \rho}-\frac{16}{3} \pi g_{\mathrm{s}} N \frac{g_{\mathrm{s}} N_{f}}{2 \pi} \frac{p}{N}\left(\rho-\rho_{\mathrm{s}}+\frac{1}{12}\right) \mathrm{e}^{-4 \rho} \\
&+\frac{128}{5}\left(\pi g_{\mathrm{s}} N\right)^{3} \frac{p^{2}}{N^{2}} \mathrm{e}^{-12 \rho}+\Phi_{-}^{\mathrm{LP}} \\
& \mathrm{e}^{-\phi}= \frac{1}{g_{\mathrm{s}}}\left\{1-\frac{g_{\mathrm{s}} N_{f}}{2 \pi}\left(\rho-\rho_{\mathrm{s}}\right)+\frac{8}{5}\left(\pi g_{\mathrm{s}} N\right)^{2} \frac{g_{\mathrm{s}} N_{f}}{2 \pi} \frac{p}{N} \mathrm{e}^{-8 \rho}\right\} \\
& \mathrm{e}^{2 g}= \mathrm{e}^{2 \rho}\left\{1-\frac{1}{3} \frac{g_{\mathrm{s}} N_{f}}{2 \pi}\left(\rho-\rho_{\mathrm{s}}-\frac{1}{6}\right)-\frac{16}{5}\left(\pi g_{\mathrm{s}} N\right)^{2} \frac{p}{N} \mathrm{e}^{-8 \rho}\right. \\
& \quad-\frac{1}{9}\left(\frac{g_{\mathrm{s}} N_{f}}{2 \pi}\right)^{2}\left[\left(\rho-\rho_{\mathrm{s}}\right)^{2}-\frac{1}{3}\left(\rho-\rho_{\mathrm{s}}\right)+\frac{1}{36}\right] \\
&\left.\quad-\frac{16}{5}\left(\pi g_{\mathrm{s}} N\right)^{2} \frac{g_{\mathrm{s}} N_{f}}{2 \pi} \frac{p}{N}\left(\rho-\rho_{\mathrm{s}}+\frac{1}{6}\right) \mathrm{e}^{-8 \rho}-\frac{128}{25}\left(\pi g_{\mathrm{s}} N\right)^{4}\left(\frac{p}{N}\right)^{2} \mathrm{e}^{-16 \rho}\right\} \\
& \mathrm{e}^{2 f}= \mathrm{e}^{2 \rho}\left\{1-\frac{1}{3} \frac{g_{\mathrm{s}} N_{f}}{2 \pi}\left(\rho-\rho_{\mathrm{s}}+\frac{1}{3}\right)-\frac{16}{5}\left(\pi g_{\mathrm{s}} N\right)^{2} \frac{p}{N} \mathrm{e}^{-8 \rho}\right. \\
& \quad-\frac{1}{9}\left(\frac{g_{\mathrm{s}} N_{f}}{2 \pi}\right)^{2}\left[\left(\rho-\rho_{\mathrm{s}}\right)^{2}+\frac{2}{3}\left(\rho-\rho_{\mathrm{s}}\right)-\frac{5}{36}\right] \\
&\left.\quad-\frac{16}{5}\left(\pi g_{\mathrm{s}} N\right)^{2} \frac{g_{\mathrm{s}} N_{f}}{2 \pi} \frac{p}{N}\left(\rho-\rho_{\mathrm{s}}\right) \mathrm{e}^{-8 \rho}-\frac{128}{25}\left(\pi g_{\mathrm{s}} N\right)^{4}\left(\frac{p}{N}\right)^{2} \mathrm{e}^{-16 \rho}\right\}, \\
& \Phi_{+}^{-1}=2 \pi g_{\mathrm{s}} N \mathrm{e}^{-4 \rho}+\frac{4 \pi}{3} g_{\mathrm{s}} N \frac{g_{\mathrm{s}} N_{f}}{2 \pi}\left(\rho-\rho_{\mathrm{s}}+\frac{1}{12}\right) \mathrm{e}^{-4 \rho}-\frac{32}{5}\left(\pi g_{\mathrm{s}} N\right)^{3} \frac{p}{N} \mathrm{e}^{-12 \rho} \\
&+\frac{10}{9} \pi g_{\mathrm{s}} N\left(\frac{g_{\mathrm{s}} N_{f}}{2 \pi}\right)^{2}\left[\left(\rho-\rho_{\mathrm{s}}\right)^{2}+\frac{1}{6}\left(\rho-\rho_{\mathrm{s}}\right)+\frac{5}{72}\right] \mathrm{e}^{-4 \rho} \\
&-\frac{64}{5}\left(\pi g_{\mathrm{s}} N\right)^{3} \frac{3 g_{\mathrm{s}} N_{f}}{2 \pi} \frac{p}{N}\left[\rho-\rho_{\mathrm{s}}+\frac{1}{12}\right] \mathrm{e}^{-8 \rho}+\frac{2048}{125}\left(\pi g_{\mathrm{s}} N\right)^{5}\left(\frac{p}{N}\right)^{2} \mathrm{e}^{-20 \rho}+\Theta_{+}^{\mathrm{LP}} .
\end{aligned}
$$

Integration constants were chosen so that when either $p=0$ or $N_{f}=0$ the solution matches what was previously found. This leaves unfixed a constant in the $\frac{p}{N} \frac{g_{s} N_{f}}{2 \pi}$ term which we chose by requiring $\Phi_{-}=-4 \frac{p}{N} \Phi_{+}^{-1}$ at $\mathcal{O}\left(\frac{p}{N}\right)$ (since the D7 effects enter the equations for $\Phi_{-}$and $\Phi_{+}^{-1}$ in the same way). The constants $\Phi_{-}^{\mathrm{LP}}$ and $\Theta_{+}^{\mathrm{LP}}$ are introduced to impose that $\mathrm{e}^{-4 A}$ and $\omega^{-1}$ vanish at the Landau pole. However, since the Landau poles occur where the perturbation treatment of the D7s breaks down, the constants cannot be reliably found. However, they should scale as $\mathrm{e}^{-4 \rho_{\mathrm{LP}}}$ and so will provide a negligible correction.

\section{C.2 Kuperstein branes on the conifold}

The backreaction of massive Kuperstein D7s in $A d S^{5} \times T^{1,1}$ was first considered in [73], though the massless case which to which we will specialize falls under the general analysis of [44].

For the conifold, we again define a radial coordinate $\rho$ by (C.1). The conifold metric is then

$$
\mathrm{d} s_{6}^{2}=\alpha^{\prime} \mathrm{e}^{2 \rho}\left\{\mathrm{d} \rho^{2}+\left(\frac{1}{3} \mathrm{~d} \psi+\mathcal{A}\right)^{2}+\mathrm{d} s_{S^{2} \times S^{2}}^{2}\right\} .
$$


Our fiducial Kuperstein embedding is then (4.12)

$$
z^{4}=\left(\frac{2}{27}\right)^{1 / 4} \alpha^{\prime 3 / 4} \mathrm{e}^{3 \rho_{\nu} / 2}
$$

A SO $(4) \times \mathrm{U}(1)$ rotation of this embedding is

$$
a_{A} z^{A}=\left(\frac{2}{27}\right)^{1 / 4} \alpha^{\prime 3 / 4} \mathrm{e}^{3 \rho_{\nu} / 2+\mathrm{i} \alpha},
$$

in which again $\alpha \in[0,2 \pi)$. The $a_{A}$ are real numbers satisfying

$$
a_{A} a_{A}=1,
$$

and so define an $S^{3}$ which we denote $\tilde{S}^{3}$. We can write

$$
\begin{aligned}
a_{1} & =\cos \frac{\tilde{\theta}}{2} \cos \frac{\tilde{\gamma}+\tilde{\varphi}}{2}, & a_{2} & =\cos \frac{\tilde{\theta}}{2} \sin \frac{\tilde{\gamma}+\tilde{\varphi}}{2}, \\
a_{3} & =\sin \frac{\tilde{\theta}}{2} \cos \frac{\tilde{\gamma}-\tilde{\varphi}}{2}, & a_{4} & =\sin \frac{\tilde{\theta}}{2} \sin \frac{\tilde{\gamma}-\tilde{\varphi}}{2},
\end{aligned}
$$

in which $\tilde{\theta} \in[0, \pi], \tilde{\varphi} \in[0,2 \pi)$, and $\tilde{\gamma} \in[0,4 \pi)$. The smeared charge distribution

$$
\rho_{\mathrm{D} 7}=\frac{N_{f}}{4 \pi^{3}}
$$

satisfies the property

$$
N_{f}=\int \operatorname{dvol}_{\tilde{S}^{3}} \mathrm{~d} \alpha \rho_{\mathrm{D} 7} .
$$

The generalized embedding (C.33) corresponds to the vanishing of

$$
\begin{aligned}
& f_{1}=\tilde{\psi}-\varphi^{1}-\varphi^{2}-\tilde{\gamma}-\tilde{\varphi}+2 \arg \left(\Gamma_{1}+\Gamma_{2}\right)-2 \alpha+4 \pi n, \\
& f_{2}=\mathrm{e}^{3 \rho}\left|\Gamma_{1}+\Gamma_{2}\right|^{2}-\mathrm{e}^{3 \rho_{\nu}},
\end{aligned}
$$

where $n \in Z$. The Poincaré duals of the smeared D7s again follow from

$$
\Omega=\int \operatorname{dvol}_{\tilde{S}^{3}} \mathrm{~d} \alpha \rho_{\mathrm{D} 7} \delta\left(f_{1}\right) \delta\left(f_{2}\right) \mathrm{d} f_{1} \wedge f_{2} .
$$

Following steps as in the flat space case $\left(\theta^{i}=0\right.$ is a convenient point to evaluate the integral), we find [73]

$$
Q= \begin{cases}0 & \rho<\rho_{\nu}, \\ \frac{3 N_{f}}{4 \pi}\left(1-\mathrm{e}^{3\left(\rho_{\nu}-\rho\right)}\right) & \rho \geq \rho_{\nu} .\end{cases}
$$

where we have written

$$
F_{1}=-Q(\rho)\left(\frac{1}{3} \mathrm{~d} \psi+\mathcal{A}\right)
$$

and used $\mathrm{d} F_{1}=-\Omega$.

We again focus on the massless limit, $\rho_{\nu} \rightarrow-\infty$. Writing the perturbed metric as

$$
\mathrm{d} s_{6}^{2}=\alpha^{\prime}\left\{\mathrm{e}^{2 f}\left[\mathrm{~d} \rho^{2}+\left(\frac{1}{3} \mathrm{~d} \psi+\mathcal{A}\right)^{2}\right]+\mathrm{e}^{2 g} \mathrm{~d} s_{S^{2} \times S^{2}}^{2}\right\},
$$


we find that the equations of motion are solved by (C.18) with the replacement [44]

$$
\frac{g_{\mathrm{s}} N_{f}}{2 \pi} \rightarrow \frac{3 g_{\mathrm{s}} N_{f}}{4 \pi}
$$

The essentially identical solution is a consequence of the common conical nature and Einstein-Sasaki base of $C^{3}$ and the conifold [44]. Similarly, the backreaction of $\overline{\mathrm{D} 3} \mathrm{~s}$ is once again given by (C.30) with the above replacement.

\section{References}

[1] J. M. Maldacena, The large $N$ limit of superconformal field theories and supergravity, Adv.Theor.Math.Phys. 2 (1998) 231-252, [hep-th/9711200].

[2] E. Witten, Anti de Sitter space and holography, Adv.Theor.Math.Phys. 2 (1998) 253-291, [hep-th/9802150].

[3] S. Gubser, I. R. Klebanov, and A. M. Polyakov, Gauge theory correlators from noncritical string theory, Phys.Lett. B428 (1998) 105-114, [hep-th/9802109].

[4] O. Aharony, S. S. Gubser, J. M. Maldacena, H. Ooguri, and Y. Oz, Large N field theories, string theory and gravity, Phys.Rept. 323 (2000) 183-386, [hep-th/9905111].

[5] L. Randall and R. Sundrum, A large mass hierarchy from a small extra dimension, Phys. Rev. Lett. 83 (1999) 3370-3373, [hep-ph/9905221].

[6] H. L. Verlinde, Holography and compactification, Nucl.Phys. B580 (2000) 264-274, [hep-th/9906182].

[7] S. B. Giddings, S. Kachru, and J. Polchinski, Hierarchies from fluxes in string compactifications, Phys.Rev. D66 (2002) 106006, [hep-th/0105097].

[8] D. Baumann, A. Dymarsky, S. Kachru, I. R. Klebanov, and L. McAllister, D3-brane potentials from fluxes in AdS/CFT, JHEP 1006 (2010) 072, [arXiv:1001.5028].

[9] S. Gandhi, L. McAllister, and S. Sjörs, A toolkit for perturbing flux compactifications, JHEP 1112 (2011) 053, [arXiv:1106.0002].

[10] Y. Grossman and M. Neubert, Neutrino masses and mixings in non-factorizable geometry, Phys. Lett. B474 (2000) 361-371, [hep-ph/9912408].

[11] D. Cremades, L. Ibáñez, and F. Marchesano, Computing Yukawa couplings from magnetized extra dimensions, JHEP 0405 (2004) 079, [hep-th/0404229].

[12] B. S. Acharya, F. Benini, and R. Valandro, Warped models in string theory, hep-th/0612192.

[13] A. Karch and E. Katz, Adding flavor to AdS/CFT, JHEP 0206 (2002) 043, [hep-th/0205236].

[14] S. Kachru, D. Simić, and S. P. Trivedi, Stable non-supersymmetric throats in string theory, JHEP 1005 (2010) 067, [arXiv:0905.2970].

[15] A. Dymarsky and S. Kuperstein, Non-supersymmetric conifold, JHEP 1208 (2012) 033, [arXiv:1111.1731].

[16] I. R. Klebanov and M. J. Strassler, Supergravity and a confining gauge theory: Duality cascades and $\chi S B$ resolution of naked singularities, JHEP 0008 (2000) 052, [hep-th/0007191].

[17] S. Kachru, J. Pearson, and H. L. Verlinde, Brane/flux annihilation and the string dual of a nonsupersymmetric field theory, JHEP 0206 (2002) 021, [hep-th/0112197].

[18] O. DeWolfe, S. Kachru, and M. Mulligan, A gravity dual of metastable dynamical supersymmetry breaking, Phys.Rev. D77 (2008) 065011, [arXiv:0801.1520]. 
[19] F. Benini, A. Dymarsky, S. Franco, S. Kachru, D. Simic, and H. Verlinde, Holographic gauge mediation, JHEP 0912 (2009) 031, [arXiv:0903.0619].

[20] P. McGuirk, G. Shiu, and Y. Sumitomo, Holographic gauge mediation via strongly coupled messengers, Phys.Rev. D81 (2010) 026005, [arXiv:0911.0019].

[21] W. Fischler and W. T. Garcia, Hierarchies of susy splittings in holographic gauge mediation, JHEP 1106 (2011) 046, [arXiv: 1104.2078].

[22] P. McGuirk, Hidden-sector current-current correlators in holographic gauge mediation, Phys.Rev. D85 (2012) 045025, [arXiv:1110.5075].

[23] M. Gabella, T. Gherghetta, and J. Giedt, A gravity dual and LHC study of single-sector supersymmetry breaking, Phys.Rev. D76 (2007) 055001, [arXiv:0704.3571].

[24] M. McGarrie and D. C. Thompson, Warped general gauge mediation, Phys.Rev. D82 (2010) 125034, [arXiv:1009.4696].

[25] K. Skenderis and M. Taylor, Holographic realization of gauge mediated supersymmetry breaking, JHEP 1209 (2012) 028, [arXiv: 1205.4677].

[26] R. Argurio, M. Bertolini, L. Di Pietro, F. Porri, and D. Redigolo, Holographic correlators for general gauge mediation, JHEP 1208 (2012) 086, [arXiv:1205.4709].

[27] R. Argurio, M. Bertolini, L. Di Pietro, F. Porri, and D. Redigolo, Exploring holographic general gauge mediation, JHEP 1210 (2012) 179, [arXiv:1208.3615].

[28] M. McGarrie, Holography for general gauge mediation, arXiv:1210.4935.

[29] P. McGuirk, G. Shiu, and Y. Sumitomo, Non-supersymmetric infrared perturbations to the warped deformed conifold, Nucl.Phys. B842 (2011) 383-413, [arXiv:0910.4581].

[30] I. Bena, M. Graña, and N. Halmagyi, On the existence of meta-stable vacua in Klebanov-Strassler, JHEP 1009 (2010) 087, [arXiv:0912.3519].

[31] I. Bena, G. Giecold, M. Graña, and N. Halmagyi, On the inflaton potential from antibranes in warped throats, JHEP 1207 (2012) 140, [arXiv:1011.2626].

[32] I. Bena, G. Giecold, M. Graña, N. Halmagyi, and S. Massai, On metastable vacua and the warped deformed conifold: Analytic results, arXiv:1102.2403.

[33] I. Bena, G. Giecold, M. Graña, N. Halmagyi, and S. Massai, The backreaction of anti-D3 branes on the Klebanov-Strassler geometry, arXiv:1106.6165.

[34] S. Massai, A comment on anti-brane singularities in warped throats, arXiv:1202.3789.

[35] I. Bena, M. Graña, S. Kuperstein, and S. Massai, $\overline{D 3}$ 's - Singular to the bitter end, arXiv:1206.6369.

[36] A. Dymarsky, On gravity dual of a metastable vacuum in Klebanov-Strassler theory, JHEP 1105 (2011) 053, [arXiv: 1102.1734].

[37] J. Blåbäck, U. H. Danielsson, D. Junghans, T. Van Riet, T. Wrase, and M. Zagermann, The problematic backreaction of SUSY-breaking branes, JHEP 1108 (2011) 105, [arXiv:1105.4879].

[38] J. Blåbäck, U. H. Danielsson, D. Junghans, T. Van Riet, T. Wrase, and M. Zagermann, (Anti-)Brane backreaction beyond perturbation theory, JHEP 1202 (2012) 025, [arXiv:1111.2605].

[39] J. Blåbäck, U. H. Danielsson, and T. Van Riet, Resolving anti-brane singularities through time-dependence, arXiv:1202.1132.

[40] I. Bena, D. Junghans, S. Kuperstein, T. Van Riet, T. Wrase, and M. Zagermann, Persistent anti-brane singularities, JHEP 1210 (2012) 078, [arXiv:1205.1798]. 
[41] S. Kachru, R. Kallosh, A. D. Linde, and S. P. Trivedi, De Sitter vacua in string theory, Phys.Rev. D68 (2003) 046005, [hep-th/0301240].

[42] S. Kachru, R. Kallosh, A. D. Linde, J. M. Maldacena, L. P. McAllister, and S. Trivedi, Towards inflation in string theory, JCAP 0310 (2003) 013, [hep-th/0308055].

[43] J. Polchinski, String theory. Vol. 2: Superstring theory and beyond. Cambridge University Press, 1998.

[44] F. Benini, F. Canoura, S. Cremonesi, C. Núñez, and A. V. Ramallo, Unquenched flavors in the Klebanov-Witten model, JHEP 0702 (2007) 090, [hep-th/0612118].

[45] C. Núñez, Á. Paredes, and A. V. Ramallo, Unquenched flavor in the gauge/gravity correspondence, Adv.High Energy Phys. 2010 (2010) 196714, [arXiv: 1002.1088].

[46] P. Brax, G. Mandal, and Y. Oz, Supergravity description of non-BPS branes, Phys.Rev. D63 (2001) 064008, [hep-th/0005242].

[47] B. Zhou and C.-J. Zhu, The complete black brane solutions in D-dimensional coupled gravity system, hep-th/9905146.

[48] F. Bigazzi, A. L. Cotrone, J. Mas, A. Paredes, A. V. Ramallo, and J. Tarrío, D3-D7 quark-gluon plasmas, JHEP 0911 (2009) 117, [arXiv:0909.2865].

[49] F. Bigazzi, A. L. Cotrone, A. Paredes, and A. V. Ramallo, Screening effects on meson masses from holography, JHEP 0905 (2009) 034, [arXiv:0903.4747].

[50] P. Candelas and X. C. de la Ossa, Comments on conifolds, Nucl.Phys. B342 (1990) 246-268.

[51] I. R. Klebanov and E. Witten, Superconformal field theory on three-branes at a Calabi-Yau singularity, Nucl.Phys. B536 (1998) 199-218, [hep-th/9807080].

[52] S. Kuperstein, Meson spectroscopy from holomorphic probes on the warped deformed conifold, JHEP 0503 (2005) 014, [hep-th/0411097].

[53] P. Ouyang, Holomorphic D'7 branes and flavored $\mathcal{N}=1$ gauge theories, Nucl.Phys. B699 (2004) 207-225, [hep-th/0311084].

[54] F. Benini, A chiral cascade via backreacting D7-branes with flux, JHEP 0810 (2008) 051, [arXiv:0710.0374].

[55] H.-Y. Chen, P. Ouyang, and G. Shiu, On supersymmetric D\%-branes in the warped deformed conifold, JHEP 1001 (2010) 028, [arXiv:0807.2428].

[56] I. R. Klebanov and A. A. Tseytlin, Gravity duals of supersymmetric SU $(N) \times \mathrm{SU}(N+M)$ gauge theories, Nucl.Phys. B578 (2000) 123-138, [hep-th/0002159].

[57] R. Minasian and D. Tsimpis, On the geometry of non-trivially embedded branes, Nucl.Phys. B572 (2000) 499-513, [hep-th/9911042].

[58] P. McGuirk, G. Shiu, and F. Ye, Soft branes in supersymmetry-breaking backgrounds, JHEP 1207 (2012) 188, [arXiv:1206.0754].

[59] C. P. Herzog, I. R. Klebanov, and P. Ouyang, Remarks on the warped deformed conifold, hep-th/0108101.

[60] P. G. Cámara, L. Ibáñez, and A. Uranga, Flux-induced SUSY-breaking soft terms on D7-D3 brane systems, Nucl.Phys. B708 (2005) 268-316, [hep-th/0408036].

[61] D. Lüst, S. Reffert, and S. Stieberger, Flux-induced soft supersymmetry breaking in chiral type IIB orientifolds with D3/D\%-branes, Nucl.Phys. B706 (2005) 3-52, [hep-th/0406092].

[62] D. Lüst, S. Reffert, and S. Stieberger, MSSM with soft SUSY breaking terms from D7-branes with 
fluxes, Nucl.Phys. B727 (2005) 264-300, [hep-th/0410074].

[63] D. Lüst, F. Marchesano, L. Martucci, and D. Tsimpis, Generalized non-supersymmetric flux vacua, JHEP 0811 (2008) 021, [arXiv:0807.4540].

[64] M. Kruczenski, D. Mateos, R. C. Myers, and D. J. Winters, Meson spectroscopy in AdS/CFT with flavour, JHEP 0307 (2003) 049, [hep-th/0304032].

[65] F. Marchesano, P. McGuirk, and G. Shiu, Open string wavefunctions in warped compactifications, JHEP 0904 (2009) 095, [arXiv:0812.2247].

[66] F. Marchesano, P. McGuirk, and G. Shiu, Chiral matter wavefunctions in warped compactifications, JHEP 1105 (2011) 090, [arXiv:1012.2759].

[67] S. Cecotti, M. C. Cheng, J. J. Heckman, and C. Vafa, Yukawa couplings in F-theory and non-commutative geometry, arXiv:0910.0477.

[68] F. Marchesano and L. Martucci, Non-perturbative effects on seven-brane yukawa couplings, Phys.Rev.Lett. 104 (2010) 231601, [arXiv:0910.5496].

[69] M. Graña and J. Polchinski, Gauge/gravity duals with holomorphic dilaton, Phys.Rev. D65 (2002) 126005, [hep-th/0106014].

[70] B. A. Burrington, J. T. Liu, L. A. Pando Zayas, and D. Vaman, Holographic duals of flavored $\mathcal{N}=1$ super Yang-Mills: Beyond the probe approximation, JHEP 0502 (2005) 022, [hep-th/0406207].

[71] M. Ihl, A. Kundu, and S. Kundu, Back-reaction of non-supersymmetric probes: Phase transition and stability, JHEP 1212 (2012) 070, [arXiv:1208.2663].

[72] F. Bigazzi, A. L. Cotrone, and A. Paredes, Klebanov-Witten theory with massive dynamical flavors, JHEP 0809 (2008) 048, [arXiv:0807.0298].

[73] F. Bigazzi, A. L. Cotrone, A. Paredes, and A. Ramallo, Non chiral dynamical flavors and screening on the conifold, Fortsch.Phys. 57 (2009) 514-520, [arXiv:0810.5220]. 